\title{
Langzeitverlauf einer sekundären Rechtsherzbelastung nach perkutaner Mitralklappenreparatur mittels MitraClip@
}

\author{
INAUGURAL-DISSERTATION \\ zur Erlangung des Doktorgrades \\ der Medizinischen Fakultät \\ der Georg-August-Universität zu Göttingen
}

\author{
vorgelegt von \\ Bo Eric Beuthner
}

aus

Braunschweig

Göttingen 2017 
Dekan:

Referentin:

Ko-Referent:

Drittreferent:

Tag der mündlichen Prüfung:
Prof. Dr. rer. nat. Heyo Klaus Kroemer

PD Dr. med. Miriam Puls

Prof. Dr. med. Theodoros Tirilomis

Prof. Dr. med. Matthias Sigler

13.03.2018 
Hiermit erkläre ich, die Dissertation mit dem Titel „Langzeitverlauf einer sekundären Rechtsherzbelastung nach perkutaner Mitralklappenreparatur mittels MitraClip@" eigenständig angefertigt und keine anderen als die von mir angegebenen Quellen und Hilfsmittel verwendet zu haben.

Göttingen, den 


\section{Inhaltsverzeichnis}

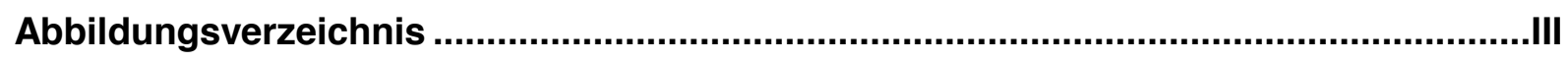

Tabellenverzeichnis ............................................................................................... IV

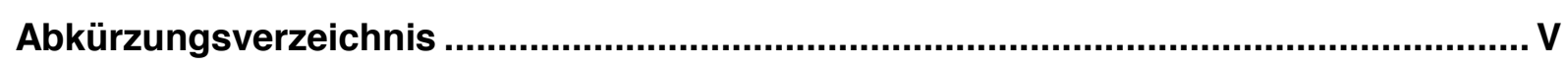

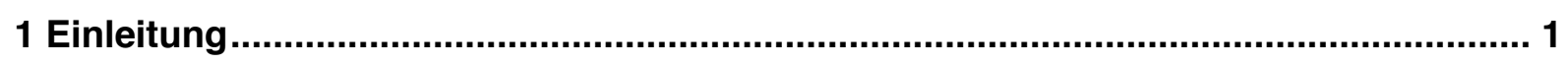

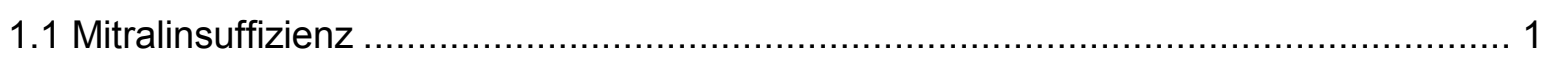

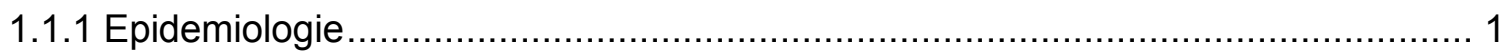

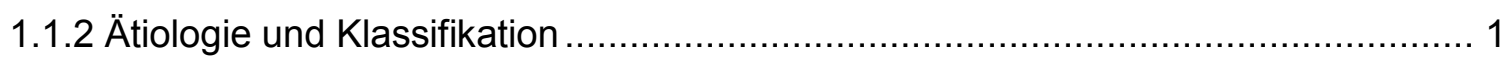

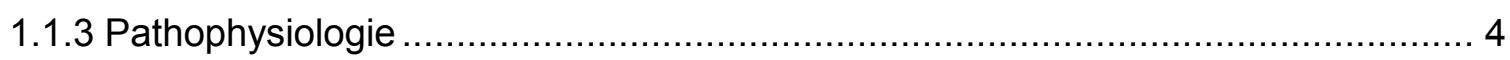

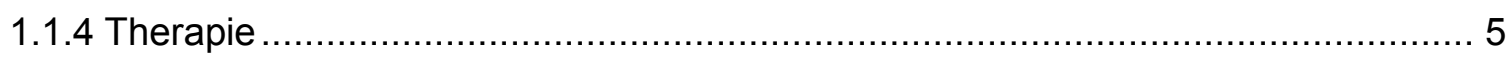

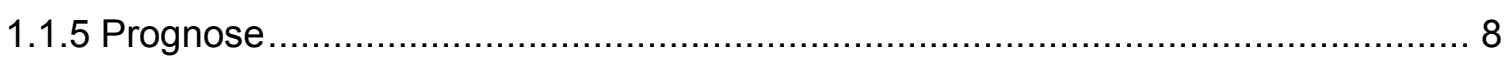

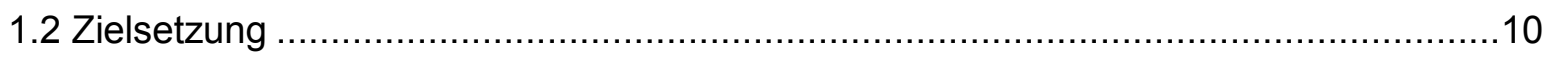

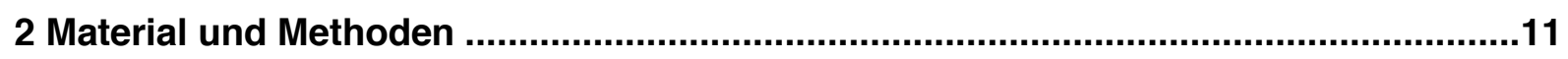

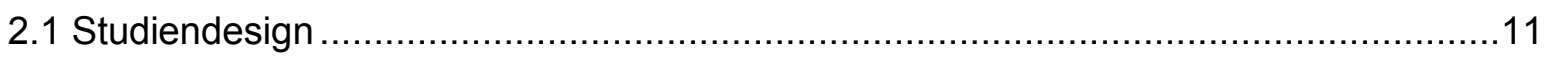

2.2 Präinterventionelle Diagnostik und Patientenselektion ...................................... 11

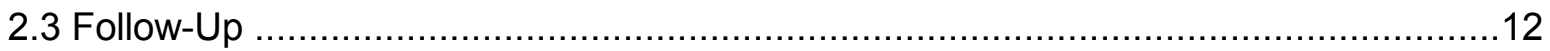

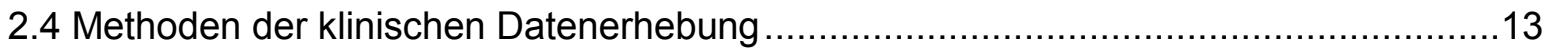

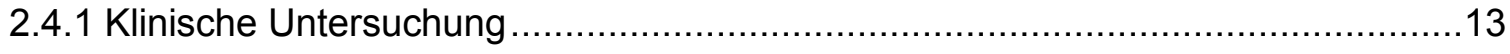

2.4.2 New York Heart Association (NYHA) Klassifikation ......................................13

2.4.3 Sechs-Minuten-Gehtest (6MWT) .......................................................... 13

2.4.4 Minnesota Living with Heart Failure Questionnaire (MLHFQ) .............................14

2.4.5 Echokardiographische Diagnostik der Rechtsherzinsuffizienz ........................... 14

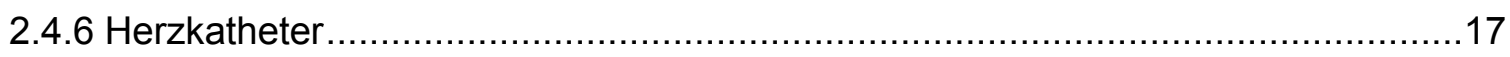

2.4.7 European System for Cardiac Operative Risk Evaluation (EuroSCORE) ..............17

2.4.8 Society of Thoracic Surgeons (STS) Score .............................................. 17

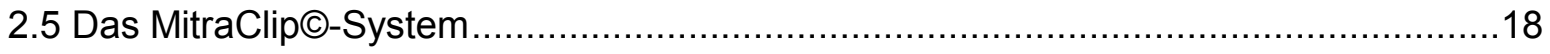

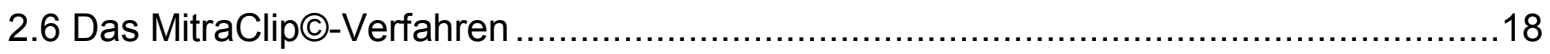

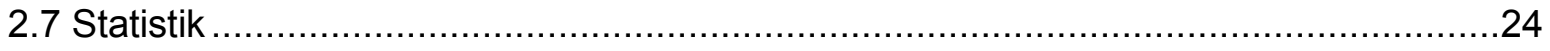

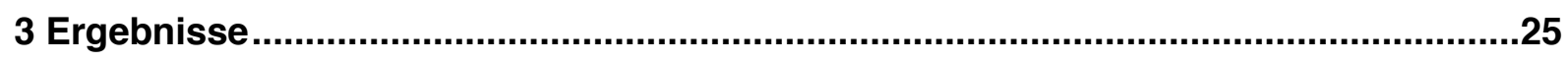

3.1 Demographische und klinische Baseline Charakteristika .....................................25

3.2 Klinischer Erfolg der MitraClip@-Implantation ..................................................

3.3 Veränderungen der Rechtsherzparameter vor Klinikentlassung .............................27

3.4 Veränderungen der Rechtsherzparameter nach 12 Monaten ....................................28

3.5 Langzeitüberleben nach MitraClip@-Implantation bei Patienten mit sekundärer

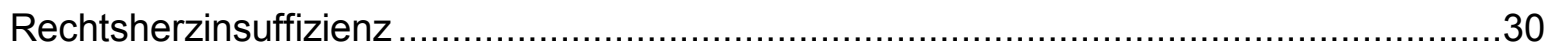


3.6 Langzeitüberleben in Abhängigkeit vom präinterventionellen rechtsventrikulären systolischen Druck.

3.7 Langzeitüberleben in Abhängigkeit von der präinterventionellen rechtsventrikulären Kontraktilität (abgeschätzt durch TAPSE) vor dem Eingriff.

3.8 Langzeitüberleben in Abhängigkeit von der Veränderung der rechtsventrikulären Kontraktilität (abgeschätzt durch TAPSE) nach dem Eingriff.

3.9 Langzeitüberleben in Abhängigkeit von der Veränderung des rechtsventrikulären Druckes nach dem Eingriff .34

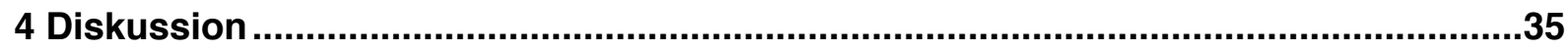

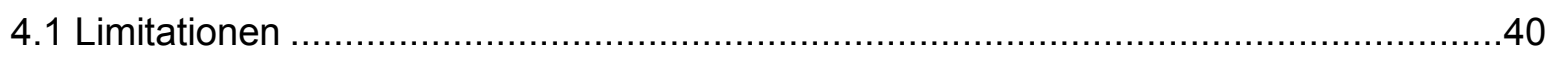

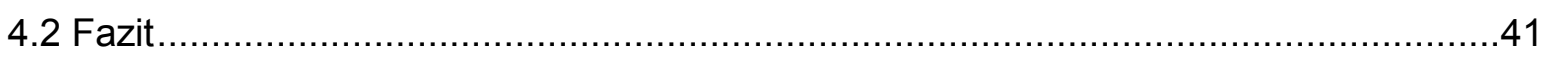

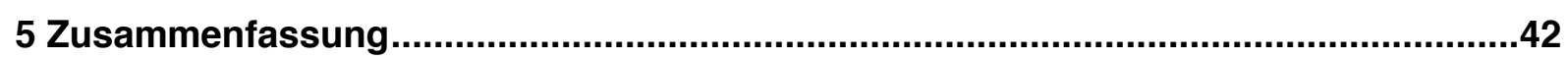

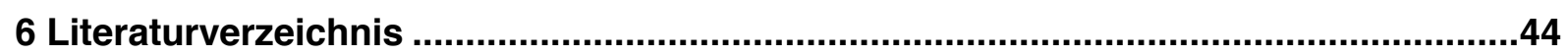

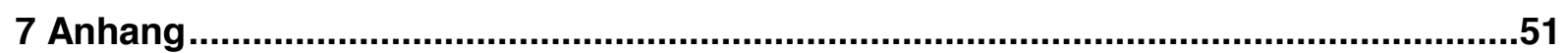

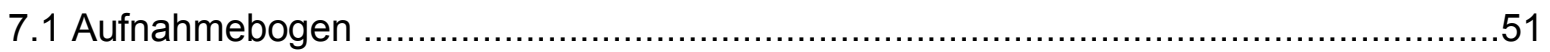

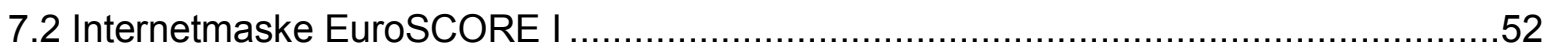

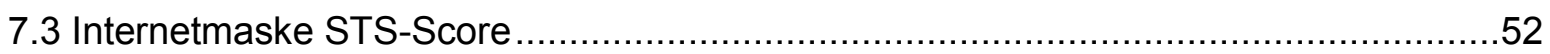

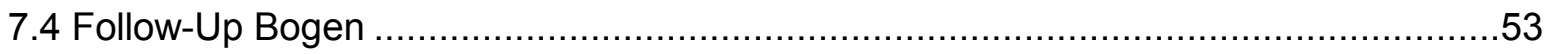

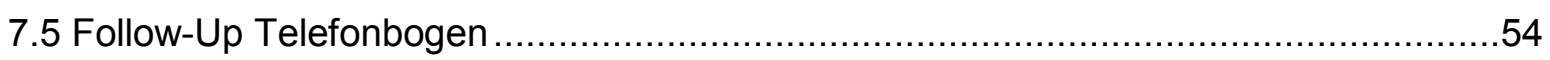




\section{Abbildungsverzeichnis}

Abbildung 1: Anatomie der Mitralklappe

Abbildung 2: Ursachen der sekundären Mitralinsuffizienz, links: "Tenting der Mitralsegel, rechts: Maladaption der Mitralsegel

Abbildung 3: Naht der Mitralklappensegel nach Alfieri

Abbildung 4: Leitlinien zur Therapie der hochgradigen sekundären Mitralinsuffizienz 7

Abbildung 5: Kaplan-Meier-Analyse des Überlebens nach medikamentöser und chirurgischer

Therapie von Patienten mit schwerer Mitralinsuffizienz und pulmonal-arterieller

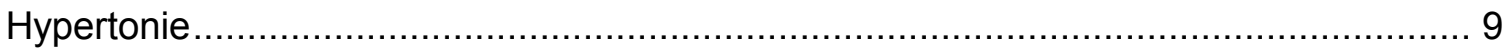

Abbildung 6: Echokardiographische Bestimmung der TAPSE im M-Mode.........................16

Abbildung 7: Ausmessung der rechtsventrikulären Dimensionen im 4-Kammerblick ...........16

Abbildung 8: Das MitraClip@-System..................................................................... 18

Abbildung 9: Zwei mögliche Anordnungen im Herzkatheterlabor .......................................19

Abbildung 10: Darstellung und Evaluation der hochgradigen sekundären MI in der zweidimensionalen Echokardiographie zu Prozedurbeginn

Abbildung 11: Fluoroskopische Darstellung der Punktion des interatrialen Septums sowie des Vorbringens des auf den steuerbaren Führungskatheters montierten Clips in das linke Atrium

Abbildung 12: Linksatriale echokardiographische Ausrichtung des MitraClips@ unter TEEKontrolle.

Abbildung 13: Schließen des Clips unter echokardiographischer Sicht, während beide Segel auf den Clip-Armen aufliegen ....

Abbildung 14: Minimale residuale $\mathrm{MI}$ in der biplanen TEE-Darstellung nach Clip-Platzierung

Abbildung 15: Mitralklappe mit 2 Öffnungen in der 3D-TEE nach Clip-Implantation .23

Abbildung 16: Entwicklung rechtsventrikulärer Parameter zum Entlassungszeitpunkt sowie nach 12 Monaten, dargestellt in box and whiskers-Diagrammen ...............................29

Abbildung 17: Kaplan-Meier-Überlebenskurve der Gesamtkohorte .30

Abbildung 18: Kaplan-Meier-Überlebenskurve in Abhängigkeit vom rechtsventrikulären systolischen Druck

Abbildung 19: Kaplan-Meier-Überlebenskurve in Abhängigkeit von der präinterventionellen rechtsventrikulären Kontraktilität.

Abbildung 20: Kaplan-Meier-Überlebenskurve in Abhängigkeit von der Veränderung der rechtsventrikulären Kontraktilität nach dem Eingriff

Abbildung 21: Kaplan-Meier-Überlebenskurve in Abhängigkeit von der Veränderung des rechtsventrikulären Druckes nach dem Eingriff. 


\section{Tabellenverzeichnis}

Tabelle 1: Basisdemographische Charakteristika des Gesamtkollektivs $(n=70)$. .26 


\section{Abkürzungsverzeichnis}

AHA

ACC

ASS

ECLIA

EKG

et al.

GFR

EuroSCORE

INR

LVEDV

LVESV

MLHFQ

NT-pro BNP

NYHA

PAPSyst

PCWP

$\mathrm{PH}$

RVOT

STS-Score

TAPSE

TEE

TR

TTE

6MVT
American Heart Association

American College of Cardiology

Acetylsalicylsäure

Electro-Chemiluminescence Immunoassay

Elektrokardiogramm

et alii

Glomeruläre Filtrationsrate

European System for Cardiac Operative Risk Evaluation

International Normalized Ratio

Linksventrikuläres enddiastolisches Volumen

Linksventrikuläres endsystolisches Volumen

Minnesota Living with Heart Failure Questionnaire

$\mathrm{N}$-terminal prohormone of Brain Natriuretic Peptide

New York Heart Association

Pulmonary Artery Systolic Pressure

Pulmonary Capillary Wedge Pressure

Pulmonary Hypertension

Right Ventricular Outflow Tract

Society of Thoracic Surgeons- Score

Tricuspid Annular Plane Systolic Excursion

Transösophageale Echokardiographie

Trikuspidale Regurgitation

Transthorakale Echokardiographie

Sechs-Minuten-Gehtest 


\section{Einleitung}

\subsection{Mitralinsuffizienz}

Die Mitralklappe befindet sich zwischen dem linken Vorhof und dem linken Ventrikel. Sie besteht aus zwei Klappensegeln, die während der Diastole geöffnet sind, um einen Blutfluss von linkem Vorhof in den linken Ventrikel zu ermöglichen. Während der Systole liegen die beiden Klappensegel physiologischerweise aneinander an, die Klappe ist geschlossen. Die Schlussunfähigkeit dieser Herzklappe wird als Mitralinsuffizienz bezeichnet. Dabei kommt es zu einer Regurgitation in den linken Vorhof.

\subsubsection{Epidemiologie}

Die Mitralklappeninsuffizienz ist nach der Aortenstenose der zweithäufigste Herzklappenfehler in Europa (lung 2003). Die Deutsche Gesellschaft für Kardiologie schätzt, dass alleine in Deutschland 800000 bis 1 Million Menschen an einer therapierelevanten Mitralinsuffizienz leiden (Nickenig et al. 2013). Das durchschnittliche Alter der Betroffenen ist aufgrund der Fortschritte in der Therapie rheumatischer Erkrankungen deutlich angestiegen und liegt derzeit bei 65 Jahren (Erbel et al. 2009). Generell wird in der Gesamtbevölkerung von einer Prävalenz der Erkrankung von 1-2\% ausgegangen, die in Abhängigkeit vom Alter auf bis zu $10 \%$ bei über 75-jährigen Patienten steigt (Nkomo et al. 2006).

\subsection{2 Ätiologie und Klassifikation}

Die ordnungsgemäße Funktion der Mitralklappe setzt das erfolgreiche Zusammenspiel zahlreicher anatomischer Strukturen wie Mitralklappensegel, Anulus, Chordae tendineae, Papillarmuskeln sowie linkem Vorhof und linkem Ventrikel voraus. Kenntnis dieser Strukturen und ihres Zusammenspiels ist notwendig für ein Verständnis der Entstehung und Einteilung der Mitralinsuffizienz (siehe Abbildung 1). 


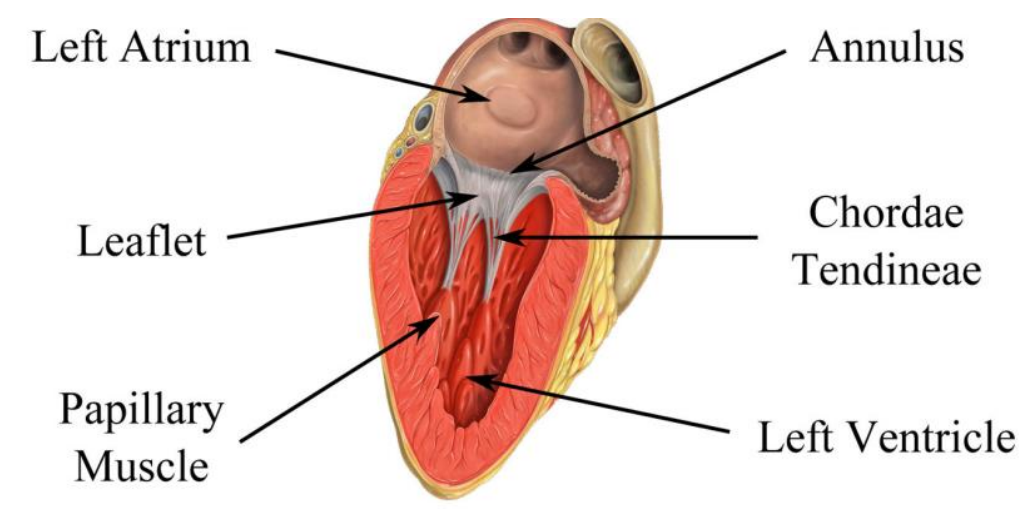

Abbildung 1: Anatomie der Mitralklappe (aus Tenenholtz et al. 2010)

Sowohl vorderes als auch hinteres Segel der Mitralklappe werden während der Systole durch die an den Papillarmuskeln befestigten Sehnenfäden (Chordae tendineae) am Zurückschlagen in den linken Vorhof gehindert. Dabei ist zu beachten, dass die Papillarmuskeln nicht durch aktive Kontraktion für die Klappenfunktion zuständig sind. Diese wird durch Bewegung der Ventilebene entsprechend der Druckverhältnisse im Herzen erreicht (Schmidt et al. 2010).

Die Einteilung der Mitralinsuffizienz erfolgt nach zeitlichem Verlauf, Mechanismus und Ursache (Enriquez-Sarano et al. 2009). Dabei sind Mechanismus und Ursache nicht synonym zu verstehen. Nach zeitlichem Verlauf wird die Mitralinsuffizienz in akut und chronisch unterteilt. Häufigste Ursachen einer akuten Mitralinsuffizienz sind schnell destruierende Ereignisse wie die Sehnenfadenruptur (zum Beispiel infolge einer Endokarditis) oder der Papillarmuskelabriss infolge eines Myokardinfarktes (Crawford 1986). Das plötzliche hohe Regurgitationsvolumen, das während der Systole zurück in den linken Vorhof fließt, führt zu einer plötzlichen Volumenüberladung des unvorbereiteten linken Ventrikels mit konsekutivem Lungenödem (Marak et al. 2013). Daher handelt es sich bei der akuten Mitralinsuffizienz um einen Notfall, der gemäß den gültigen Leitlinien chirurgisch versorgt werden sollte (Vahanian et al. 2012).

Die vorliegende Arbeit beschäftigt sich vor allem mit der chronischen Mitralinsuffizienz. Hier ist eine weitere Klassifikation nach Mechanismus und Ursache besonders relevant, da diese unterschiedlich therapiert werden (Carpentier 1983). So wird die chronische Mitralinsuffizienz in primäre und sekundäre Mitralinsuffizienz unterteilt, wobei die primäre Ätiologie der Mitralinsuffizienz mit ungefähr 60\% überwiegt (lung 2003). Entscheidend für die Zuordnung $\mathrm{zu}$ einem der beiden Mechanismen ist die Klappenmorphologie. Die primäre Mitralinsuffizienz umfasst dabei alle Formen der Mitralinsuffizienz, denen eine Pathologie der Klappensegel, Sehnenfäden oder Papillarmuskeln zugrunde liegt. Hingegen entwickelt sich die sekundäre Mitralinsuffizienz meistens infolge einer Geometrieänderung des 
Klappenhalteapparates aufgrund von Dilatation und Remodelling des linken Ventrikels bzw. des Mitralklappenanulus und daraus resultierender Maladaptation der Klappensegel (Enriquez-Sarano et al. 2009).

Die Ursachen der chronischen Mitralinsuffizienz werden weiterhin in ischämische und nichtischämische Ursachen eingeteilt. Unter ischämischen Ursachen versteht man das Remodelling des linken Ventrikels auf der Grundlage einer koronaren Herzkrankheit. Es kommt zu einem Zug am Mitralklappenapparat in den Ventrikel (tethering), wodurch anteriores und posteriores Segel auseinanderrücken und das vollständige Schließen der Mitralklappe unmöglich machen (Kono et al. 1992; Enriquez-Sarano et al. 2009) (vgl. auch Abb. 2; aus Puls und Schillinger 2016).
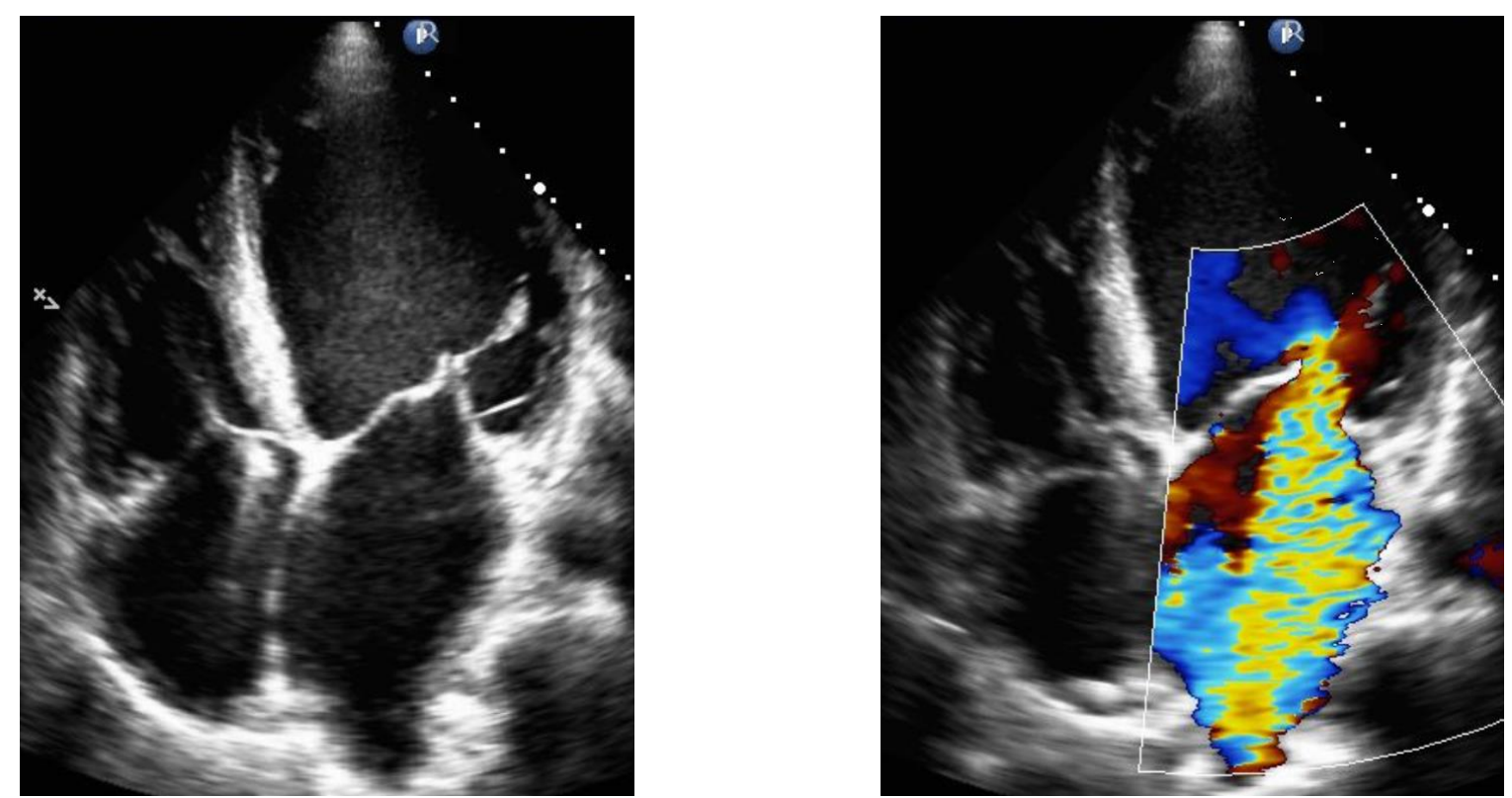

Abbildung 2: links: stark dilatierter linker Ventrikel mit Papillarmuskelverlagerung und konsekutivem Zug an den Sehnenfäden, sodass der Klappenschluss nicht mehr auf AVKlappenebene stattfindet, sondern in den Ventrikel verlagert ist (Tenting der Mitralsegel) rechts: sekundäre Mitralinsuffizienz infolge Maladaptation der Mitralsegel in dieser Position (aus Puls und Schillinger 2016)

Nicht-ischämische Ursachen umfassen beispielsweise die Degeneration der Mitralklappe infolge einer Endokarditis oder einer rheumatischen Erkrankung. 


\subsubsection{Pathophysiologie}

Die Schlussunfähigkeit der Mitralklappe während der Systole führt zu einem Rückstrom von Blut in den linken Vorhof. Diese Steigerung der Vorlast bewirkt eine kompensatorische Dilatation von Vorhof und Ventrikel. Die dauerhafte Volumenbelastung des linken Ventrikels führt langfristig zu einer Schädigung der Kontraktilität des Myokards durch Verlängerung der Myozyten und Verlust myofibrillären Inhaltes (Spinale et al. 1993; Aeschlimann und Siegenthaler 2005). Die Vergrößerung des Vorhofes wird als eine Ursache von Vorhofflimmern und damit auch der Entstehung von Vorhofthromben angesehen (January et al. 2014).

Darüber hinaus geht der chronische Rückstau von Blut in den linken Vorhof und somit in die Lungenvenen mit einem erhöhten linksatrialen Druck einher, der passiv und durch reaktive Vasokonstriktion zu einer konsekutiven sekundären pulmonalen Hypertonie führt (Delgado et al. 2005; Erdmann 2011). Aus der chronischen pulmonal-venösen Stauung resultiert eine endotheliale Dysfunktion mit neurohumoraler Aktivierung (Hassoun et al. 2009; Morrell et al. 2009). Im Rahmen dieser Prozesse kommt es zu einem pulmonal-vaskulären Remodelling aller Wandschichten, das vor allem pulmonale Arteriolen und Arterien betrifft (Delgado et al. 2005). Auf diese Weise entsteht aus der ursprünglich pulmonal-venösen Stauung eine sekundäre pulmonal-arterielle Hypertonie. Das Ausmaß der reaktiven pulmonalen Vasokonstriktion sowie des begleitenden pulmonal-vaskulären Remodellings ist individuell sehr unterschiedlich. Neben dem Ausmaß der Mitralinsuffizienz ist es auch abhängig von weiteren Faktoren wie zum Beispiel einer schwer eingeschränkten linksventrikulären Ejektionsfraktion (Carabello 2013; Hardegree et al. 2013; Le Tourneau et al. 2013). Die pulmonal-vaskuläre Hypertonie entspricht einer erhöhten Nachlast für das rechte Herz und führt somit zu einer Rechtsherzbelastung. Resultiert diese anfänglich noch in einer physiologischen, adaptiven Rechtsherzhypertrophie, kommt es mit weiterem Bestehen der pathologischen Druckverhältnisse zu einer maladaptiven Rechtsherzhypertrophie, die langfristig zu einer systolischen Dysfunktion mit Abnahme des Herzzeitvolumens und somit zu einer manifesten Rechtsherzinsuffizienz führt (Erdmann 2011).

Zum einen ist das Vorliegen einer pulmonal-arteriellen Hypertonie bei Patienten mit primärer Mitralinsuffizienz von Bedeutung, da sie als unabhängiger Prädiktor für die Mortalität identifiziert werden konnte (Le Tourneau et al. 2010; Barbieri et al. 2011). Zum anderen ist für die chirurgische Mitralklappenreparatur aus früheren Arbeiten bekannt, dass bei Patienten mit eingeschränkter linksventrikulärer Funktion und pulmonal-venöser Hypertonie die rechtsventrikuläre Funktion prognostische Bedeutung hat (Bonow et al. 2006; Chrustowicz et al. 2010). 


\subsubsection{Therapie}

Die Therapieoptionen der Mitralinsuffizienz umfassen drei Möglichkeiten.

Die erste Säule ist die chirurgische Korrektur, die bei der schweren primären Mitralinsuffizienz gemäß aktuellen Leitlinien therapeutischer Goldstandard ist und eine Evidenzgrad 1C-Empfehlung hat (Vahanian et al. 2012).

Bei der sekundären Mitralinsuffizienz hingegen ist die Therapie weniger eindeutig, da auch die zur Insuffizienz führende Primärerkrankung in der Therapie der Mitralinsuffizienz berücksichtigt werden muss. So gibt es für die Chirurgie dort nur bei Patienten mit schwerer sekundärer Mitralinsuffizienz, die aber noch eine erhaltene Pumpfunktion besitzen und bei denen primär eine Bypass-Operation geplant ist, eine Klasse 1-Empfehlung.

Grundsätzlich bestehen die chirurgischen Behandlungsmöglichkeiten einer Mitralinsuffizienz aus Klappenreparatur und Klappenersatz. Retrospektive Studien haben lange auf einen Überlebensvorteil für Patienten mit Klappenreparatur hingewiesen (Suri et al. 2006; Jokinen et al. 2007; Shuhaiber und Anderson 2007; Chikwe et al. 2011). Eine erste prospektiv randomisierte Studie an 251 Patienten mit sekundärer Mitralinsuffizienz ischämischer Genese konnte 2014 keinen statistisch signifikanten Überlebensvorteil für eine der beiden Therapieoptionen aufzeigen (1-Jahres-Mortalität 14,3\% nach Reparatur und 17,6\% nach Klappenersatz, $p=0,45)$. Jedoch legen die Ergebnisse der Studie nahe, dass ein Klappenersatz eine längerfristige und effektivere Reduktion der Mitralinsuffizienz bewirkt (Prävalenz einer mittelgradigen oder schweren residualen MI 32,6\% nach Klappenreparatur und 2,3\% nach Klappenersatz, $p<0,001$ ). Gleichzeitig zeigt die neue Studie einen Trend hin zu einem erhöhten Mortalitätsrisiko, insbesondere innerhalb der ersten 30 postoperativen Tage bei Klappenersatz (4,0\% vs. 1,6\%) (Acker et al. 2014).

Eine zweite Säule in der Therapie der Mitralinsuffizienz stellt mittlerweile das MitraClip@Verfahren dar, welches bereits Eingang in die europäischen Leitlinien (Vahanian et al. 2012) gefunden hat. Es beruht auf dem chirurgischen Konzept der edge-to-edge Reparatur, die 1992 von dem italienischen Herzchirurgen Ottavio Alfieri entwickelt wurde (Alfieri et al. 2004). Dabei werden die jeweiligen medialen Segmente von anteriorem und posteriorem Mitralklappensegel mittels fortlaufender Naht zusammengenäht. Die Länge der Naht ist variabel, nutzt in der Regel jedoch die gesamte Länge der medialen Segmente (Maisano et al. 2000; Glower 2012) (siehe Abbildung 3). 


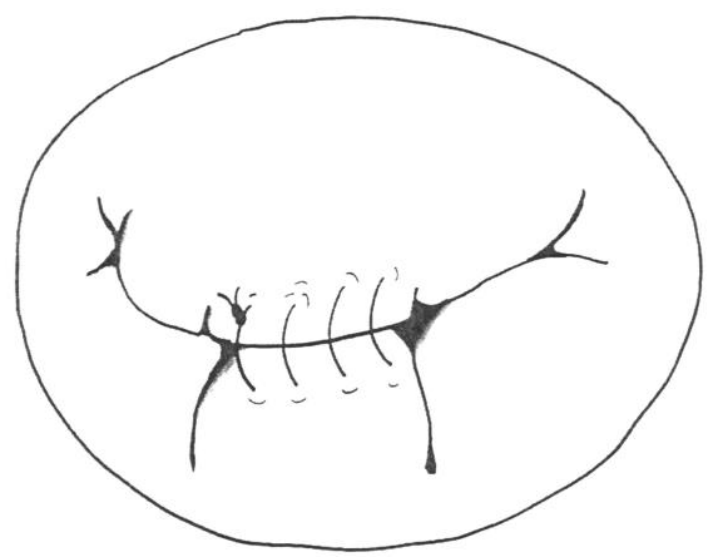

Abbildung 3: Fortlaufende Naht zwischen den medialen Segmenten von anteriorem und posteriorem Mitralklappensegel nach Alfieri (aus Maisano et al. 2000)

Durchführbarkeit, Effektivität und Sicherheit der kathetergestützten Mitralklappenreparatur mittels MitraClip@-System konnten 2009 in der sogenannten EVEREST-I (Endovascular Valve Edge-to-Edge Repair Study)-Studie gezeigt werden (Feldman et al. 2009).

In der prospektiv randomisierten EVEREST-II-Studie wurde 2011 das MitraClip@-Verfahren mit der klassischen Mitralklappenchirurgie verglichen. 279 prinzipiell operable Patienten wurden im Verhältnis 2:1 in die MitraClip@- bzw. die Operationsgruppe randomisiert. Dabei konnte gezeigt werden, dass durch chirurgische Korrektur eine effektivere Reduktion der Mitralinsuffizienz erreicht werden konnte als durch die perkutane Mitralklappenreparatur: Innerhalb des ersten Jahres nach dem Eingriff mussten sich 20\% der interventionell behandelten gegenüber $2 \%$ der operierten Patienten aufgrund einer relevanten residualen Mitralinsuffizienz einem operativen Zweiteingriff unterziehen $(p<0,0001)$. Gleichzeitig wies das MitraClip@-Verfahren eine deutlich niedrigere Komplikationsrate auf. Dieser Unterschied beruhte dabei hauptsächlich auf der geringeren Inzidenz an Blutungsereignissen mit Transfusionsindikation. Während die Komplikationsrate bei dem MitraClip@-Verfahren innerhalb der ersten 30 Tage nach Intervention 15\% betrug, lag sie in der chirurgischen Gruppe bei $48 \%$. Die Mortalität war in beiden Gruppen (sowohl im kurzfristigen wie auch im langfristigen Verlauf bis zu 4 Jahre nach dem Eingriff) nicht unterschiedlich. Die EVERESTII-Studie konnte auch die grundsätzliche Effektivität des Verfahrens bestätigen (Feldman et al. 2011a; Boekstegers et al. 2013): Immerhin 80\% der mittels MitraClip@ behandelten Patienten zeigten gute funktionelle Ergebnisse.

Spätere klinische Studien konnten zudem einen klinischen Nutzen der perkutanen Mitralklappenreparatur bei älteren Patienten (> 75 Jahre) und bei schwerster Herzinsuffizienz nachweisen (Auricchio et al. 2011; Franzen et al. 2011; Maisano et al. 2013; Schillinger et al. 2013). Weiterhin liegen inzwischen Registerdaten von insgesamt fast 2000 mittels MitraClip@-Implantation behandelten Patienten vor (Maisano et al. 2013; Nickenig et al. 
2014; Puls et al. 2016). Diese Registerdaten konnten das geringe periinterventionelle Risiko der MitraClip@-Implantation bestätigen. Darüber hinaus zeigte sich eine Zunahme der prozeduralen Erfolgsrate (gemessen am Grad der residualen Mitralinsuffizienz) in chronologischer Reihenfolge der Publikationen von 77\% in EVEREST-II (Feldman et al. 2011b) auf aktuell 91\% (Maisano et al. 2013) bis 97\% (Puls et al. 2016), was Lernkurve und zunehmende Erfahrung mit diesem technisch anspruchsvollen Verfahren dokumentiert. Von besonderer Bedeutung ist außerdem, dass alle Register eine signifikante Verbesserung der Herzinsuffizienzsymptomatik und Lebensqulität der behandelten Patienten nach dem Eingriff zeigen konnten.

Den aktuellen europäischen Leitlinien (Vahanian et al. 2012) sowie einem Konsensuspapier der Deutschen Gesellschaft für Kardiologie und der Deutschen Gesellschaft für ThoraxHerz-und Gefäßchirurgie (Nickenig et al. 2013) (vgl. Abb. 4) folgend soll die MitraClip@Implantation insbesondere in Erwägung gezogen werden bei Patienten mit schwerer sekundärer MI und hohem operativen Mortalitätsrisiko.

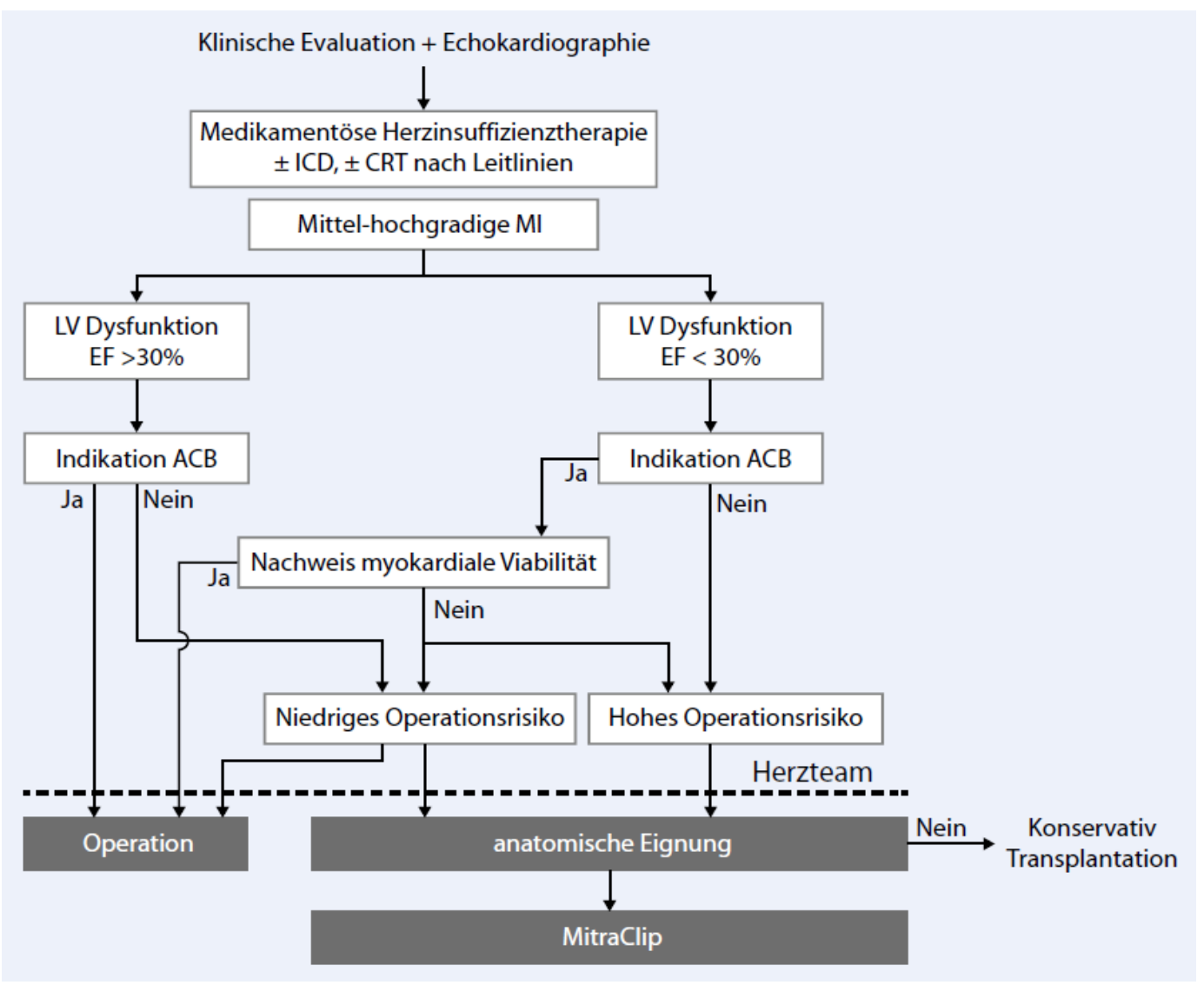

Abbildung 4: Therapie der hochgradigen sekundären Mitralinsuffizienz; aus: Konsensuspapier der Deutschen Gesellschaft für Kardiologie und der Deutschen Gesellschaft für Thorax-Herz-und Gefäßchirurgie (aus Nickenig et al. 2013) 
Aktuell werden weltweit vier randomisierte MitraClip@-Studien durchgeführt. Im Rahmen der COAPT (Cardiovascular Outcomes Assessment of the MitraClip Percutaneous Therapy)Studie in den USA sowie der RESHAPE-HF (Randomized Study of the MitraClip Device in Heart Failure Patients With Clinically Significant Functional Mitral Regurgitation)-Studie in Europa werden herzinsuffiziente Patienten mit sekundärer Mitralinsuffizienz nach Randomisierung einer MitraClip@-Implantation oder einer alleinigen optimalen medikamentösen Herzinsuffizienztherapie zugeführt. Zwei weitere randomisierte Studien vergleichen chirurgische Korrektur und MitraClip@-Implantation. Die MATTERHORN (Mitral vAlve reconsTrucTion for advancEd insufficiency of functional or iscHemic ORigiN)-Studie vergleicht die chirurgische Korrektur mit der MitraClip@-Implantation bei Patienten mit sekundärer Mitralinsuffizienz. Im Gegensatz zur EVEREST-II-Studie werden hier Patienten verglichen, die eine reduzierte linksventrikuläre Ejektionsfraktion und damit ein erhöhtes operatives Risiko aufweisen. Die HiRiDe (High and Intermediate Risk Degenerative Mitral Regurgitation Treatment)-Studie wiederum vergleicht die chirurgische Korrektur der Mitralinsuffizienz mit der MitraClip@-Implantation bei Patienten mit primärer Mitralinsuffizienz. Hier werden ebenfalls Patienten mit mittlerem bis hohem operativem Risiko untersucht (Maisano 2015).

Dritte Säule in der Therapie der Mitralinsuffizienz ist die medikamentöse Therapie. Gemäß den gültigen Leitlinien entspricht sie der Medikation bei Herzinsuffizienz. Jedoch sollte sie bei Patienten mit primärer Mitralklappeninsuffizienz nur dann in Betracht gezogen werden, wenn der Patient zusätzlich eine Herzinsuffizienz entwickelt hat und einer chirurgischen Therapie nicht zugeführt werden kann, beziehungsweise Restsymptome nach chirurgischer Mitralklappenreparatur zeigt. Liegt eine sekundäre Mitralinsuffizienz vor, ist eine optimale medikamentöse Therapie unerlässlich (McMurray et al. 2012; Vahanian et al. 2012).

\subsubsection{Prognose}

Die Prognose der chronischen Mitralinsuffizienz variiert stark in Abhängigkeit von ihrer Ätiologie. Generell geht man bei der sekundären Mitralinsuffizienz von einer schlechteren Prognose als bei der primären Mitralinsuffizienz aus (Grigioni et al. 2001), da hier auch die zur Mitralinsuffizienz führende Grunderkrankung die Prognose beeinflusst und limitiert (Vahanian et al. 2012).

Eine vergleichsweise bessere Prognose haben Patienten mit chronischer primärer Mitralinsuffizienz, die darüber hinaus deutlich von einer alleinigen chirurgischen Mitralklappenreparatur profitieren (Enriquez-Sarano et al. 2009). 
Leiden diese Patienten jedoch zusätzlich präoperativ oder postoperativ an einer pulmonalen Hypertonie, konnten große chirurgische Kohorten zeigen, dass dies einen negativen Einfluss auf Mortalität und Morbidität nach chirurgischer Mitralklappenreparatur hat (Barbieri et al. 2011; Murashita et al. 2015) (siehe Abbildung 5).

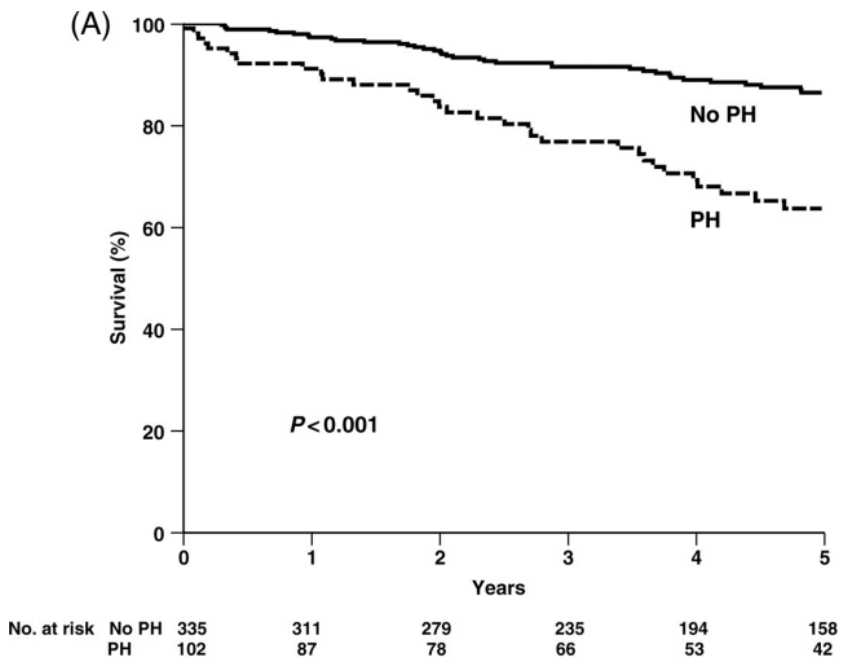

Abbildung 5: Kaplan-Meier-Analyse des Überlebens nach medikamentöser und chirurgischer Therapie von Patienten mit schwerer Mitralinsuffizienz und pulmonal-arterieller Hypertonie, definiert als PAPsyst $>50 \mathrm{mmHg}$ (aus Barbieri et al. 2011)

Wenn sich diese Patienten einer Revaskularisierungsmaßnahme oder einer Herzklappenoperation unterziehen, ist die pulmonale Hypertonie zudem indirekt von prognostischer Bedeutung, da durch die extrakorporale Zirkulation an der Herz-LungenMaschine massiv Zytokine ausgeschüttet werden (Baehner et al. 2012). Auf diese Weise kann eine pulmonale Hypertonie verschlechtert werden oder auch erst ausgelöst werden. Deshalb hat die pulmonale Hypertonie Einfluss auf weitere Behandlungsoptionen der Patienten und ist von prognostischer Relevanz.

Ebenso konnte für eine eingeschränkte rechtsventrikuläre Funktion ein negativer Einfluss auf Mortalität und Morbidität nach isolierter chirurgischer Mitralklappenreparatur nachgewiesen werden (Gallego-Delgado et al. 2012; Ye et al. 2014). So konnten Gallego-Delgado et al. 2012 in einer 109 Patienten umfassenden Studie zeigen, dass das Überleben nach chirurgischer Mitralklappenreparatur bei einem TAPSE-Wert $\leq 16 \mathrm{~mm}$ auf durchschnittlich 453 Tage im Gegensatz zu 705 Tagen eingeschränkt war $(p=0,001)$ (Gallego-Delgado et al. 2012). 
Das Ausmaß der Beeinflussung der Rechtsherzinsuffizienz durch die chirurgische Korrektur des Mitralklappenvitiums ist in der Literatur allerdings inkonstant (Ghoreishi et al. 2011; Goldstone et al. 2011).

Das Vorliegen einer klinischen und/ oder echokardiographischen Rechtsherzinsuffizienz konnte in mehreren Studien als Prädiktor erhöhter Mortalität nach MitraClip@-Implantation identifiziert werden (Neuss et al. 2013; Puls et al. 2014a).

Neuss et al untersuchten 2013157 Patienten, die bei TAPSE-Werten <15mm ein signifikant verkürztes Überleben nach Intervention hatten. Auf dieser Grundlage entwickelten sie einen Algorithmus, der unter anderem TAPSE-Werte kleiner 15mm nutzt, um von einer MitraClip@Implantation abzuraten (Neuss et al. 2013).

\subsection{Zielsetzung}

Zahlreiche Studien konnten einen positiven Einfluss einer minimalinvasiven Mitralklappenreparatur mittels MitraClip( auf klinische und linksventrikuläre Parameter im Sinne eines linksventrikulären reversen Remodellings nachweisen (Schillinger et al. 2013; Puls et al. 2014b). Jedoch wurden die Langzeitauswirkungen einer MitraClip@-Implantation auf eine vorbestehende sekundäre pulmonale Hypertonie mit Rechtsherzinsuffizienz bisher nicht in einer größeren Patientenkohorte untersucht. Die Beschreibung eines reversen rechtsventrikulären Remodellings ist bisher ebenfalls nicht erfolgt.

Ziel dieser Arbeit ist die Analyse des Einflusses einer MitraClip@-Implantation auf die Rechtsherzparameter von Patienten mit schwerer Mitralinsuffizienz und begleitender pulmonaler Hypertonie sowie sekundärer Rechtsherzinsuffizienz. Da das Vorhandensein einer pulmonalen Hypertonie seit Langem als Mortalitätsprädiktor bei schwerer Ml sowie auch als relevanter Risikofaktor für die konventionelle Herzchirurgie bekannt ist, soll darüber hinaus untersucht werden, ob sich Veränderungen der Rechtsherzparameter infolge der MitraClip@-Implantation auf das Überleben der Patienten auswirken. 


\section{Material und Methoden}

\subsection{Studiendesign}

Wir führten eine prospektive, multizentrische Verlaufsbeobachtung bei 70 Patienten durch, die in den Herzzentren der Universitätsmedizin Göttingen und des Universitätsklinikums Hamburg-Eppendorf im Zeitraum von 2009 bis 2011 wegen einer schweren Mitralinsuffizienz mittels MitraClip $\odot$ behandelt wurden und begleitend an einer pulmonalen Hypertonie mit klinischen Zeichen einer sekundären Rechtsherzinsuffizienz litten. Ein weiteres Einschlusskriterium war das Vorliegen eines 1-Jahres-Follow-Ups inklusive einer Echokardiographie.

Alle Patienten gaben nach der Aufklärung ihre schriftliche Einwilligung in die Studie, klinische und epidemiologische Daten wurden pseudonymisiert wissenschaftlich ausgewertet. Das positive Votum der Ethikkommission liegt vor. Im Folgenden wird stellvertretend für beide Zentren das Vorgehen im Herzzentrum der Universitätsmedizin Göttingen erläutert.

\subsection{Präinterventionelle Diagnostik und Patientenselektion}

Patienten mit hochgradiger Mitralklappeninsuffizienz, die für eine Behandlung mittels MitraClip@-Verfahren in Frage kamen, wurden nach erfolgter Anamnese vor dem Eingriff mit transthorakaler und transösophagealer Echokardiographie untersucht. Zusätzlich wurde von den Patienten vor dem Eingriff eine spezielle Anamnese erhoben, bei der der Krankheitsverlauf und die kardiologisch bedingten Hospitalisierungen im letzten Jahr im Vordergrund standen. Dabei wurde auch ein standardisierter Sechs-Minuten-Gehtest mit den Patienten durchgeführt, die Vitalparameter wurden dokumentiert und eine Blutentnahme zur Bestimmung von NT-pro BNP durchgeführt.

Außerdem wurden eine Koronarangiographie sowie eine linksventrikuläre Angiographie durchgeführt. Die Befunde dieser Untersuchungen wurden im Anschluss in einem sogenannten Heart Team, bestehend aus einem Herzchirurgen und einem interventionellen Kardiologen, besprochen. Gemäß den gültigen Leitlinien (Vahanian et al. 2012) und unter Zuhilfenahme der bewährten kardiochirurgischen Risikoscores, u.a. EuroSCORE und STSScore, wurde die Indikation überprüft und die geeigneten Behandlungsoptionen abgewogen.

Prozedurassoziierte Daten, wie z.B. die Dauer des Eingriffs und die Durchleuchtungszeit wurden dokumentiert. Vor der Entlassung wurden die Patienten erneut mittels transthorakaler Echokardiographie untersucht. 
In die Studie eingeschlossen wurden volljährige Patienten, bei denen zusätzlich zur hochgradigen Mitralklappeninsuffizienz eine sekundäre pulmonale Hypertonie mit klinischen Zeichen der Rechtsherzinsuffizienz vorlag. Um den pulmonal-arteriellen Druck messen zu können, mussten die Patienten eine in der Echokardiographie dopplerbare Trikuspidalinsuffizienz aufweisen.

Patienten, bei denen andere Ursachen für die pulmonale Hypertonie identifiziert werden konnten, wie z.B. chronische Thrombembolien oder unklare, multifaktorielle Mechanismen, wurden von der Studie ausgeschlossen. Patienten, von denen keine auswertbaren, echokardiographischen Daten vor der MitraClip@-Implantation, vor der Klinikentlassung nach erfolgreicher Implantation und zwölf Monate nach diesem Eingriff vorlagen, wurden ebenfalls von der Studie ausgeschlossen.

\subsection{Follow-Up}

$12 \pm 2$ Monate nach dem Eingriff wurden die Patienten zu einer Nachsorge-Untersuchung einbestellt. In der Regel wurde diese Untersuchung in der Abteilung Kardiologie und Pneumologie der Universitätsmedizin Göttingen (bzw. für die Hamburger Kohorte im Universitätsklinikum Hamburg-Eppendorf) durchgeführt. Eine spezielle Anamnese, eine klinische Untersuchung, eine Feststellung der Vitalparameter, ein standardisierter SechsMinuten-Gehtest, ein EKG, eine Laboruntersuchung des NT-pro BNP im Blut des Patienten sowie eine echokardiographische Untersuchung waren Bestandteile des Follow-Ups. Patienten, die nicht hinreichend mobil waren oder aus anderen Gründen nicht in der Lage waren, nach Göttingen zu kommen, wurden in kardiologischen Praxen vor Ort untersucht.

Bei Patienten, die im Herzzentrum der Universitätsmedizin Göttingen behandelt wurden, wurde eine solche Nachsorgeuntersuchung zusätzlich nach $6 \pm 2$ Monaten durchgeführt. Zusätzlich wurden alle Patienten im September 2013 telefonisch kontaktiert. Dabei wurde der im Anhang angefügte Fragebogen genutzt. 


\subsection{Methoden der klinischen Datenerhebung}

In den folgenden Abschnitten sollen die genauen Methoden zur Erhebung der klinischen Daten aufgelistet und erläutert werden.

\subsubsection{Klinische Untersuchung}

Die klinische Untersuchung der Patienten umfasste vor allem Perkussion und Auskultation des Thorax. Dies diente der Suche nach Zeichen eines Lungenödems als Hinweis auf ein Linksherzversagen. Darüber hinaus wurde dokumentiert, ob bei den Patienten Unterschenkelödeme oder eine Halsveneneinflussstauung als Zeichen eines Rechtsherzversagens vorlagen.

\subsubsection{New York Heart Association (NYHA) Klassifikation}

Die NYHA Klassifikation dient der Einteilung der Schwere einer Herzinsuffizienz gemäß der subjektiven Symptomatik der Patienten. Sie umfasst vier Stadien: Stadium I-Patient mit Beschwerdefreiheit und normaler körperlicher Belastbarkeit, Stadium II-Patient mit Beschwerden bei stärkerer körperlicher Belastung, Stadium III-Patient mit Beschwerden bei leichter körperlicher Belastung, Stadium IV-Patient mit Beschwerden in Ruhe (Herold 2015). Bei den genannten Beschwerden handelte es sich vor allem um Dyspnoe und Schwindel. Der NYHA-Status der Patienten wurde vor Eingriff sowie bei dem Follow-Up $6 \pm 2$ Monate beziehungsweise $12 \pm 2$ Monate nach Eingriff erhoben.

\subsubsection{Sechs-Minuten-Gehtest (6MWT)}

Der Sechs-Minuten-Gehtest dokumentiert die Gehstrecke, die Patienten innerhalb von sechs Minuten in einer Ebene zurücklegen. Dies entspricht einer submaximalen Belastung, da die Patienten ihre Laufgeschwindigkeit selber wählen und z.B. während des Tests pausieren dürfen. Damit ist der Test geeignet, die Leistungsfähigkeit der Patienten besonders im Alltag zu simulieren und zu erfassen (American Thoracic Society 2002). Er wurde sowohl vor der MitraClip@-Implantation als auch $6 \pm 2$ Monate beziehungsweise $12 \pm 2$ Monate danach durchgeführt. 


\subsubsection{Minnesota Living with Heart Failure Questionnaire (MLHFQ)}

Ziel des MLHFQ ist es, die Auswirkungen der Herzerkrankung auf die Lebensqualität der betroffenen Patienten zu quantifizieren. Der Fragebogen besteht aus 21 Fragen, die sich unter anderem auf physische und psychische Belastbarkeit, soziale Interaktion, sexuelle Aktivität sowie finanzielle Belastung durch die Krankheit beziehen. Dabei betrachtet jede Frage ausschließlich den Zeitraum der letzten vier Wochen. Der Antwort des Patienten auf jede Frage kann ein Zahlenwert von Null bis Fünf zugeordnet werden. Die daraus gebildete Summe entspricht einem Parameter für die subjektiv vom Patienten empfundene Einschränkung der Lebensqualität durch seine Herzerkrankung. Für Patienten mit chirurgischer Klappenreparatur konnte gezeigt werden, dass der MLHFQ anderen Fragebögen wie z.B. dem SF-36 überlegen ist (Supino et al. 2009). Die Befragung der Patienten mit Hilfe dieses Fragebogens wurde vor der Intervention sowie bei dem Follow-Up $6 \pm 2$ Monate beziehungsweise $12 \pm 2$ Monate danach erhoben

\subsubsection{Echokardiographische Diagnostik der Rechtsherzinsuffizienz}

Sämtliche Echokardiographien in der Universitätsmedizin Göttingen erfolgten durch nur eine Ärztin auf zwei verschiedenen Geräten, einem Vivid E9 der Firma General Electric (Fairfield, Connecticut) (Screening und Follow-Up-Untersuchung) und einem ie33 der Firma Philips (Amsterdam, Niederlande) (MitraClip@-Prozeduren). Alle Patienten wurden vor der MitraClip@-Implantation mittels transthorakaler (TTE) sowie transösophagealer (TEE) Echokardiographie untersucht. Das Ergebnis der Prozedur wurde vor Entlassung der Patienten aus der Klinik, mindestens aber 24 Stunden nach der MitraClip@-Implantation mittels TTE kontrolliert. Im Rahmen der Follow-Up-Untersuchung wurde ausschließlich eine transthorakale Echokardiographie durchgeführt. Alle transthorakalen Echokardiographieuntersuchungen wurden zudem digital gespeichert, sodass nachträgliche Auswertungen möglich waren. Anhand dieser Befunde wurde von zwei unabhängigen Untersuchern eine retrospektive echokardiographische Analyse der Rechtsherzparameter durchgeführt. Beide Untersucher waren sowohl zur Person der Patienten als auch zum Zeitpunkt der Untersuchung (vor MitraClip@-Implantation, vor Klinikentlassung nach MitraClip@Implantation und im Rahmen der Follow-Up-Untersuchung $12 \pm 2$ Monate später) verblindet. Die Analyse erfolgte gemäß den Empfehlungen der amerikanischen (American College of Cardiology/ American Heart Association) und europäischen (European Society of Cardiology) Fachgesellschaften mit besonderem Augenmerk auf Rechtsherz-Parameter statt (Bonow et al. 2006; Galie et al. 2009; Vahanian et al. 2012; Nishimura et al. 2014). 
Zur Diagnostik einer pulmonal-arteriellen Hypertonie sowie Bestimmung rechtsventrikulärer Parameter bietet sich die Echokardiographie als nicht-invasive Standardmethode an. Sie ermöglicht die nicht-invasive Bestimmung des systolischen pulmonal-arteriellen Druckes, der sehr gut mit invasiv bestimmten Parametern korreliert (Ghio et al. 2013). Zur Abschätzung wird die Bestimmung der maximalen Geschwindigkeit des Trikuspidalinsuffizienz-Jets (Vmax) mittels continuous-wave Doppler genutzt. Das Einsetzen des gemessenen Wertes in die modifizierte Bernoulli-Gleichung (PAPsyst $=4^{*}\left(V_{\max }\right)^{2}+$ mittlerer rechtsatrialer Druck) ergibt dann einen Schätzwert für den systolischen pulmonal-arteriellen Druck (Huber und Glaser 2013). Für den rechtsatrialen Druck wird ein Wert von $5 \mathrm{mmHg}$ bei vollständigem Kollaps der Vena cava inferior, ein Wert von $10 \mathrm{mmHg}$ bei einem partiellen und ein Wert von $15 \mathrm{mmHg}$ bei fehlendem Kollaps verwendet (Kircher et al. 1990). Gemäß den aktuellen Leitlinien der ESC und AHA/ACC entspricht eine pulmonale Hypertonie einem pulmonalarteriellem Druck größer 50 mmHg in Ruhe (Galie et al. 2009; Nishimura et al. 2014).

Bei der Bestimmung rechtsventrikulärer Parameter stellt die im Vergleich zum linken Ventrikel sehr komplexe Morphologie des rechten Ventrikels ein Problem dar. Eine auf zweidimensionalen Flächenmessungen beruhende Einschätzung der rechtsventrikulären Ejektionsfraktion wird gemäß aktueller Leitlinien (Rudski et al. 2010) nicht empfohlen, da die vorhandenen mathematischen Rechenmodelle der komplexen Geometrie des rechten Ventrikels nicht gerecht werden, sodass es leicht zu Fehleinschätzungen kommt (Nagel et al. 1996; Zakeri et al. 2014). Da der rechte Ventrikel überwiegend über longitudinale Muskelfasern verfügt, die zu einer deutlichen baso-apikalen Bewegung des Trikuspidalklappenringes während der Systole führen, korreliert der im M-Mode erhobene TAPSE-Wert (Tricuspid Annular Plane Systolic Excursion) sehr gut mit der rechtsventrikulären Ejektionsfraktion und wird daher gemäß aktueller Leitlinien zur routinemäßigen Einschätzung der rechtsventrikulären Funktion empfohlen (Rudski et al. 2010) (vgl. auch untenstehende Abb. 6). Baso-apikale Bewegungen des Trikuspidalklappenringes unter $16 \mathrm{~mm}$ sind dabei als pathologisch zu werten (Miller et al. 2004). 

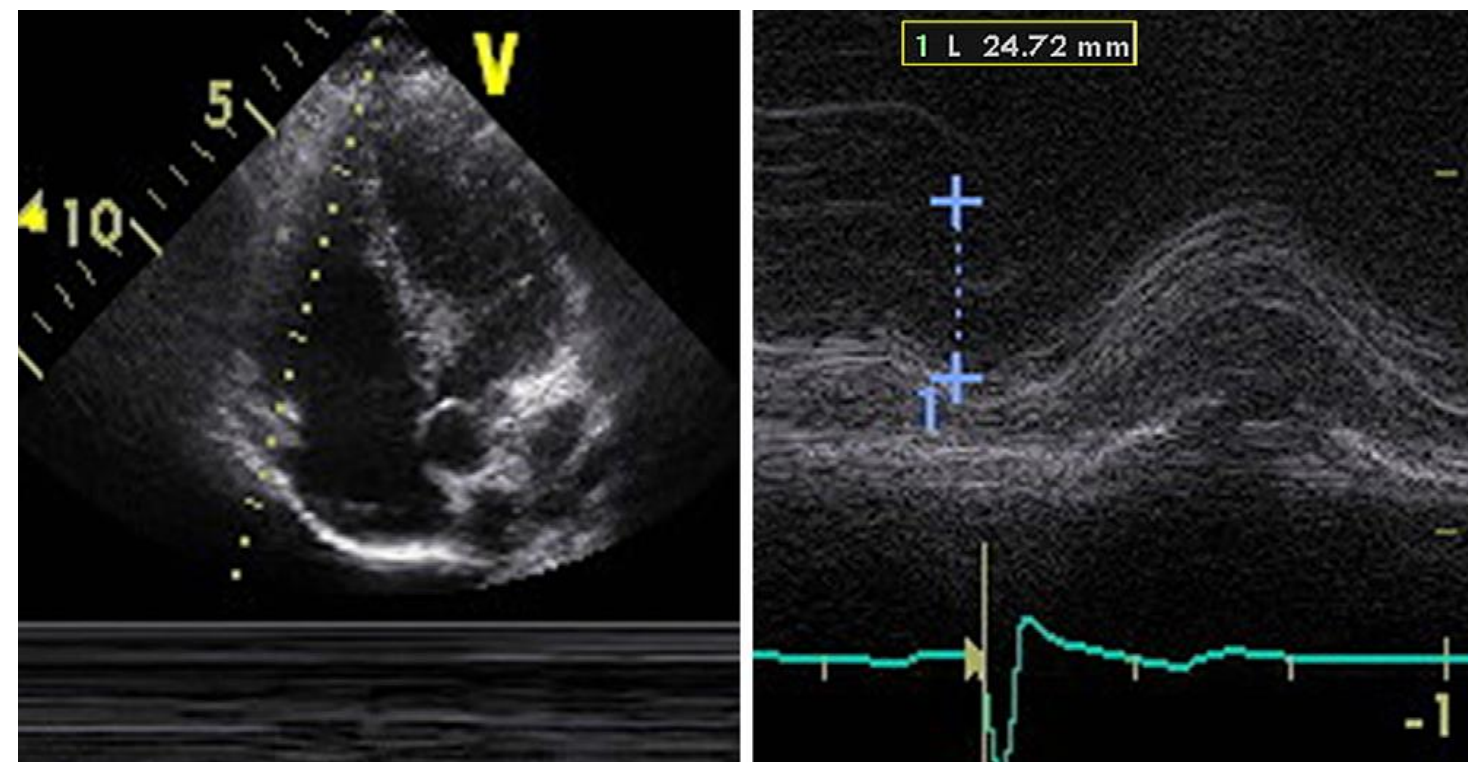

Abbildung 6: Platzierung des M-Mode Cursors am lateralen Rand des Trikuspidalklappenringes im apikalen 4-Kammerblick (links) und Bestimmung der systolischen, baso-apikalen Bewegung des Trikuspidalklappenringes im M-Mode (rechts) (aus Rudski et al. 2010)

Weiterhin wird bei Patienten mit Verdacht auf Rechtsherzinsuffizienz die Bestimmung der rechtsventrikulären Dimensionen auf Höhe der RV-Basis (RVD1), des mittleren Kavums (RVD2), sowie der longitudinalen Ausdehnung (RVD3) (im 4-Kammerblick) empfohlen (Rudski et al. 2010) (vgl. auch Abb. 7).

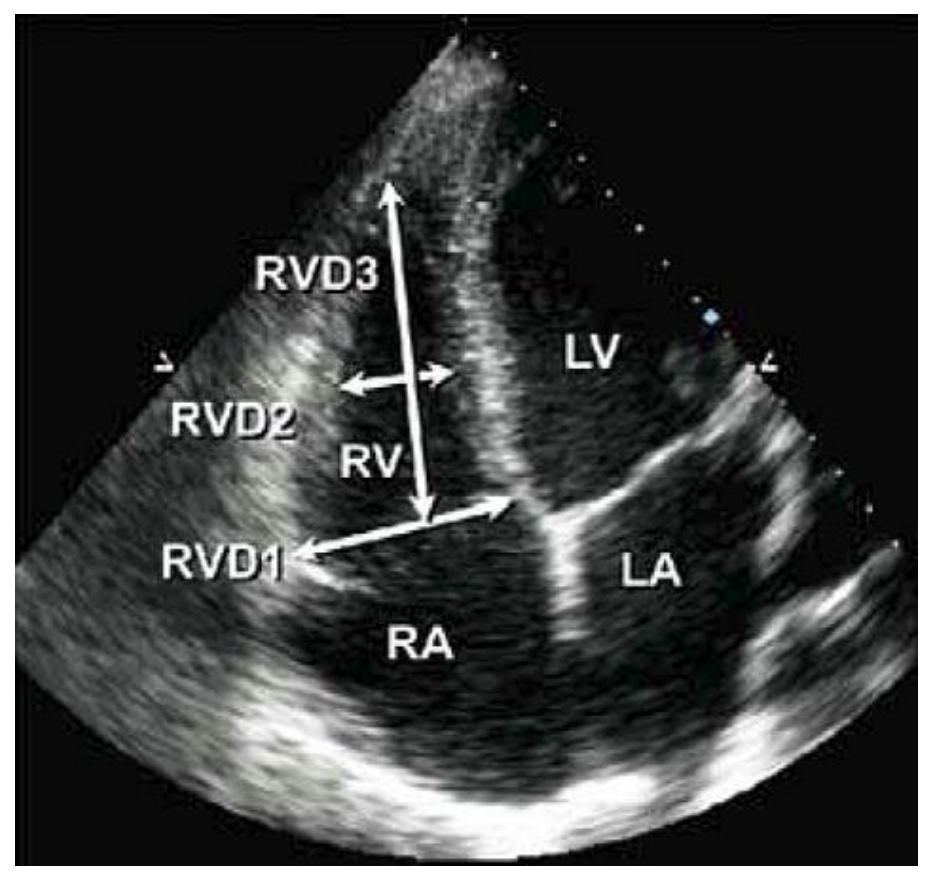

Abbildung 7: Ausmessung der rechtsventrikulären Dimensionen im 4-Kammerblick (aus Rudski et al. 2010) 


\subsubsection{Herzkatheter}

Weiterer Bestandteil der Baseline-Diagnostik vor MitraClip@-Implantation ist die Herzkatheter-Untersuchung. Sie dient der Feststellung des Status der Koronararterien und kann so weitere Hinweise auf die Ätiologie der Mitralinsuffizienz sowie deren Therapieoptionen geben (Vahanian et al. 2012).

\subsubsection{European System for Cardiac Operative Risk Evaluation (EuroSCORE)}

Der EuroSCORE ermöglicht eine Risikoabschätzung der 30-Tages-Mortalität von Patienten, die sich einer herzchirurgischen Operation unterziehen. Die Berechnung des EuroSCOREs erfolgte jeweils vor der Besprechung im Heart Team beziehungsweise vor der Intervention über eine im Internet verfügbare Maske (http://euroscore.org/calc.html). Dort müssen verschiedene operative und kardiovaskuläre Risikofaktoren (z.B. Alter, Geschlecht, Diabetes, pulmonale Hypertonie sowie vorherige Operationen an der thorakalen Aorta) eingegeben werden, denen statistisch ermittelte Zahlenwerte zugeordnet werden. Mithilfe mathematischer Algorithmen erfolgt im Anschluss eine Errechnung der genannten 30-TagesMortalität. Seit 2011 ist eine überarbeitete Version des EuroSCOREs, der EuroSCORE II, verfügbar. Unsere Werte wurden jedoch noch gemäß des EuroSCOREs I ermittelt, der als zuverlässiges Instrument angesehen wird, um die 30-Tages-Mortalität von herzchirurgischen Patienten abzuschätzen (Geissler et al. 2000). Darüber hinaus ist ein Vorteil des EuroSCOREs II für Hochrisiko- Patienten umstritten (Di Dedda et al. 2013; Howell et al. 2013).

\subsubsection{Society of Thoracic Surgeons (STS) Score}

Der STS-Score stellt ein weiteres Instrument zur Abschätzung der perioperativen Mortalität bei herzchirurgischen Patienten dar. Dieser erfordert ebenfalls die Eingabe der patientenbezogenen Risikofaktoren. Wie auch der EuroSCORE wurde der STS-Score in unserer Verlaufsbeobachtung vor der Mitraclip@-Implantation berechnet. In Hinblick auf eine isolierte Herzklappenchirurgie besitzt der STS-Score eine höhere Genauigkeit (Wendt et al. 2009). 


\subsection{Das MitraClip@-System}

Das MitraClip@-System setzt sich maßgeblich aus zwei Bestandteilen zusammen, dem steuerbaren Führungskatheter mit Dilatator und dem Clip-Einführsystem, an dessen Ende sich der MitraClip@ selbst befindet. Der Führungskatheter hat eine Weite von 24 French (ca. $8 \mathrm{~mm}$ ) und verjüngt sich auf 22 French (ca. 7,33 mm) am Punkt des transseptalen Überganges (Feldman et al. 2005). Führungskatheter und Dilatator stellen eine adäquate transseptale Verbindung zwischen peripherer Punktionsstelle und dem linken Atrium sicher. Des Weiteren dient der Führungskatheter auch der Steuerung des MitraClips@. Dieser wird an der Spitze des Clip-Einführsystems im linken Atrium über der Mitralklappe ausgerichtet und anschließend über den linken Ventrikel in Höhe der Mitralklappensegel zur Verringerung der Mitralinsuffizienz vorgebracht. Der MitraClip@ an sich besteht aus einer Cobalt-ChromLegierung, die mit einem Polyestergewebe überzogen ist. Der Clip hat zwei Arme, die jeder ungefähr $8 \mathrm{~mm}$ lang und $4 \mathrm{~mm}$ breit sind (Jilaihawi et al. 2011) (siehe Abbildung 8).
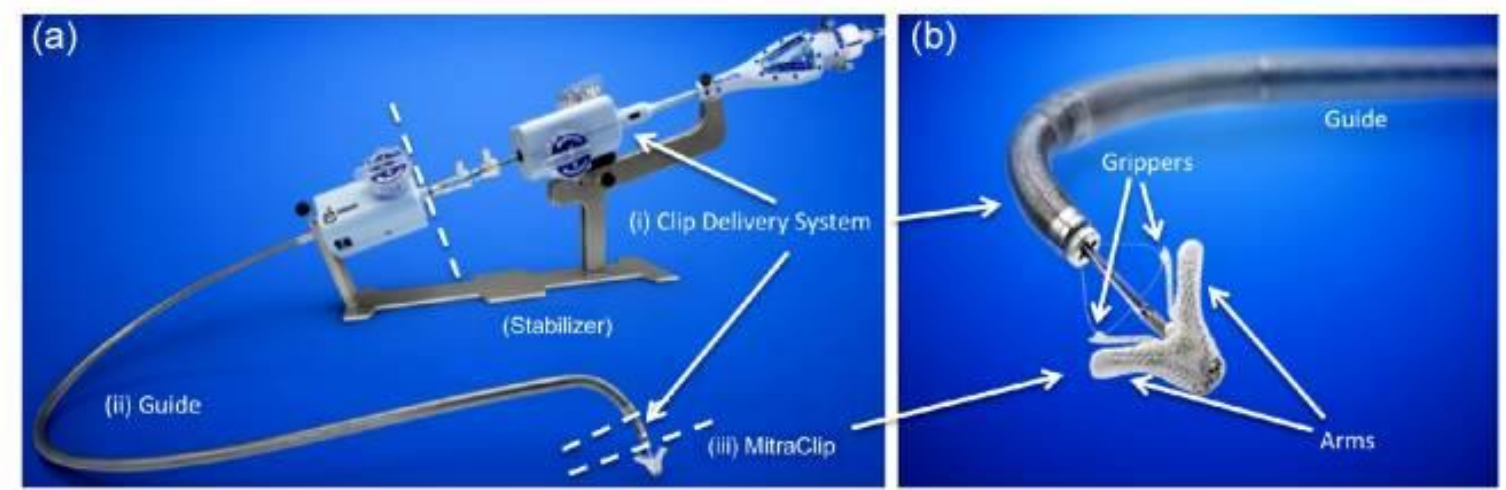

Abbildung 8: Das MitraClip@-System (aus Jilaihawi et al. 2011)

\subsection{Das MitraClip@-Verfahren}

Wie bei jedem interventionellen Eingriff ist vor der MitraClip@-Implantation das Gerinnungsmanagement von Bedeutung. Standard ist die einmalige Gabe von $100 \mathrm{mg}$ ASS ein Tag vor dem Eingriff. Generell sollte für den Eingriff eine International Normalized Ratio (INR) von unter 1,7 bzw. ein Quick-Wert von 50\% angestrebt werden. Patienten, die vor dem Eingriff mit einem Vitamin-K-Antagonisten antikoaguliert wurden, erhalten vor dem Eingriff eine Umstellung ihrer Antikoagulation auf Heparin, welche am Tag der MitraClip@Implantation pausiert wird. Abgesehen davon wird der Patient vor dem Eingriff mit einem Harnblasenkatheter versorgt. 
Die mehrfach vorbeschriebene (Feldman et al. 2005; Feldman et al. 2009; Jilaihawi et al. 2011) MitraClip@-Implantation erfolgt in der Universitätsmedizin Göttingen im Herzkatheterlabor bzw. dem Hybrid-OP. Das behandelnde Team besteht aus einem kardiologischen Anästhesisten, einem Echokardiographeur (der auch die Echokardiographien im Rahmen des Baseline-Screenings durchgeführt hat), einem Mitarbeiter von Abbott Vascular, zwei erfahrenen interventionellen Kardiologen und spezialisiertem Pflegepersonal. Die Anordnung/ der Aufbau des Hybrid-OPs entspricht dabei der Abbildung 9.

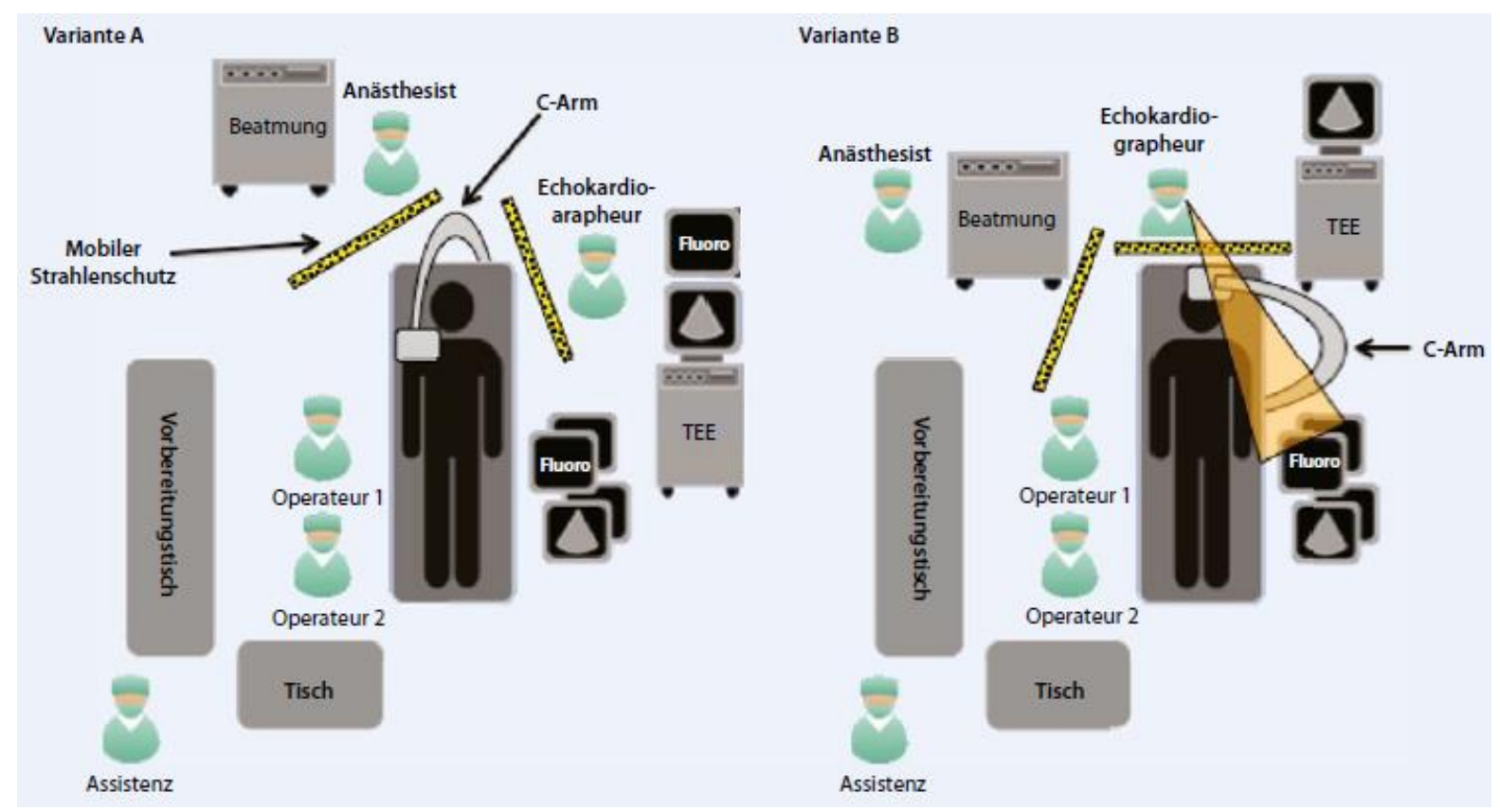

Abbildung 9: Zwei mögliche Anordnungen im Herzkatheterlabor (aus Boekstegers et al. 2013)

Sobald der Patient auf dem Herzkathetertisch liegt, wird er von dem anwesenden Facharzt für Anästhesie mit einem zentralen Venenkatheter, einem peripheren Venenzugang sowie einer arteriellen Blutdruckmessung versorgt. Darauf erfolgt die endotracheale Intubation für die während der Intervention erforderliche Vollnarkose. Darüber hinaus wird nun, vor dem eigentlichen Eingriff, eine erneute transösophageale Echokardiographie durchgeführt, um die Befunde des vorangegangenen Baseline-Screenings zu kontrollieren und zu bestätigen. Mittels 3D-TEE kann auch die genaue Anatomie der Mitralklappe erneut evaluiert werden. Mit Hilfe eines Farbdopplers werden Ursprung und Schwere der Mitralinsuffizienz kontrolliert (vgl. Abb. 10). 


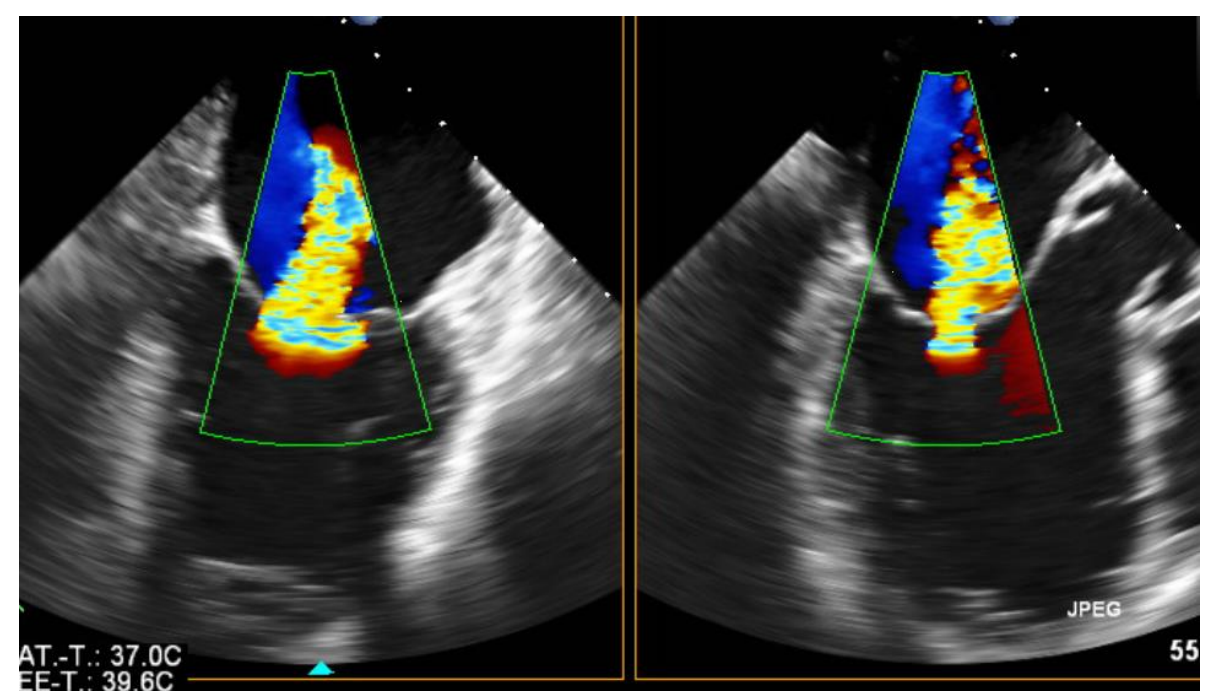

Abbildung 10: Darstellung und Evaluation der hochgradigen sekundären $\mathrm{Ml}$ in der zweidimensionalen Echokardiographie zu Prozedurbeginn (aus Puls und Schillinger 2016)

Um einen Zugangsweg zum Herzen zu schaffen, erfolgt zu Beginn der MitraClip@Implantation die Punktion der rechten Vena femoralis gemäß Seldinger-Technik. Nach Vorschub eines Führungsdrahes in das rechte Atrium wird mit Hilfe einer Transseptalnadel das Vorhofseptum im Bereich der Pars membranacea punktiert (vgl. Abb. 11a). Nun wird der Patient heparinisiert, um Thrombenbildung zu vermeiden. Es folgt die Dehnung der septalen Punktionsstelle mittels eines Dilatators, der zusammen mit dem Führungskatheter über einen besonders steifen Führungsdraht unter echokardiographischer und röntgenologischer Kontrolle vorsichtig in das linke Atrium vorgeschoben wird. Bei zu schnellem Vorschieben besteht die Gefahr, umliegendes Gewebe zu verletzen oder Gefäße zu perforieren. Im Anschluss werden Führungsdraht und Dilatator zurückgezogen. Hierbei muss darauf geachtet werden, dass der Dilatator nicht die Wand des Führungskatheters berührt, was zur Bildung eines Vakuums und somit zu einer Luftembolie führen könnte. Nun wird das ClipEinführsystem mit dem MitraClip@ an seiner Spitze unter röntgenologischer Kontrolle durch den Führungskatheter bis in den linken Vorhof vorgeschoben (vgl. Abb. 11b). 

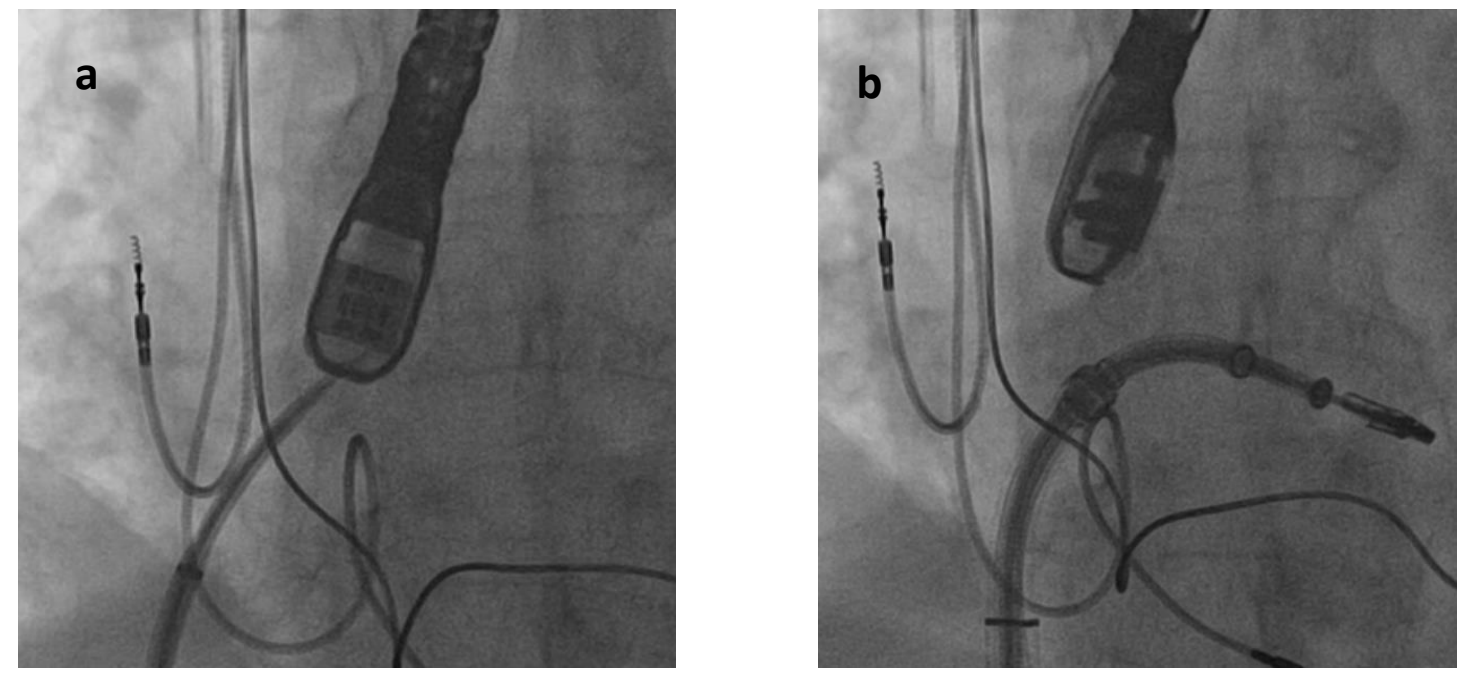

Abbildung 11: Fluoroskopische Darstellung der Punktion des interatrialen Septums (a) sowie des Vorbringens des auf den steuerbaren Führungskatheter montierten Clips in das linke Atrium (b) (aus Puls und Schillinger 2016)

Wenn sich der MitraClip@ im TEE innerhalb des linken Atriums darstellt und sich dabei in ausreichendem Abstand zu Vorhofwand und Klappenebene befindet, erfolgt die Ausrichtung des MitraClips@ $\odot$. Er sollte zentral im Insuffizienzjet, axial und senkrecht zur Mitralklappe ausgerichtet werden (siehe Abbildung 12).

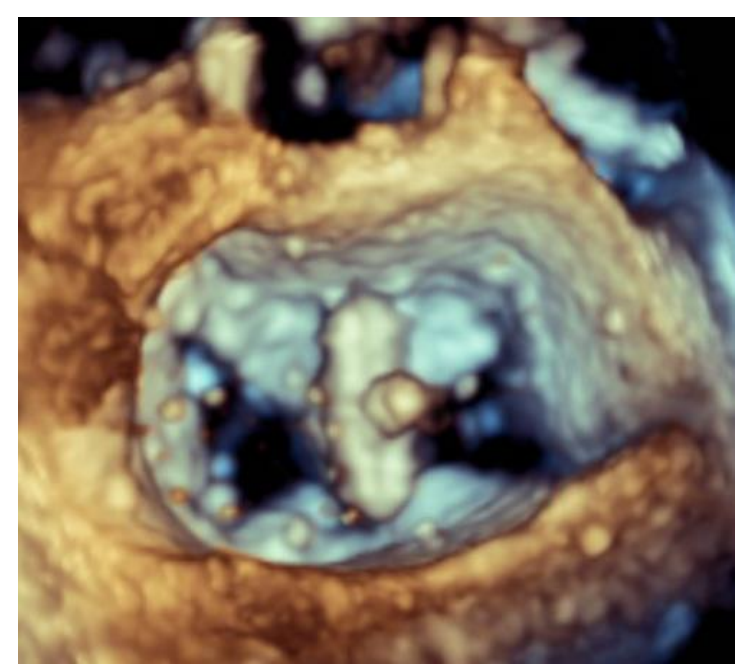

Abbildung 12: Linksatriale echokardiographische Ausrichtung des MitraClips@ unter TEEKontrolle (aus Puls und Schillinger 2016)

Sobald dies gelungen ist, werden nun die Arme des MitraClips $\left(\subset\right.$ auf bis zu $180^{\circ}$ geöffnet und dieser wird in den linken Ventrikel bis $2 \mathrm{~cm}$ unterhalb der Klappenebene vorgeschoben. Jetzt können die sogenannten Greifer auf ungefähr $120^{\circ}$ geöffnet werden und der MitraClip@ wird sehr vorsichtig zurückgezogen, wobei anteriores und posteriores Segel der Mitralklappe zwischen Arm und Greifer des MitraClips@ gefangen werden sollen (vgl. Abb. 13). 


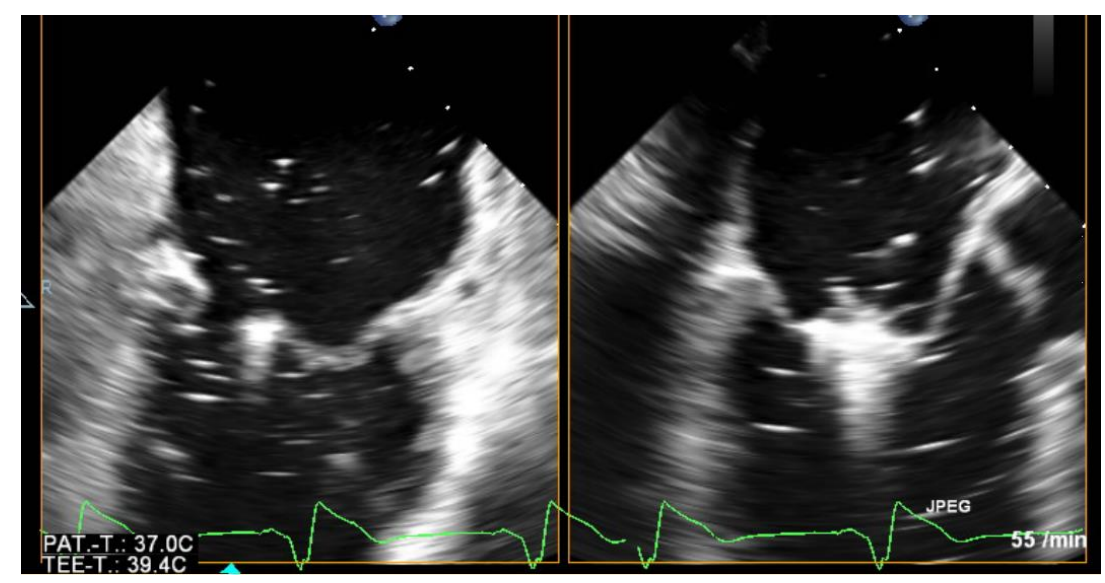

Abbildung 13: Schließen des Clips unter echokardiographischer Sicht, während beide Segel auf den Clip-Armen aufliegen (aus Puls und Schillinger 2016)

Der MitraClip(C) wird geschlossen, Lage sowie Reduktion des Insuffizienzjets mittels Farbdoppler kontrolliert, eine Stenosierung der Mitralklappe mittels Bestimmung des Gradienten über der Klappe ausgeschlossen. Wenn eine zufriedenstellende Reduktion der Mitralinsuffizienz gelungen ist, kann der Clip freigesetzt werden (Feldman et al. 2005). Das Ergebnis sollte erneut echokardiographisch kontrolliert werden (siehe Abbildung 14).

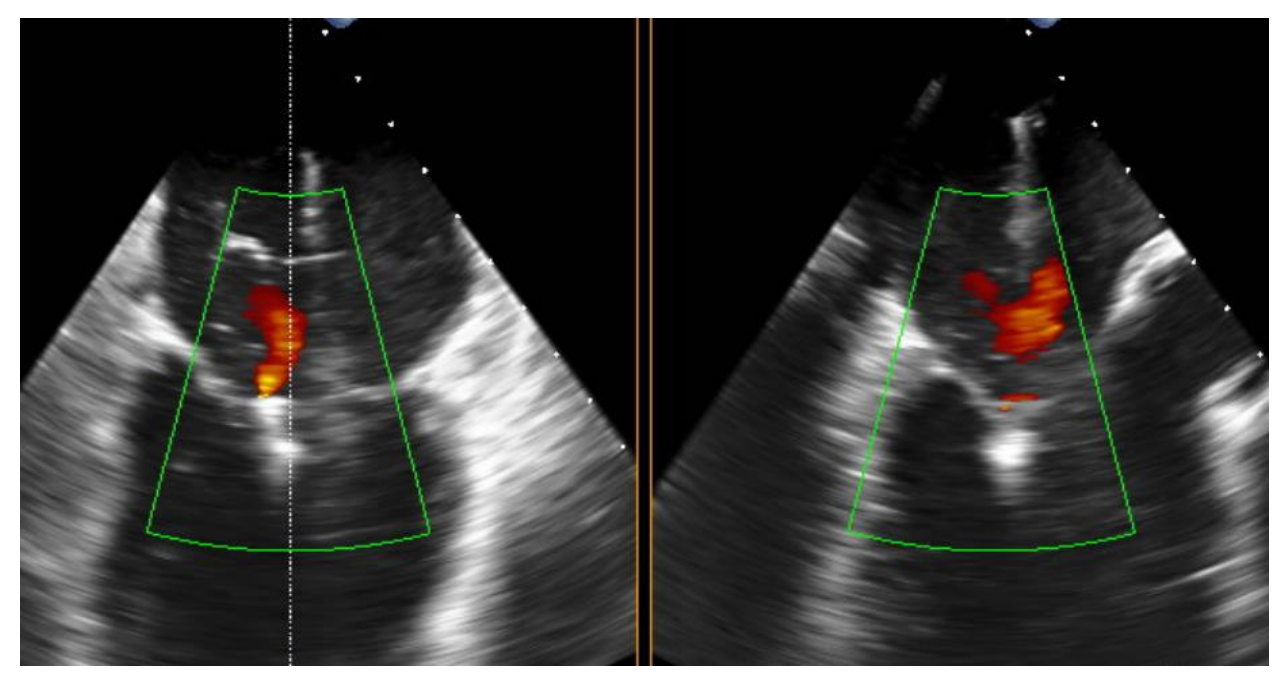

Abbildung 14: Minimale residuale MI in der biplanen TEE-Darstellung nach Clip-Platzierung (aus Puls und Schillinger 2016) 
Infolge der Clip-Implantation entsteht eine Mitralklappe mit zwei Öffnungen (vgl. Abb. 15).

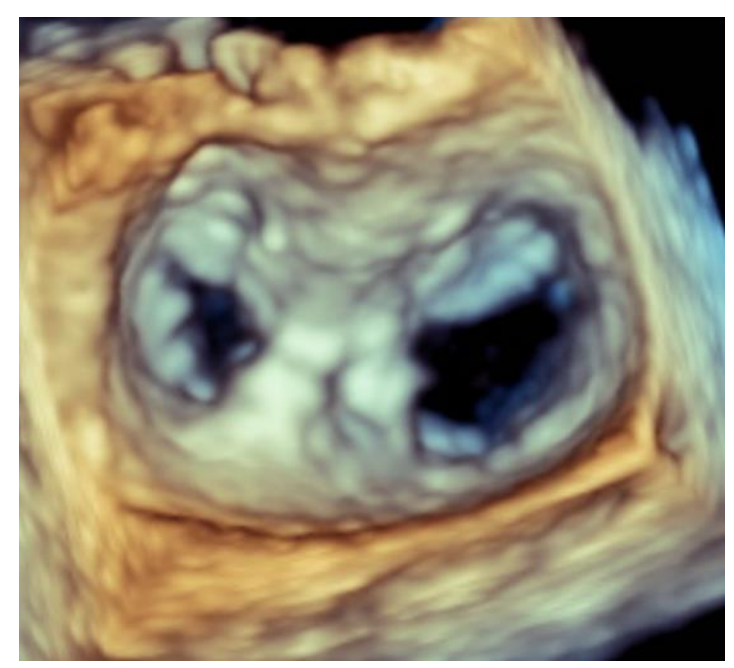

Abbildung 15: Mitralklappe mit 2 Öffnungen (double-orifice) in der 3D-TEE nach ClipImplantation (aus Puls und Schillinger 2016)

Sofern Ätiologie und Anatomie der Mitralinsuffizienz genau evaluiert worden sind, können sogar mehr als zwei MitraClips $\subseteq$ sicher und mit minimaler Gefahr einer Mitralstenose implantiert werden (Paranskaya et al. 2013). Im Falle eines hämodynamisch relevanten Vorhofseptumdefekts besteht die Möglichkeit des Verschlusses mit einem VorhofseptumOkkluder. Nun können Clip-Einführsystem sowie Führungskatheter zurückgezogen und die Punktionsstelle mittels eines vorgelegten ProGlide Verschlusssystems der Firma Abbott verschlossen werden. Eine Alternative ist der Verschluss der Punktionsstelle mittels einer ZNaht, die jedoch für die Patienten schmerzhafter und kosmetisch weniger schön ist (Rüter et al. 2013).

In aller Regel erfolgt noch im Herzkatheterlabor die Extubation. Alle weiteren Zugänge werden erst nach 24 Stunden gezogen. Die Fäden der Hautnaht können ggf. nach 10 Tagen gezogen werden.

Zur notwendigen Thrombozytenaggregationshemmung nach dem Eingriff werden dem Patienten einmalig $300 \mathrm{mg}$ Clopidogrel verabreicht. Für die nächsten vier Wochen erhält der Patient eine tägliche Dosis von $75 \mathrm{mg}$ Clopidogrel. Standardmäßig werden Patienten für mindestens sechs Monate nach der MitraClip@-Implantation zusätzlich mit einer täglichen ASS-Dosis von $100 \mathrm{mg}$ behandelt (Feldman et al. 2011b). Patienten, die bereits vor dem Eingriff einen Vitamin-K-Antagonisten eingenommen haben, bekommen lediglich in den ersten vier Wochen eine zusätzliche Gabe von 100 mg ASS täglich. 


\subsection{Statistik}

Die statistische Auswertung erfolgte mit Unterstützung durch die Abteilung für Medizinische Statistik der Universitätsmedizin Göttingen.

Alle Ergebnisse dieser Arbeit sind (je nach Vorhandensein einer Normalverteilung) entweder als Median \pm Interquartilabstand oder als Mittelwert \pm der Standardabweichung (SD) angegeben.

Kontinuierliche Variablen wurden (in Abwesenheit einer Normalverteilung) mittels Wilcoxon matched pairs test für gepaarte Stichproben verglichen. Weiterhin wurden Überlebenszeitanalysen durchgeführt, mittels Kaplan-Meier-Kurven visualisiert und mittels eines logrank Tests verglichen. Dabei wurde jeweils ein $p$-Wert $<0,05$ als statistisch signifikant gewertet.

Zur statistischen Auswertung wurden die Statistikprogramme SigmaStat 2.0 (Jandel Scientific) sowie graph pad prism Version 4.0 verwendet. 


\section{Ergebnisse}

Zwischen 2009 und 2011 wurden insgesamt 321 Patienten in Göttingen ( $\mathrm{n}=84)$ und Hamburg $(n=237)$ gescreent. Die definierten Einschlusskriterien für die vorliegende Studie erfüllten dabei 31 Patienten im Zentrum Göttingen und 39 Patienten im Zentrum Hamburg, sodass insgesamt 70 Patienten in die Studie eingeschlossen werden konnten.

\subsection{Demographische und klinische Baseline Charakteristika}

Das durchschnittliche Alter der 70 in die Studie eingeschlossenen Patienten betrug zum Zeitpunkt des Einschlusses 72,5 \pm 9 Jahre. 66\% $(n=46)$ dieser Patienten waren männlich. Mit $71 \%(n=50)$ überwog in dieser Patientenkohorte die sekundäre Ätiologie der Mitralinsuffizienz. Alle Patienten litten an einer symptomatischen Herzinsuffizienz und befanden sich entsprechend zu 94\% $(n=66)$ in den NYHA-Stadien 3 und 4. Darüber hinaus wiesen die Patienten zum Zeitpunkt der präinterventionellen Untersuchung zahlreiche Komorbiditäten auf. Besonders häufig lagen eine eingeschränkte Nierenfunktion (GFR<60 $\left.\mathrm{ml} / \mathrm{min} / 1,73 \mathrm{~m}^{2} ; 66 \%, \mathrm{n}=46\right)$ und Vorhofflimmern $(64 \%, \mathrm{n}=45)$ vor. Jedoch bestand auch häufig eine koronare Herzerkrankung $(36 \%, n=25)$ oder Diabetes mellitus $(27 \%, n=19)$. Entsprechend den erhobenen hohen NYHA-Stadien und der zahlreichen Begleiterkrankungen betrug der mittlere logistische EuroSCORE $30 \pm 12 \%$ beziehungsweise der mittlere STS-Score $10 \pm 4 \%$. Somit handelte es sich bei der Studienkohorte um ein chirurgisches Hochrisikokollektiv. Im Sechs-Minuten-Gehtest wurde eine mittlere Gehstrecke von 213 m ermittelt (vgl. Tabelle 1). 
Tabelle 1: Basisdemographische Charakteristika des Gesamtkollektivs $(n=70)$

\section{Mittelwert \pm Standardabweichung}

Alter (Jahre)

$72,5 \pm 9$

Errechnetes peri-operatives Risiko

- Logistischer EuroSCORE I (\%)

$30 \pm 12$

- STS Score (\%)

$10 \pm 4$

Sechs-Minuten-Gehtest (m)

$213 \pm 54$

$n(\%)$

Männliches Geschlecht

$46 / 70(66 \%)$

NYHA-Stadium II

$4 / 70(6 \%)$

NYHA-Stadium III

$43 / 70(61 \%)$

NYHA-Stadium IV

$23 / 70(33 \%)$

Klinische Zeichen der

$70 / 70(100 \%)$

Rechtsherzinsuffizienz

Ätiologie der Mitralinsuffizienz:

- Primär (degenerativ)

20/70 (29\%)

- Sekundär (funktionell)

$50 / 70(71 \%)$

$E F \leq 30 \%$

$42 / 70(60 \%)$

Koronare Herzerkrankung (KHK)

$25 / 70(36 \%)$

Z.n. ACVB-Operation

$25 / 70(36 \%)$

Z.n. Thorakotomie

$30 / 70(43 \%)$

Vorhofflimmern

$45 / 70(64 \%)$

Chron. Niereninsuffizienz

$46 / 70(66 \%)$

- GFR $<30$

$23 / 70$ (33\%)

Diabetes mellitus

$19 / 70(27 \%)$

Z.n. Schlaganfall

$6 / 70(9 \%)$

COPD

19/70(27\%) 


\subsection{Klinischer Erfolg der MitraClip@-Implantation}

Der klinische Erfolg der MitraClip@-Implantation wurde $6 \pm 2$ und $12 \pm 2$ Monate nach der Intervention anhand der Göttinger Patientenkohorte untersucht.

Die mediane subjektive Belastbarkeit der in Göttingen behandelten Patienten vor der Prozedur entsprach der NYHA-Klasse III [III; IV]. Diese verbeserte sich nach 6 Monaten statistisch signifikant auf NYHA-Klasse II [II; III] $(p<0,0001 ; n=24)$ und blieb auch nach 12 Monaten stabil $(p<0,0001 ; n=20)$, ohne dabei erneute Signifikanz im Vergleich zu den Werten 6 Monate nach der Prozedur zu erlangen $(p=0,5 ; n=15)$.

Ebenso stieg die im Sechs-Minuten-Gehtest zurückgelegte Strecke von 280 [100; 350] m vor der MitraClip@-Implantation signifikant auf 335 [200; 400] m nach 6 Monaten ( $p=0,003$; $\mathrm{n}=24)$. Diese Signifikanz konnte mit 320 [200; 430] m nach 12 Monaten im Vergleich zu den Werten vor der Prozdeur gehalten werden $(p=0,04 ; n=23)$, ohne jedoch im Vergleich zu den Werten nach 6 Monaten erneut signifikant zu werden $(p=0,4 ; n=22)$.

Außerdem verringerte sich die Punktezahl im Minnesota Living with Heart Failure Questionnaire signifikant von 48,5 [29;62,5] Punkten auf 35 [16; 50] Punkte ( $p=0,003 ; n=26)$. Auch dieses Ergebnis blieb nach weiteren 6 Monaten stabil bei 29 [13; 42] Punkten $(p=0,002 ; n=25)$. Eine erneute Signifikanz gegenüber den Werten von einem halben Jahr nach der Prozedur konnte ebenfalls nicht erlangt werden $(p=0,2 ; n=26)$.

\subsection{Veränderungen der Rechtsherzparameter vor Klinikentlassung}

Die unmittelbaren Auswirkungen der MitraClip@-Implantation auf das rechte Herz wurden mittels einer transthorakalen Echokardiographie ermittelt. Um einen möglichen Einfluss der Vollnarkose auszuschließen, wurde diese TTE nicht noch im Herzkatheterlabor durchgeführt, sondern mindestens 24 Stunden nach der Prozedur, definitiv aber vor Entlassung des Patienten aus der Klinik.

Im Vergleich zu den präinterventionell erhobenen Daten veränderten sich sowohl basaler als auch mittlerer und longitudinaler Diameter des rechten Ventrikels statistisch nicht signifikant. Ebenso wie die Weite der Vena cava inferior blieben auch die Flächenmessungen von rechtem Vorhof und Ventrikel sowie der RVOT-Diameter unverändert.

Die systolischen und diastolischen Weitenmessungen des Trikuspidalklappenanulus' sowie die Bestimmung der trikuspidalen Regurgitationsfläche zeigten ebenfalls keine signifikanten Veränderungen.

Allerdings lag bereits zum Entlassungszeitpunkt tendenziell eine Verkleinerung der Vena contracta der Trikuspidalinsuffizienz $(\mathrm{TI})$ von $0,77[0,65 ; 0,88] \mathrm{cm}$ auf $0,74[0,59 ; 0,82] \mathrm{cm}$ vor 
$(p=0,06)$. Darüber hinaus zeigte sich eine statistisch signifikante Reduktion der maximalen Geschwindigkeit der Trikuspidalregurgitation von 3,51 [3,21;3,73] m/s auf 3,16 [2,78; 3,42] $\mathrm{m} / \mathrm{s}(\mathrm{p}=0,001)$, des maximalen Gradienten der Trikuspidalregurgitation von 49,28 [41,22; $55,65] \mathrm{mmHg}$ auf $39,94[30,91 ; 46,79] \mathrm{mmHg}(p=0,001)$ und eine Abnahme des systolischen pulmonal-arteriellen Druckes von $60,85[52,15 ; 66,29] \mathrm{mmHg}$ auf $54,00[39,45 ; 57,55] \mathrm{mmHg}$ $(p=0,04)$.

Außerdem konnte eine statistisch signifikante Zunahme der TAPSE von 16,0 [13,0; 20,0] mm auf $20,0[15,0 ; 21,0] \mathrm{mm}(p=0,002)$ direkt nach der Intervention gezeigt werden.

Die statistisch signifikanten Veränderungen sind in Abbildung 16 veranschaulicht.

\subsection{Veränderungen der Rechtsherzparameter nach 12 Monaten}

Alle TTEs zum Nachweis langfristiger Auswirkungen der MitraClip@-Implantation wurden 12 Monate \pm 2 Monate nach der Intervention durchgeführt.

Unmittelbar nach dem Eingriff aufgetretene Veränderungen blieben auch ein Jahr später stabil. So setzten sich die bereits bei Klinikentlassung statistisch signifikanten Abnahmen von maximaler Geschwindigkeit der Trikuspidalregurgitation, maximalem Gradienten der Trikuspidalregurgitation (TR) und systolischem pulmonal- arteriellem Druck wie in Abbildung 10 dargestellt fort. Die maximale Geschwindigkeit der TR sank auf 3,15 [2,77; 3,27] m/s, der maximale Gradient über der TR auf 39,69 [30,69; 42,77] $\mathrm{mmHg}$ und der systolische pulmonal-arterielle Druck sank auf 46,0 [39,5; 54,3] $\mathrm{mmHg}$. Damit blieben alle Veränderungen gegenüber den Baseline Werten signifikant $(p(V \max T R)=0,001 ; p($ Peak $T R)=0,001 ; p(P A P s y s t)=0,03)$, konnten allerdings im Vergleich zu den Messungen $z u$ Klinikentlassung keine erneute Signifikanz gewinnen.

Gleiches gilt für die TAPSE, die sich auch innerhalb der ersten 12 Monate nach Intervention weiter auf im Median 18,88 [17,0; 21,0] mm erhöhte (im Vergleich zum Ausgangswert $p=0,001)$.

Die langsame Reduktion des RVOT Diameters und die Weite der Vena contracta der Trikuspidalinsuffizienz setzten sich fort, um nach 12 Monaten eine statistische Signifikanz im Vergleich mit den präinterventionellen Werten zu erreichen (RVOT Diameter von 3,51 [3,09; $3,90] \mathrm{cm}$ auf $3,30[3,14 ; 3,90] \mathrm{cm}, p=0,01$; Vena contracta von $0,77[0,64 ; 1,02] \mathrm{cm}$ auf 0,74 $[0,61 ; 0,93] \mathrm{cm}, p=0,01)$. Diese Veränderungen sind in Abbildung 16 genau dargestellt. 
A

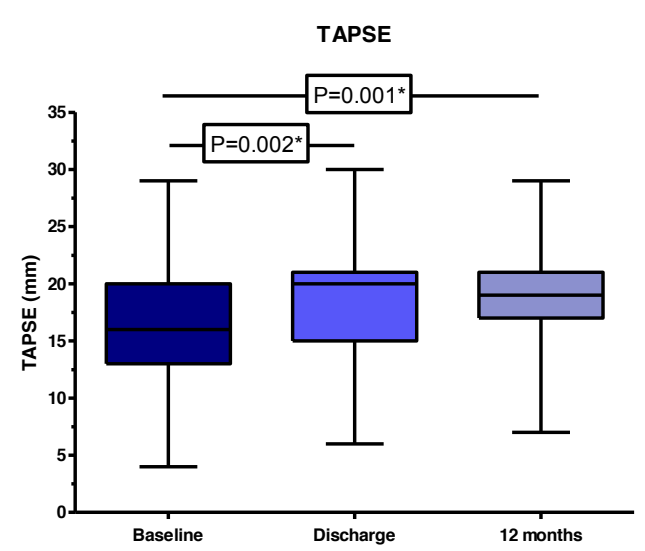

C

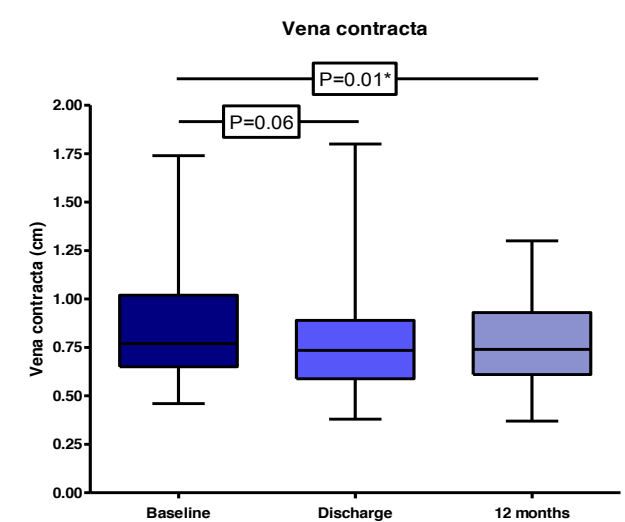

$E$

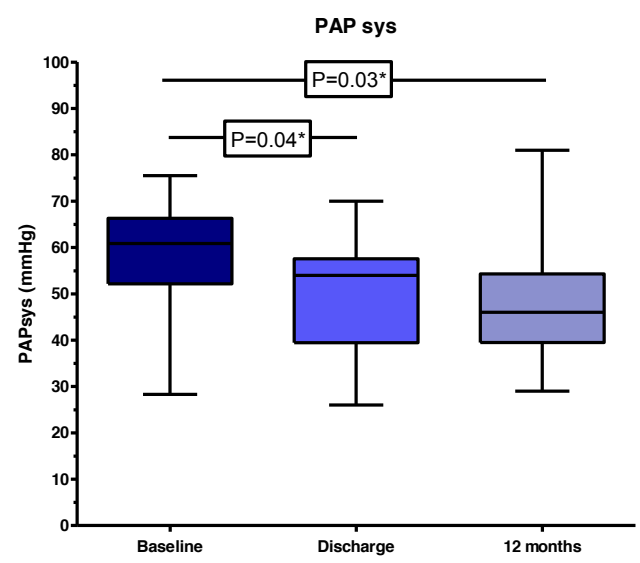

B

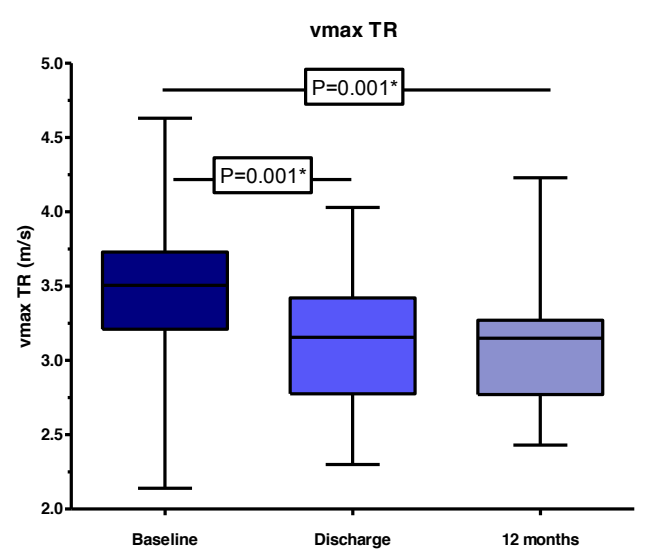

$\mathrm{D}$

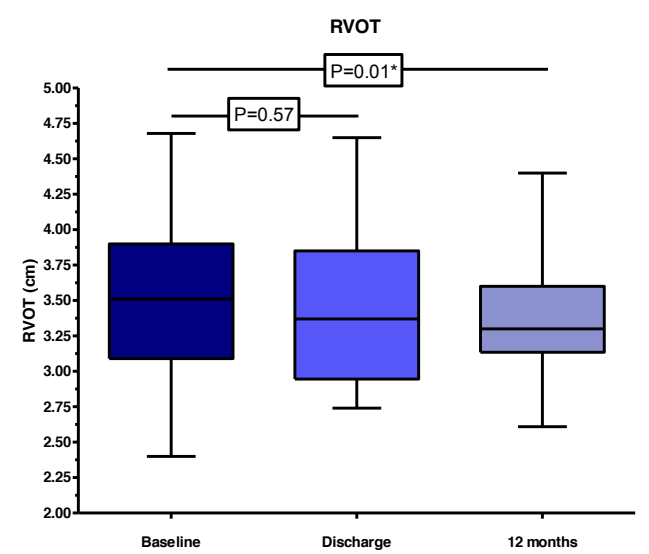

Abbildung 16: Entwicklung rechtsventrikulärer Parameter zum Entlassungszeitpunkt sowie nach 12 Monaten (A: TAPSE; B: maximale Geschwindigkeit des Trikuspidalinsuffizienzjets; C: systolischer pulmonal-arterieller Druck; D: Vena contracta des Trikuspidalklappeninsuffizienzjets; E: Breite des RVOT), gezeigt im box and whiskers-Diagramm: Dargestellt sind jeweils Minimum und Maximum (whiskers), 25. und 75. Perzentile (box) sowie Mediane. 


\subsection{Langzeitüberleben nach MitraClip@-Implantation bei Patienten mit sekundärer Rechtsherzinsuffizienz}

Entsprechend den Einschlusskriterien der Studie lag das Überleben der Patienten im ersten Jahr nach der MitraClip@-Implantation bei $100 \%$. Von diesen Patienten verstarben im zweiten Jahr nach Intervention allerdings $21 \%$, sodass das Überleben der Patientenkohorte innerhalb dieses Jahres auf $79 \%$ sank. Im dritten Jahr nach perkutaner Mitralklappenreparatur sank das Überleben der Gesamtkohorte lediglich um weitere 10 Prozentpunkte und schien im weiteren Verlauf ein relativ stabiles Niveau zu erreichen (vgl. Abbildung 17).

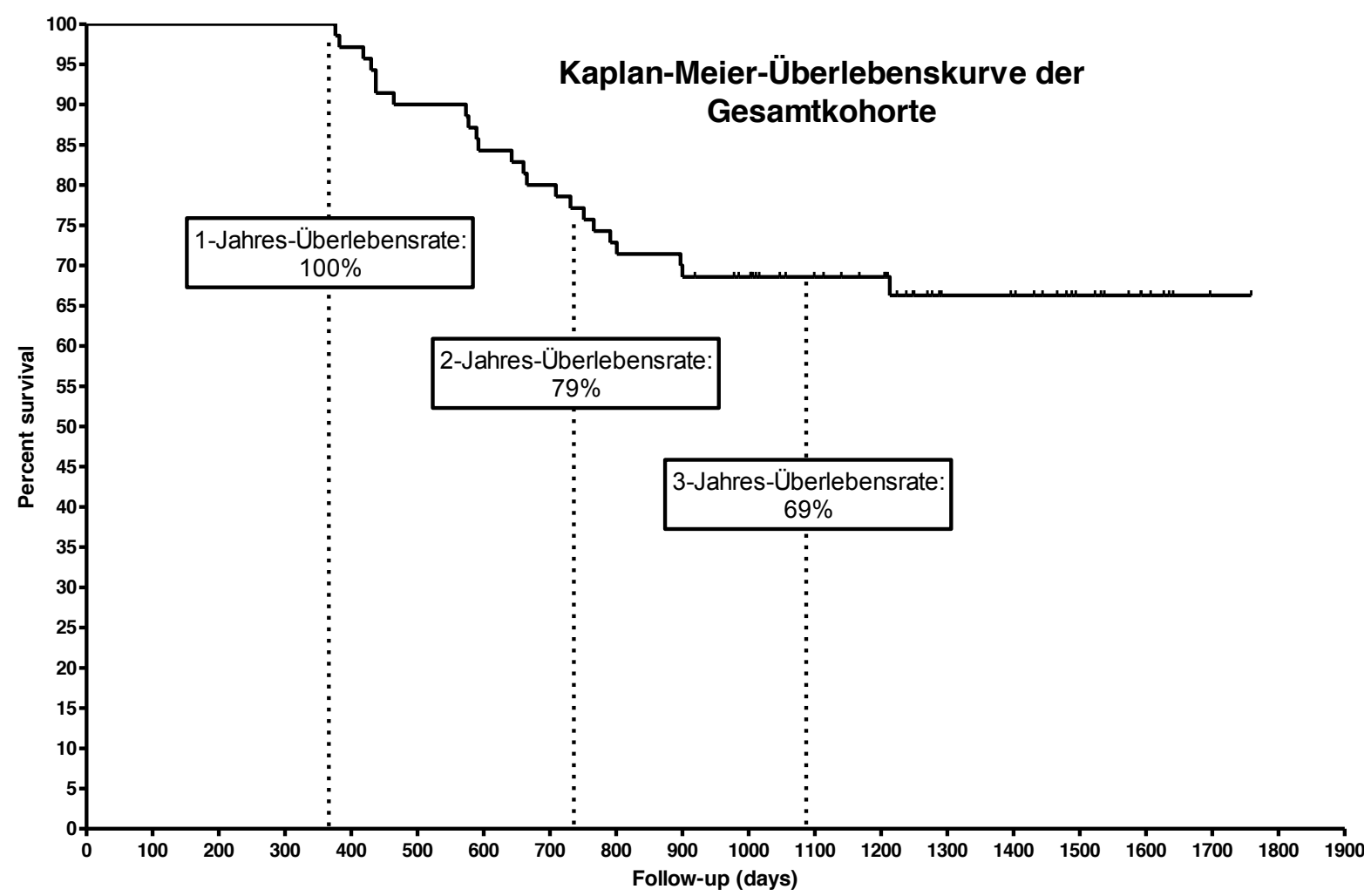

Abbildung 17: Kaplan-Meier-Überlebenskurve der Gesamtkohorte 


\subsection{Langzeitüberleben in Abhängigkeit vom präinterventionellen rechtsventrikulären systolischen Druck}

Im Vergleich des Langzeitüberlebens der Patienten unserer Kohorte in Abhängigkeit vom rechtsventrikulären Druck (abgeschätzt mit Hilfe des maximalen Druckgradienten über der Trikuspidalklappe, $\mathrm{P}$ max über TK) zeigte sich ein tendenziell längeres Überleben bei Patienten mit einem präinterventionellen Pmax unterhalb des Medianwertes von $48,5 \mathrm{mmHg}$ im Vergleich zu Patienten mit einem höheren rechtsventrikulären systolischen Druck $(p=0,13)$. Entsprechend einer Hazard Ratio von 2,09 war die Mortalität der Patienten mit einem rechtsventrikulären systolischen Druck von unter $48,5 \mathrm{mmHg}$ geringer als die der Patienten mit erhöhten präinterventionellen Druckwerten (vgl. Abbildung 18).

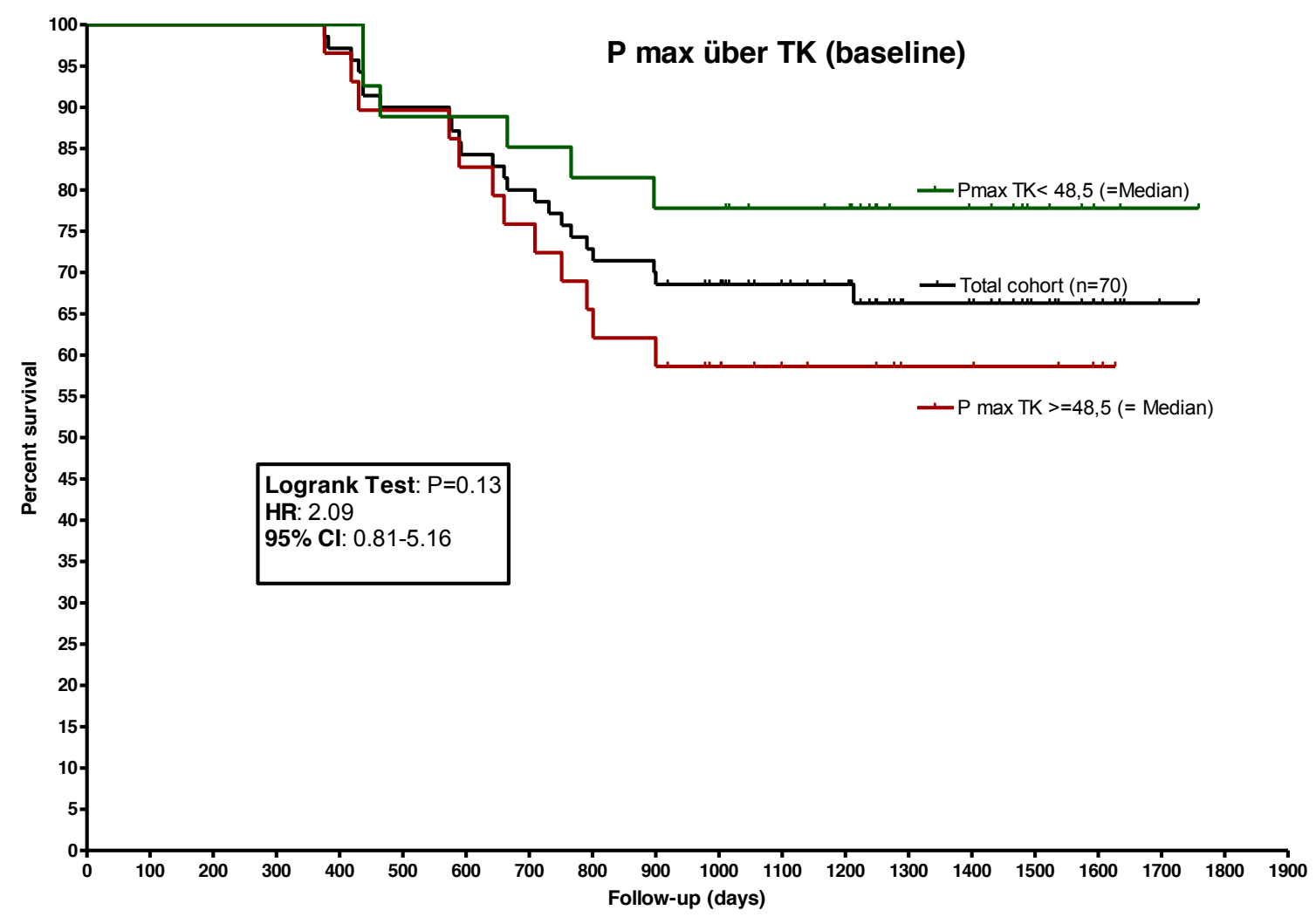

Abbildung 18: Kaplan-Meier-Überlebenskurve in Abhängigkeit vom rechtsventrikulären systolischen Druck 


\subsection{Langzeitüberleben in Abhängigkeit von der präinterventionellen} rechtsventrikulären Kontraktilität (abgeschätzt durch TAPSE) vor dem Eingriff

Die präinterventionelle rechtsventrikuläre Kontraktilität, abgeschätzt durch die TAPSEMessung erwies sich nicht als prädiktiv für das Langzeitüberleben. Es zeigte sich kein signifikanter Überlebensvorteil für Patienten, deren präinterventionelle TAPSE-Werte über dem Median von 16 mm lagen im Vergleich zu Patienten, die einen stärker eingeschränkten TAPSE-Wert vor Intervention aufwiesen ( $p=0,8)$ (vgl. Abbildung 19).

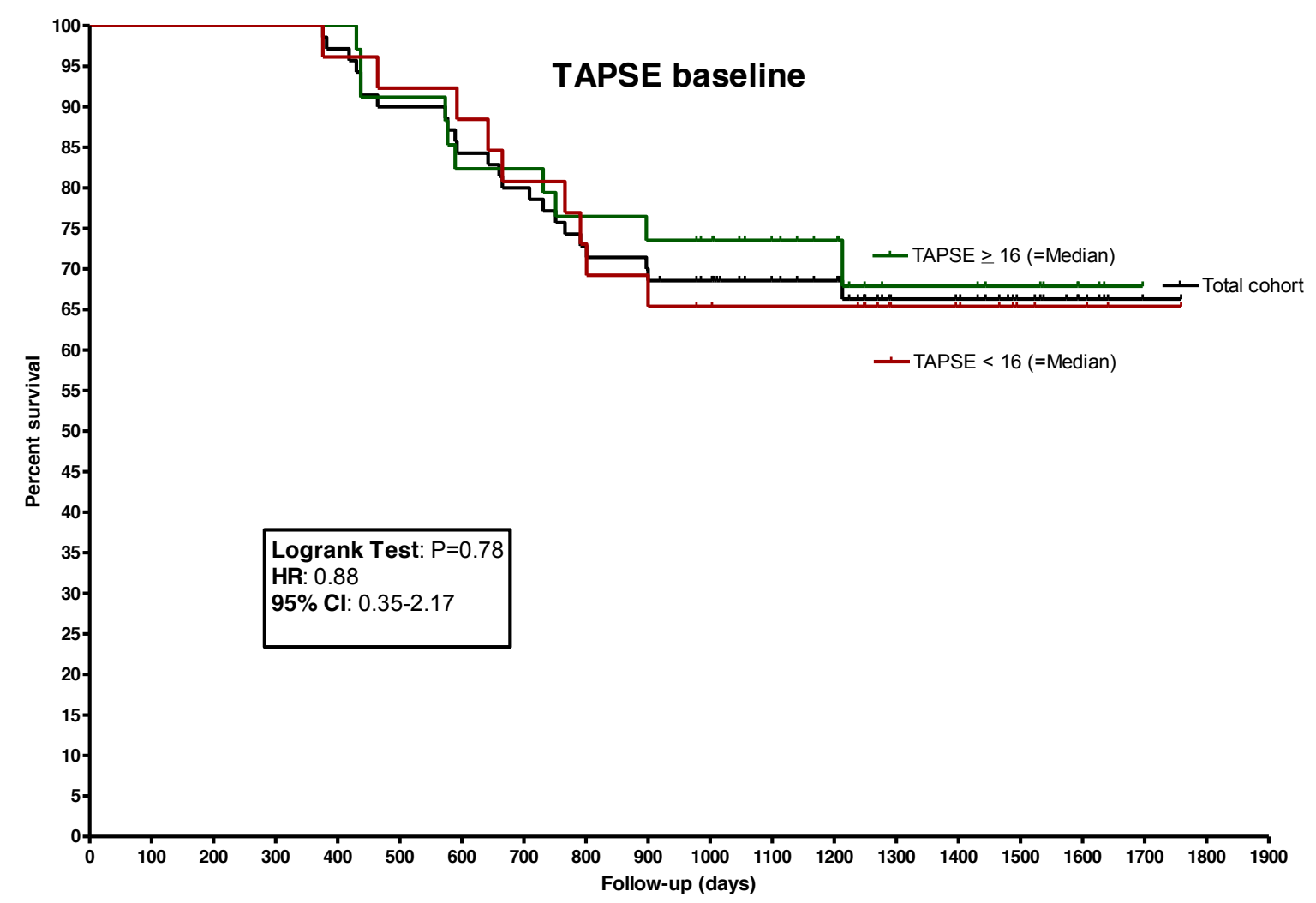

Abbildung 19: Kaplan-Meier-Überlebenskurve in Abhängigkeit von der präinterventionellen rechtsventrikulären Kontraktilität (abgeschätzt durch TAPSE) 


\subsection{Langzeitüberleben in Abhängigkeit von der Veränderung der} rechtsventrikulären Kontraktilität (abgeschätzt durch TAPSE) nach dem Eingriff

Wir untersuchten weiterhin, ob eine Verbesserung der rechtsventrikulären Kontraktilität infolge des Eingriffes zu einem verbesserten Langzeitüberleben führen könnte. Bei 17 Patienten kam es zu einer Normalisierung des TAPSE-Wertes auf $\geq 16 \mathrm{~mm}$, während 33 Patienten sowohl vor als auch nach dem Eingriff einen normalen TAPSE-Wert gezeigt hatten und dieser Wert bei weiteren 15 Patienten < $16 \mathrm{~mm}$ geblieben war oder sich dahin verschlechtert hatte. Es zeigte sich ein signifikanter Zusammenhang der Entwicklung der rechtsventrikulären Funktion mit dem Langzeitüberleben. Die beste Prognose hatten dabei die Patienten, deren TAPSE-Wert sich normalisiert hatte, und die schlechteste diejenigen, deren Wert pathologisch geblieben oder geworden war $(p=0,03)$ (vgl. Abbildung 20).

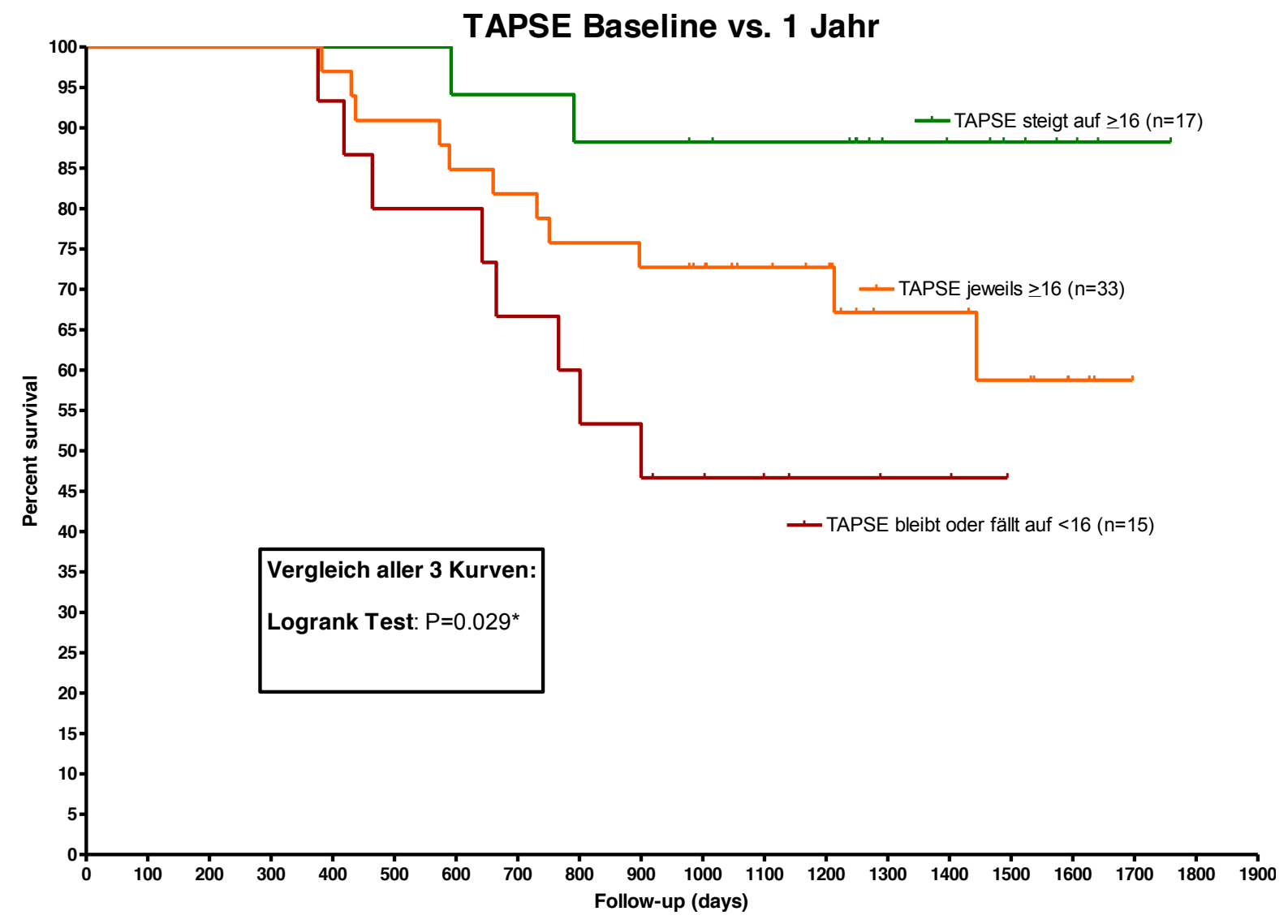

Abbildung 20: Kaplan-Meier-Überlebenskurve in Abhängigkeit von der Veränderung der rechtsventrikulären Kontraktilität (abgeschätzt durch TAPSE) nach dem Eingriff 


\subsection{Langzeitüberleben in Abhängigkeit von der Veränderung des rechtsventrikulären Druckes nach dem Eingriff}

Eine ähnliche Analyse führten wir in Bezug auf die Veränderung des rechtsventrikulären Druckes nach dem Eingriff durch. Hier konnten wir jedoch keinen Unterschied hinsichtlich des Langzeitüberlebens feststellen bei Patienten, deren rechtsventrikulärer Druck infolge des Eingriffes unter $40 \mathrm{mmHg}$ sank, und solchen, bei denen dies nicht der Fall war. Lediglich die Patienten, deren rechtsventrikulärer Druck schon vor dem Eingriff unter $40 \mathrm{mmHg}$ gelegen hatte, zeigten tendenziell ein besseres Überleben (vgl. Abbildung 21).

\section{P max TK Baseline vs. 1 Jahr}

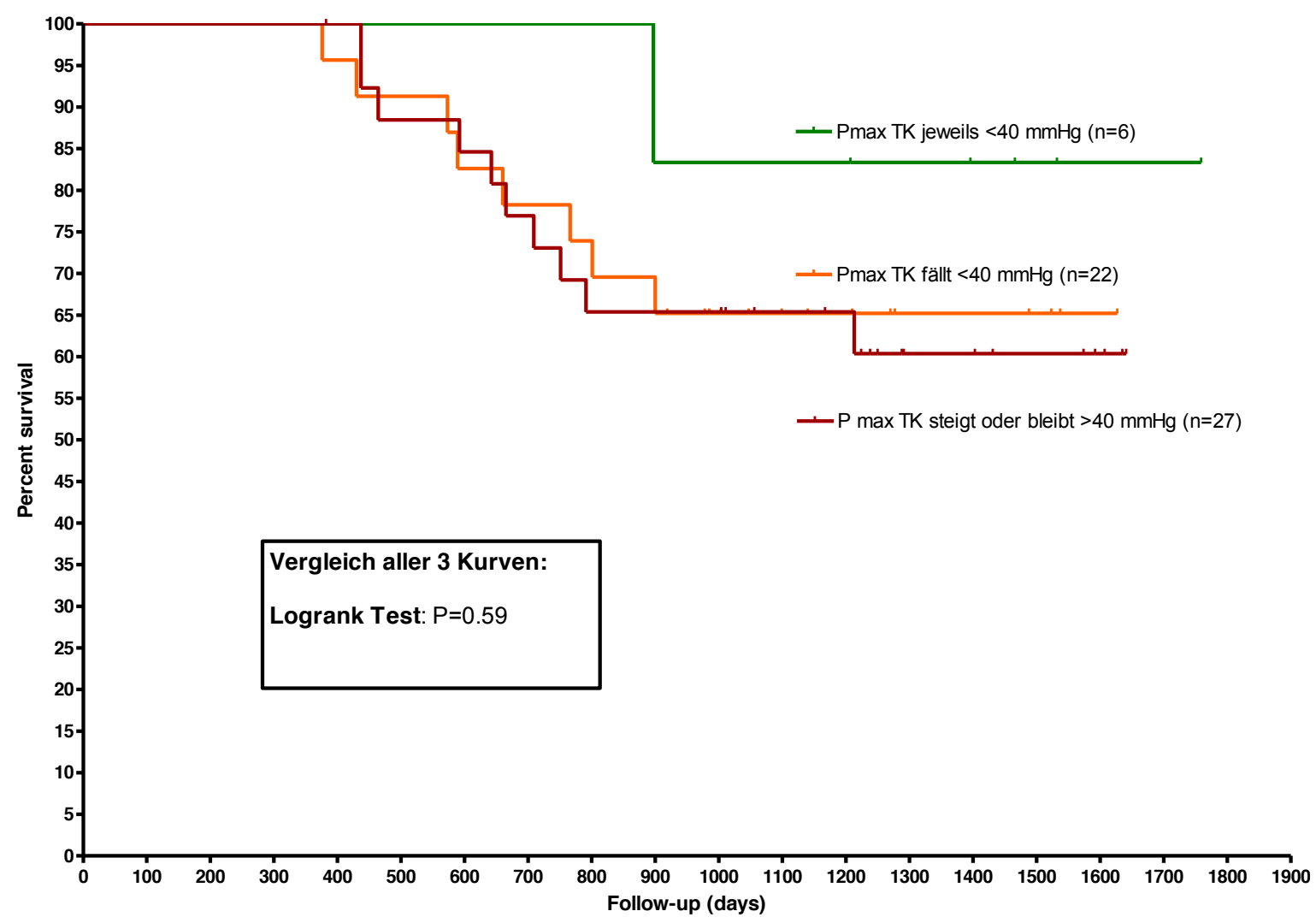

Abbildung 21: Kaplan-Meier-Überlebenskurve in Abhängigkeit von der Veränderung des rechtsventrikulären Druckes nach dem Eingriff 


\section{Diskussion}

Die perkutane Behandlung der Mitralinsuffizienz mittels MitraClip@-Implantation stellt eine neue minimal-invasive Therapiealternative dar. In Deutschland ist diese Therapie nur inoperablen oder chirurgischen Hochrisikopatienten vorbehalten: Demzufolge sind MitraClip@-Patientenkollektive im Allgemeinen gekennzeichnet durch ein hohes Lebensalter und multiple schwerwiegende Begleiterkrankungen. Es ist Gegenstand aktueller Forschung, welche Patienten von diesem Verfahren am meisten und welche möglicherweise nicht mehr profitieren können. Das Vorliegen einer klinischen und/ oder echokardiographischen Rechtsherzinsuffizienz konnte dabei in mehreren Studien als Prädiktor erhöhter Mortalität nach MitraClip@-Implantation identifiziert werden (Neuss et al. 2013; Puls et al. 2014a). Dabei wurde bereits vorgeschlagen, bei einem TAPSE-Wert $<15 \mathrm{~mm}$ als Ausdruck einer Rechtsherzinsuffizienz die Indikation zur Durchführung diese Eingriffes kritisch zu prüfen und eher eine konservative Therapie zu verfolgen (Neuss et al. 2013).

Demgegenüber kann unsere Studie eine signifikante Verbesserung mehrerer Rechtsherzparameter infolge einer MitraClip@-Implantation demonstrieren: Unsere Ergebnisse zeigen bereits unmittelbar nach erfolgreicher MitraClip@-Implantation eine signifikante Reduktion der Flussgeschwindigkeit und folglich des maximalen Druckgradienten über der Trikuspidalklappeninsuffizienz. Auch der systolische pulmonal-arterielle Druck wird somit signifikant gesenkt. Die abfallende Tendenz dieser Werte setzte sich im Nachbeobachtungszeitraum von 12 Monaten fort, ohne jedoch im Vergleich zum Entlassungszeitpunkt erneut signifikant zu werden. Ähnliche Soforteffekte der MitraClip@Implantation auf die hämodynamischen Rechtsherzparameter konnten bereits von einer Schweizer Arbeitsgruppe festgestellt werden (Gaemperli et al. 2012). Mittels Swan-GanzKatheter konnte dort eine statistisch signifikante Reduktion des pulmonal-kapillären Verschlussdruckes (PCWP) von $17 \mathrm{mmHg}$ auf $12 \mathrm{mmHg}(p=0,002)$ sowie eine Reduktion des mittleren pulmonal-arteriellen Druckes von $29 \mathrm{mmHg}$ auf $24 \mathrm{mmHg} \quad(p=0,013)$ nachgewiesen werden. Mit 56\% überwog auch in dieser Patientenkohorte die sekundäre Ätiologie der Mitralinsuffizienz. Eine weitere Nachbeobachtung der Patienten erfolgte allerdings nicht.

Des Weiteren zeigen unsere Ergebnisse unmittelbar nach erfolgreicher Intervention eine signifikante Zunahme der TAPSE als Zeichen einer Verbesserung der rechtsventrikulären Kontraktilität. Auch diese Entwicklung setzte sich in den folgenden 12 Monaten fort, ohne jedoch statistisch erneut signifikant zu werden. Ähnliche Veränderungen der TAPSE infolge einer MitraClip@-Implantation konnten von Giannini et al. beobachtet werden (Giannini et al. 2014). Dort konnte mittels transthorakaler Echokardiographie eine signifikante Verbesserung 
der TAPSE von 16,8 mm auf 18,7 mm ( $p=0,05)$ zum Zeitpunkt der Entlassung festgestellt werden. Obwohl sich diese Entwicklung über den Beobachtungszeitraum von 6 Monaten fortsetzen konnte, wurde auch dort keine erneute Signifikanz erreicht. Mit gleicher Geschlechterverteilung (66\% männlich), vergleichbarem durchschnittlichem Patientenalter (75 Jahre vs. hier 72,5 Jahre), Ätiologie der Mitralinsuffizienz (100\% sekundär vs. $71 \%$ hier) und logistischem EuroSCORE (20\% vs. hier $30 \%$ ) ist das Patientenkollektiv dieser Studie unserem ähnlich. Jedoch ist das Patientenkollektiv mit 35 Patienten lediglich halb so groß. Darüber hinaus haben wir strengere Einschlusskriterien gewählt, da Patienten mit anderen Ursachen als der Mitralinsuffizienz für die sekundäre pulmonale Hypertonie und folglich der Rechtsherzinsuffizienz von unserer Studie ausgeschlossen wurden. Außerdem haben wir mit 12 Monaten einen doppelt so langen Beobachtungszeitraum gewählt, was einem Langzeitverlauf näher kommt.

Die beschriebenen unmittelbaren Veränderungen von pulmonal-arteriellem Druck, Druckgradient, Flussgeschwindigkeit und TAPSE deuten darauf hin, dass sie durch die perkutane Mitralklappenreparatur an sich hervorgerufen wurden. Die minimalinvasive MitraClip@-Implantation bewirkt eine Verringerung des linksventrikulären beziehungsweise linksatrialen Regurgitationsvolumens. Dies führt direkt zu einer reduzierten Volumenbelastung der Lungenstrombahn und damit sekundär zu einer Entlastung des vorgeschalteten rechten Ventrikels, wodurch sich dessen verbesserte Kontraktilität erklären lässt. Wie vorbeschrieben benötigen pulmonale Umbauprozesse oder vaskuläre Adaptationen deutlich mehr Zeit und scheinen an den genannten hämodynamischen Soforteffekten nach MitraClip@-Implantation nicht signifikant beteiligt zu sein. Möglicherweise sind diese Mechanismen aber ursächlich für die Fortsetzung der positiven Entwicklung der Soforteffekte während des weiteren Nachbeobachtungszeitraumes von 12 Monaten.

Diese unmittelbaren positiven hämodynamischen Effekte der MitraClip@-Implantation auf das rechte Herz decken sich nicht mit den Ergebnissen der EVEREST-I-Studie. Dort änderte sich weder systolischer noch diastolischer pulmonal-arterieller Druck signifikant nach Intervention (Siegel et al. 2011). Eine mögliche Erklärung für diese Diskrepanz stellt die bereits erwähnte, unterschiedliche Zusammensetzung des Patientenkollektivs dar. Insbesondere der Unterschied zwischen überwiegend primärer Ätiologie der Mitralinsuffizienz in der EVEREST-I-Kohorte und der überwiegend sekundären Ätiologie der Mitralinsuffizienz in unserem Patientenkollektiv ist hier zu beachten. Darüber hinaus erfolgte die Messung der postinterventionellen hämodynamischen Werte im Rahmen der EVEREST-I-Studie noch während der für die MitraClip@-Implantation notwendigen Vollnarkose. Im Gegensatz dazu wurde die postinterventionelle Echokardiographie bei unseren Patienten in wachem Zustand, 
mindestens 24 Stunden nach dem Eingriff, aber vor Entlassung aus der Klinik durchgeführt. Dieser Unterschied stellt eine weitere mögliche Erklärung für die unterschiedlichen Ergebnisse dar.

Zusätzlich zu den statistisch signifikanten Verbesserungen zum Zeitpunkt der Entlassung

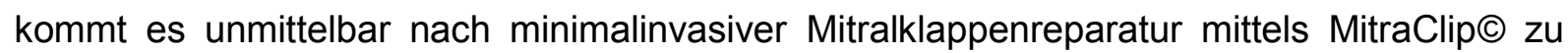
einer Verkleinerung der Vena contracta der Trikuspidalinsuffizienz und einer Abnahme der Breite des rechtsventrikulären Ausflusstraktes (RVOT). Eine statistische Signifikanz dieser Reduktionen beziehungsweise Veränderungen wird allerdings erst nach 12 Monaten erreicht. Aufgrund der Dauer dieser Veränderungen lassen sie sich nicht alleine durch die Reduktion des Regurgitationsvolumens, verbunden mit einer verringerten pulmonalen Volumen- und Druckbelastung, erklären. Für das linke Herz konnten zahlreiche Studien zeigen, dass die minimalinvasive Mitralklappenreparatur einer Mitralinsuffizienz mittels MitraClip@ innerhalb von 6 Monaten zu einem reversen Remodelling des linken Ventrikels und Atriums führt (Franzen et al. 2011; Feldman et al. 2011b; Scandura et al. 2012). Es erscheint logisch, dass für die beschriebenen Veränderungen des rechten Herzens auch Umbauprozesse in der Lungenstrombahn sowie ein reverses Remodelling des rechten Ventrikels an sich ursächlich sind, die bis zu ihrer vollen Ausprägung allerdings mehr Zeit benötigen.

Diese These wird unterstützt durch die Ergebnisse von zwei anderen Arbeitsgruppen. Sowohl Giannini et al. als auch van Riel et al. konnten in einem Nachbeobachtungszeitraum von 6 Monaten keine signifikanten Veränderungen der rechtsventrikulären Parameter nach MitraClip@-Implantation zeigen (Giannini et al. 2014; van Riel et al. 2014). Beide Patientenkollektive sind unserem in den basisdemographischen Charakteristika ähnlich, wobei unsere Studie sowohl in Bezug auf Beobachtungszeitraum als auch Größe der Patientenkohorte und Strenge der Einschlusskriterien einzigartig ist. Eine hämodynamische Entlastung des rechten Ventrikels mit nachfolgendem reversen Remodelling für sechs Monate scheint nicht ausreichend $\mathrm{zu}$ sein, um eine signifikante Reduktion von Vena contracta der Trikuspidalinsuffizienz und des Durchmessers des RVOT zu erreichen.

Im Gegensatz zu den beiden oben genannten Werten konnten wir allerdings keine weiteren rechtsventrikulären Parameter identifizieren, die sich im Nachbeobachtungszeitraum von 12 Monaten nach Intervention signifikant änderten. Auch die Weite des Trikuspidalklappenanulus und der Diameter der Vena cava inferior veränderten sich in unserer Patientenkohorte nicht signifikant. Die Gründe hierfür sind jedoch unklar und bleiben spekulativ. Möglicherweise reicht ein reverses Remodelling des rechten Ventrikels über 12 
Monate nicht aus, um diese Parameter zu beeinflussen. Inwieweit ein verlängerter Nachbeobachtungszeitraum eindeutige Ergebnisse erbringen kann, sollte durch weitere Studien untersucht werden. Um möglichst große Patientenkollektive erreichen zu können, bieten sich dafür multizentrische Studien an.

Insgesamt zeigten sich also bei Patienten mit Mitralinsuffizienz und sekundärer Rechtsherzbelastung statistisch signifikante Veränderungen in der Hämodynamik des rechten Herzens infolge der perkutanen Mitralklappenreparatur mittels MitraClip@. Diese traten zum Teil schon direkt nach Intervention auf oder benötigten 12 Monate, um statistische Signifikanz zu erlangen.

Passend dazu konnten wir anhand der Göttinger Patientenkohorte zeigen, dass die Patienten von der MitraClip@-Implantation klinisch sowohl direkt nach Intervention als auch langfristig deutlich profitierten. Nicht nur fühlten sich die Patienten entsprechend den Ergebnissen des Minnesota Living with Heart Failure Questionnaires durch ihre Herzerkrankung weniger in ihrer Lebensqualität beeinträchtigt, sondern auch ihre Leistungsfähigkeit steigerte sich als Ausdruck einer verlängerten Gehstrecke im SechsMinuten-Gehtest signifikant und nachhaltig. Entsprechend verringerte sich auch die durchschnittliche NYHA-Klassifikation der Patienten bereits nach sechs Monaten signifikant um eine NYHA-Klasse. Angesichts der normalen Progredienz der Mitralinsuffizienz ist insbesondere die Konstanz der Ergebnisse über ein Jahr beachtlich.

Ein weiteres Ziel dieser Studie war die Beantwortung der Frage, ob diese Veränderungen in der Hämodynamik einen Einfluss auf das Überleben der Patienten hatten.

Wie bereits erwähnt, konnten aktuelle Studien klinische und echokardiographische Zeichen einer Rechtsherzbelastung als Prädiktoren einer erhöhten Mortalität nach MitraClip@Implantation identifizieren (Neuss et al. 2013; Puls et al. 2014a). Da in die vorliegende Studie nur Patienten mit klinischer Rechtsherzinsuffizienz eingeschlossen wurden und keine Kontrollgruppe vorlag, konnten wir eine solche Analyse nicht durchführen. Tendenziell zeigte die Hälfte der Patienten, deren systolischer Pulmonalisdruck über dem Median von 48,5 $\mathrm{mmHg}$ lag, ein besseres Überleben $(p=0,13)$, während eine Stratifizierung nach rechtsventrikulärer Kontraktilität mittels TAPSE hier keine relevante Tendenz zeigte. Dabei ist allerdings zu beachten, dass ein Einschlusskriterium unserer Studie das Vorliegen eines 1-Jahres-Follow-Ups darstellte, was alle Patienten ausschloss, die innerhalb des ersten Jahres nach MitraClip-Implantation verstarben. Diese Positiv-Selektion erschwert die Identifikation von Mortalitätsprädiktoren. Auch bei der oben erfolgten Interpretation der klinischen Tests ist diese Selektion zu beachten. 
Dennoch lassen sich Aussagen darüber treffen, wie sich Veränderungen der Hämodynamik auf das Langzeitüberleben der Patienten auswirken. So konnte unsere Studie zeigen, dass Patienten, deren rechtsventrikuläre Kontraktilität sich infolge der MitraClip@-Implantation auf Werte > $16 \mathrm{~mm}$ verbesserte, ein statistisch signifikant längeres Überleben hatten als Patienten mit weiterhin oder neu aufgetretenen eingeschränkten TAPSE-Werten ein Jahr nach Intervention. Dieser Überlebensvorteil konnte bislang von keiner anderen Arbeitsgruppe nachgewiesen werden, hat aus unserer Sicht aber hohe Relevanz für die klinische Praxis. So haben Neuss et al. 2013 einen Algorithmus entwickelt, der alte Patienten mit schwerer Herzinsuffizienz von einer MitraClip@-Implantation ausschließt, sofern die TAPSE präinterventionell kleiner $15 \mathrm{~mm}$ ist (Neuss et al. 2013). Angesichts der in dieser Studie nachgewiesenen möglichen Verbesserung der TAPSE und des damit verbundenen Überlebensvorteils ist dieser Algorithmus kritisch zu betrachten. Zwar haben die Patienten ein verkürztes Überleben im Vergleich mit solchen, die präinterventionell keine Rechtsherzinsuffizienz haben. Gemäß den Ergebnissen unserer Studie profitieren diese Patienten allerdings von der MitraClip@-Implantation. Solange es keine prospektiven Studien gibt, die zeigen, dass dieser Überlebensvorteil auch mit medikamentöser Therapie erreicht werden kann, ist es fragwürdig, den Patienten generell eine MitraClip@-Therapie vorzuenthalten. Es ist vielmehr erforderlich, Faktoren zu identifizieren, mit Hilfe derer bereits präinterventionell abgeschätzt werden kann, welche Patienten mit Rechtsherzinsuffizienz ein rechtsventrikuläres Regenerationspotential aufweisen und welche nicht, um möglichst geeignete Kandidaten für die MitraClip@-Implantation identifizieren zu können, die von dieser Intervention auch klinisch profitieren können. Es ist denkbar, dass die Dauer der Rechtsherzbelastung hier eine Rolle spielen könnte. 


\subsection{Limitationen}

Der Einfluss des Studiendesigns auf die Analyse möglicher Prädiktoren eines verbesserten Langzeitüberlebens von Patienten mit sekundärer Rechtsherzbelastung nach perkutaner Mitralklappenreparatur mittels MitraClip@ $@$ wurde bereits erläutert. Darüber hinaus wäre ein noch größeres Patientenkollektiv wünschenswert gewesen. Dies hätte unter anderem eine erste, grundlegende Identifikation von Faktoren ermöglicht, die eine Verbesserung der rechtsventrikulären Kontraktilität innerhalb des ersten Jahres nach Intervention wahrscheinlich machen.

Unabhängig davon ist zu beachten, dass in diese Studie die jeweils ersten Patienten eingeschlossen wurden, die in den Herzzentren Göttingen und Hamburg mittels MitraClip@ behandelt wurden. Schillinger et al. konnten an den ersten 75 Patienten, die im Herzzentrum Göttingen mittels perkutaner Mitrakalppenreparatur behandelt wurden, einen signifikanten Einfluss der Lernkurve auf die Reduktion der Mitralinsuffizienz durch MitraClip@-Implantation nachweisen (Schillinger et al. 2011). Somit wäre es denkbar, dass eine heute durchgeführt Studie zu noch positiveren Ergebnissen käme.

In Bezug auf die echokardiographische Analyse der rechtsventrikulären Dynamik wäre ein Nachbeobachtungszeitraum von mehr als einem Jahr wünschenswert. Möglicherweise wäre so ein eindeutigerer Nachweis eines reversen rechtsventrikulären Remodellings möglich. Außerdem wurde der rechte Ventrikel in unserer Studie mittels 2D-TTE evaluiert. Aufgrund der komplexen Morphologie des rechten Ventrikels hat sich hierfür die 3D Echokardiographie als überlegen erwiesen (van der Zwaan et al. 2011). Weitere Studien konnten zeigen, dass die systolische Exkursion der Trikuspidalebene (TAPSE) bei hochgradiger Trikuspidalinsuffizienz nur schlecht mit der rechtsventrikulären Pumpfunktion nach Simpson korreliert (Hsiao et al. 2006). Um den pulmonal-arteriellen Druck mittels Echokardiographie messen zu können, war es allerdings Einschlusskriterium in die Studie, dass Patienten eine mittel- bis hochgradige Trikuspidalinsuffizienz aufwiesen.

Eine weitere Limitation dieser Studie besteht darin, dass die in der klinischen Routine durchgeführten TTEs von zwei verschiedenen Untersuchern in Hinblick auf die RechtsherzParameter ausgewertet wurden. Das Einsetzen eines unabhängigen Core-Labs ist in zukünftigen multizentrischen Studien zu empfehlen. 


\subsection{Fazit}

Bei Patienten mit sekundärer Rechtsherzbelastung verbessert die perkutane Mitralklappenreparatur mittels MitraClip@-Verfahren die rechtsventrikuläre Kontraktilität statistisch signifikant und bewirkt eine sofortige und langfristig stabile Reduktion des pulmonal-arteriellen Druckes. Weitere Rechtsherzparameter werden ebenfalls signifikant verbessert, jedoch braucht es weitere multizentrische Studien mit einem längeren Nachbeobachtungszeitraum, um ein vollständiges reverses rechtsventrikuläres Remodelling zu evaluieren.

Patienten, deren rechtsventrikuläre Kontraktilität, abgeschätzt durch TAPSE, sich innerhalb des ersten Jahres nach MitraClip@ auf normale Werte verbessert, haben dabei ein signifikant verlängertes Überleben. Prospektive klinische Studien sind notwendig, um diesen Überlebensvorteil mit der optimalen medikamentösen Therapie bei sekundärer Rechtsherzinsuffizienz zu vergleichen. Bis dahin ist eine eingeschränkte TAPSE als Kriterium gegen eine MitraClip@-Implantation kritisch zu sehen.

Weitere prospektive Studien sind daher notwendig, um bereits präinterventionell abschätzen zu können, welche Patienten mit Rechtsherzinsuffizienz ein rechtsventrikuläres Regenerationspotential aufweisen und welche nicht, um möglichst geeignete Kandidaten für die MitraClip@-Implantation identifizieren zu können. 


\section{Zusammenfassung}

Hintergrund: Die perkutane Mitralklappenreparatur mittels MitraClip@ ist mittlerweile ein etabliertes Verfahren zur Behandlung der Mitralinsuffizienz (MI), in dessen Folge ein reverses linksventrikuläres Remodelling nachgewiesen werden konnte. Die Beschreibung eines reversen rechtsventrikulären Remodellings nach MitraClip@ ist dagegen bisher nicht erfolgt. Zahlreiche Studien konnten pulmonale Hypertonie und Rechtsherzinsuffizienz als Mortalitätsprädiktoren sowohl bei chirurgisch als auch bei minimal-invasiv behandelten Patienten identifizieren. Erste Studien haben eine TAPSE $<15 \mathrm{~mm}$ als Entscheidungskriterium gegen eine MitraClip@-Implantation vorgeschlagen.

Wir untersuchten den Einfluss einer MitraClip@-Implantation auf das rechte Herz bei Patienten mit hochgradiger symptomatischer Mitralklappeninsuffizienz und sekundärer Rechtsherzinsuffizienz. Zusätzlich untersuchten wir den Einfluss der Veränderungen dieser Rechtsherzparameter auf das Überleben der Patienten.

Methoden: $\quad$ Zwischen 2009 und 2011 wurden an den Herzzentren Göttingen und Hamburg 321 Patienten gescreent (Göttingen 84, Hamburg 237). In die Studie eingeschlossen wurden 70 Patienten (Göttingen 31, Hamburg 39), die an einer schweren MI und sekundärer pulmonaler Hypertonie mit klinischen Zeichen der Rechtsherzinsuffizienz litten. Patienten mit anderen Ursachen für die pulmonale Hypertonie wurden aus der Studie ausgeschlossen.

Alle Patienten wurden vor dem Eingriff, vor Klinikentlassung und 12 Monate nach Intervention mittels transthorakaler Echokardiographie untersucht. Im Herbst 2013 wurden alle Patienten telefonisch kontaktiert, um erneute Todesfälle zu erfassen. Kontinuierliche Variablen wurden (in Abwesenheit einer Normalverteilung) mittels Wilcoxon matched pairsTest für gepaarte Stichproben verglichen. Überlebenszeitanalysen wurden mittels KaplanMeier-Kurven visualisiert und mittels eines logrank-Tests verglichen. Dabei wurde jeweils ein p-Wert $<0,05$ als statistisch signifikant gewertet.

Ergebnisse: $\quad$ Das Durchschnittsalter des Gesamtkollektivs betrug $73 \pm 9$ Jahre, $66 \%$ $(n=46)$ waren männlich. Mit $71 \% \quad(n=50)$ überwog die sekundäre Ätiologie der Mitralinsuffizienz. Die Patienten waren klinisch schwer herzinsuffizient (NYHA III $\geq 94 \%$, $n=66$ ) und wiesen ein hohes geschätztes operatives Risiko auf (STS-Score $10 \pm 4 \%$, log. EuroScore I $30 \pm 12 \%$ ).

Wir konnten eine statistisch signifikante Reduktion des pulmonal-arteriellen Druckes unmittelbar nach MitraClip@-Implantation beobachten (60,85 vs. $54,0 \mathrm{mmHg} ; \mathrm{p}=0,04)$. Diese Reduktion blieb nach 12 Monaten stabil $(46,0 \mathrm{mmHg}, \mathrm{p}=0,03)$, ohne im Vergleich zu den 
Messungen zu Klinikentlassung erneute Signifikanz zu gewinnen. Ebenso reduzierte sich die max. Geschwindigkeit der Trikuspidalregurgitation $(3,51$ vs. $3,16 \mathrm{~m} / \mathrm{s}, \mathrm{p}=0,001)$ bereits zum Zeitpunkt der Klinikentlassung statistisch signifikant, blieb nach 12 Monaten stabil $(3,15 \mathrm{~m} / \mathrm{s}$, $p=0,001$ ) und gewann dabei keine erneute Signifikanz. Bereits zum Zeitpunkt der Klinikentlassung beobachteten wir eine signifikante Zunahme der TAPSE (16,0 mm vs. 20,0 $\mathrm{mm}, \mathrm{p}=0,002)$. Diese blieb nach 12 Monaten stabil $(18,9 \mathrm{~mm}, \mathrm{p}=0,001 \mathrm{im}$ Vergleich zum Ausgangswert). Die Diameter der Vena contracta und des rechtsventrikulären Ausflusstraktes waren zum Zeitpunkt der Klinikentlassung noch nicht signifikant reduziert (3,52 vs. $3,44 \mathrm{~cm}, p=0,57$ bzw. 0,77 vs. $0,74 \mathrm{~cm}, p=0,06$ ), konnten diese Signifikanz im Vergleich zu den Baseline-Werten jedoch nach 12 Monaten erreichen $(3,30 \mathrm{~cm}, p=0,01 \mathrm{bzw}$. $0,74 \mathrm{~cm}, \mathrm{p}=0,01)$.

Bei 17 Patienten kam es zu einer Normalisierung des TAPSE- Wertes auf $\geq 16 \mathrm{~mm}$, während 33 Patienten sowohl vor als auch nach dem Eingriff einen normalen TAPSE- Wert gezeigt hatten und dieser Wert bei weiteren 15 Patienten $<16 \mathrm{~mm}$ geblieben war oder sich dahin verschlechtert hatte. Es zeigte sich ein signifikanter Zusammenhang der Entwicklung der rechtsventrikulären Funktion mit dem Langzeitüberleben. Die beste Prognose hatten dabei die Patienten, deren TAPSE- Wert sich normalisiert hatte, und die schlechteste diejenigen, deren Wert pathologisch geblieben oder geworden war $(p=0,03)$.

Zusammenfassung: Die MitraClip@-Implantation bei Patienten mit schwerer Mitralinsuffizienz und sekundärer Rechtsherzinsuffizienz verbessert die Hämodynamik des rechten Herzens akut und langfristig, ohne dabei zu einem vollständigen rechtsventrikulären Remodelling zu führen. Eine Verbesserung der TAPSE auf normale Werte geht mit einem verlängerten Überleben der Patienten einher. Prospektive klinische Studien sind notwendig, um diesen Überlebensvorteil mit der optimalen medikamentösen Therapie bei sekundärer Rechtsherzinsuffizienz zu vergleichen. Bis dahin ist eine eingeschränkte TAPSE als Kriterium gegen eine MitraClip@-Implantation kritisch zu sehen. Außerdem sind weitere Studien notwendig, um Faktoren zu identifizieren, die eine Regenerationsfähigkeit des rechten Herzens begünstigen.

Schlüsselwörter: MitraClip@, perkutane Mitralklappenreparatur, TAPSE, sekundäre Rechsherzbelastung 


\section{Literaturverzeichnis}

Acker MA, Parides MK, Perrault LP, Moskowitz AJ, Gelijns AC, Voisine P, Smith PK, Hung JW, Blackstone EH, Puskas JD, et al. (2014): Mitral-Valve Repair versus Replacement for Severe Ischemic Mitral Regurgitation. N Engl J Med $\underline{370}$, 23-32

Aeschlimann A, Siegenthaler W: Siegenthalers Differenzialdiagnose: innere Krankheiten vom Symptom zur Diagnose ; 323 Tabellen. Thieme, Stuttgart [u.a.] 2005

Alfieri O, De Bonis M, Lapenna E, Regesta T, Maisano F, Torracca L, La Canna G (2004): "Edge-to-edge" repair for anterior mitral leaflet prolapse. Semin Thorac Cardiovasc Surg $\underline{16}, 182-187$

American Thoracic Society (2002): ATS Statement: Guidelines for the Six-Minute Walk Test. Am J Respir Crit Care Med 166, 111-117

Auricchio A, Schillinger W, Meyer S, Maisano F, Hoffmann R, Ussia GP, Pedrazzini GB, van der Heyden J, Fratini S, Klersy C, et al. (2011): Correction of Mitral Regurgitation in Nonresponders to Cardiac Resynchronization Therapy by MitraClip Improves Symptoms and Promotes Reverse Remodeling. J Am Coll Cardiol 토, 2183-2189

Baehner T, Boehm O, Probst C, Poetzsch B, Hoeft A, Baumgarten G, Knuefermann P (2012): Cardiopulmonary bypass in cardiac surgery. Anaesthesist $\underline{61}, 846-856$

Barbieri A, Bursi F, Grigioni F, Tribouilloy C, Avierinos JF, Michelena HI, Rusinaru D, Szymansky C, Russo A, Suri R, et al. (2011): Prognostic and therapeutic implications of pulmonary hypertension complicating degenerative mitral regurgitation due to flail leaflet: A Multicenter Long-term International Study. Eur Heart J $\underline{32}$, 751-759

Boekstegers P, Hausleiter J, Baldus S, von Bardeleben RS, Beucher H, Butter C, Franzen O, Hoffmann R, Ince H, Kuck KH, et al. (2013): Interventionelle Behandlung der Mitralklappeninsuffizienz mit dem MitraClip®-Verfahren: Empfehlungen des Arbeitskreises Interventionelle Mitralklappentherapie der Arbeitsgemeinschaft Interventionelle Kardiologie (AGIK) der Deutschen Gesellschaft für Kardiologie und der Arbeitsgemeinschaft Leitende Kardiologische Krankenhausärzte e. V. (ALKK). Kardiologe $\underline{7}, 91-104$

Bonow RO, Carabello BA, Chatterjee K, de Leon AC, Faxon DP, Freed MD, Gaasch WH, Lytle BW, Nishimura RA, O'Gara PT, et al. (2006): ACC/AHA 2006 Guidelines for the Management of Patients With Valvular Heart Disease: A Report of the American College of Cardiology/American Heart Association Task Force on Practice Guidelines (Writing Committee to Revise the 1998 Guidelines for the Management of Patients With Valvular Heart Disease): Developed in Collaboration With the Society of Cardiovascular Anesthesiologists: Endorsed by the Society for Cardiovascular Angiography and Interventions and the Society of Thoracic Surgeons. Circulation $\underline{114}$, e84-e231

Carabello BA (2013): The Myocardium in Mitral Regurgitation: A Tale of 2 Ventricles. Circulation 127, 1567-1568

Carpentier A (1983): Cardiac valve surgery--the „French correction“. J Thorac Cardiovasc Surg $\underline{86}, 323-337$ 
Chikwe J, Goldstone AB, Passage J, Anyanwu AC, Seeburger J, Castillo JG, Filsoufi F, Mohr FW, Adams DH (2011): A propensity score-adjusted retrospective comparison of early and mid-term results of mitral valve repair versus replacement in octogenarians. Eur Heart J $\underline{32}, 618-626$

Chrustowicz A, Gackowski A, El-Massri N, Sadowski J, Piwowarska W (2010): Preoperative Right Ventricular Function in Patients with Organic Mitral Regurgitation. Echocardiography 27, 282-285

Crawford MH (1986): Acute Mitral Regurgitation. J Intensive Care Med 1, 329-335

Delgado JF, Conde E, Sánchez V, López-Ríos F, Gómez-Sánchez MA, Escribano P, Sotelo T, de la Cámara AG, Cortina J, de la Calzada CS (2005): Pulmonary vascular remodeling in pulmonary hypertension due to chronic heart failure. Eur $\mathrm{J}$ Heart Fail $\underline{7}$, 1011-1016

Di Dedda U, Pelissero G, Agnelli B, De Vincentiis C, Castelvecchio S, Ranucci M (2013): Accuracy, calibration and clinical performance of the new EuroSCORE II risk stratification system. Eur J Cardiothorac Surg $\underline{43}, 27-32$

Enriquez-Sarano M, Akins CW, Vahanian A (2009): Mitral regurgitation. The Lancet $\underline{373}$, 1382-1394

Erbel R, Kahlert P, Plicht B, Konorza T (2009): Mitralklappeninsuffizienz. Herz 34 , 423-425

Erdmann E: Klinische Kardiologie: Krankheiten des Herzens, des Kreislaufs und der herznahen Gefäße. Springer-Verlag Berlin Heidelberg, Berlin, Heidelberg 2011

Feldman T, Wasserman HS, Herrmann HC, Gray W, Block PC, Whitlow P, St. Goar F, Rodriguez L, Silvestry F, Schwartz A, et al. (2005): Percutaneous Mitral Valve Repair Using the Edge-to-Edge Technique: Six-Month Results of the EVEREST Phase I Clinical Trial. J Am Coll Cardiol $\underline{46}$, 2134-2140

Feldman T, Kar S, Rinaldi M, Fail P, Hermiller J, Smalling R, Whitlow PL, Gray W, Low R, Herrmann HC, et al. (2009): Percutaneous Mitral Repair With the MitraClip System: Safety and Midterm Durability in the Initial EVEREST (Endovascular Valve Edge-toEdge REpair Study) Cohort. J Am Coll Cardiol 54, 686-694

Feldman T, Foster E, Glower DD, Kar S, Rinaldi MJ, Fail PS, Smalling RW, Siegel R, Rose GA, Engeron E, et al. (2011a): Percutaneous Repair or Surgery for Mitral Regurgitation. N Engl J Med $\underline{364}, 1395-1406$

Feldman T, Foster E, Glower DD, Kar S, Rinaldi MJ, Fail PS, Smalling RW, Siegel R, Rose GA, Engeron E, et al. (2011b): Percutaneous Repair or Surgery for Mitral Regurgitation. N Engl J Med $\underline{364}, 1395-1406$

Franzen O, van der Heyden J, Baldus S, Schlüter M, Schillinger W, Butter C, Hoffmann R, Corti R, Pedrazzini G, Swaans MJ, et al. (2011): MitraClip® therapy in patients with end-stage systolic heart failure. Eur J Heart Fail $\underline{13}, 569-576$

Gaemperli O, Moccetti M, Surder D, Biaggi P, Hurlimann D, Kretschmar O, Buehler I, Bettex D, Felix C, Luscher TF, et al. (2012): Acute haemodynamic changes after percutaneous mitral valve repair: relation to mid-term outcomes. Heart $\underline{98}, 126-132$

Galie N, Hoeper MM, Humbert M, Torbicki A, Vachiery J-L, Barbera JA, Beghetti M, Corris P, Gaine S, Gibbs JS, et al. (2009): Guidelines for the diagnosis and treatment of pulmonary hypertension: The Task Force for the Diagnosis and Treatment of 
Pulmonary Hypertension of the European Society of Cardiology (ESC) and the European Respiratory Society (ERS), endorsed by the International Society of Heart and Lung Transplantation (ISHLT). Eur Heart J $\underline{30}, 2493-2537$

Gallego-Delgado M, Arribas-Jimenez A, Santos JG, Garcia AM, Gonzalez-Sanchez T, NietoBallestero F, Martin F, Martín-Moreiras J, Martin-Luengo C (2012): Right Ventricular Dysfunction: Prognosis Value in Mitral Valve Surgery. J Am Coll Cardiol 59, E1995

Geissler HJ, Hölzl P, Marohl S, Kuhn-Régnier F, Mehlhorn U, Südkamp M, de Vivie ER (2000): Risk stratification in heart surgery: comparison of six score systems. Eur J Cardiothorac Surg 17, 400-406

Ghio S, Temporelli PL, Klersy C, Simioniuc A, Girardi B, Scelsi L, Rossi A, Cicoira M, Genta FT, Dini FL (2013): Prognostic relevance of a non-invasive evaluation of right ventricular function and pulmonary artery pressure in patients with chronic heart failure. Eur J Heart Fail $\underline{15}$, 408-414

Ghoreishi M, Evans CF, DeFilippi CR, Hobbs G, Young CA, Griffith BP, Gammie JS (2011): Pulmonary hypertension adversely affects short- and long-term survival after mitral valve operation for mitral regurgitation: Implications for timing of surgery. J Thorac Cardiovasc Surg 142, 1439-1452

Giannini C, Petronio AS, De Carlo M, Guarracino F, Conte L, Fiorelli F, Pieroni A, Di Bello V (2014): Integrated reverse left and right ventricular remodelling after MitraClip implantation in functional mitral regurgitation: an echocardiographic study. Eur Heart $\mathrm{J}$ Cardiovasc Imaging $\underline{15}, 95-103$

Glower DD (2012): Surgical Approaches to Mitral Regurgitation. J Am Coll Cardiol $\underline{60}$, 13151322

Goldstone AB, Chikwe J, Pinney SP, Anyanwu AC, Funt SA, Polanco A, Adams DH (2011): Incidence, Epidemiology, and Prognosis of Residual Pulmonary Hypertension After Mitral Valve Repair for Degenerative Mitral Regurgitation. Am J Cardiol 107, 755-760

Grigioni F, Enriquez-Sarano M, Zehr KJ, Bailey KR, Tajik AJ (2001): Ischemic mitral regurgitation: long-term outcome and prognostic implications with quantitative Doppler assessment. Circulation 103, 1759-1764

Hardegree EL, Sachdev A, Fenstad ER, Villarraga HR, Frantz RP, McGoon MD, Oh JK, Ammash NM, Connolly HM, Eidem BW, et al. (2013): Impaired Left Ventricular Mechanics in Pulmonary Arterial Hypertension: Identification of a Cohort at High Risk. Circ Heart Fail $\underline{6}, 748-755$

Hassoun PM, Mouthon L, Barberà JA, Eddahibi S, Flores SC, Grimminger F, Jones PL, Maitland ML, Michelakis ED, Morrell NW, et al. (2009): Inflammation, Growth Factors, and Pulmonary Vascular Remodeling. J Am Coll Cardiol 54, S10-S19

Herold G: Innere Medizin: eine vorlesungsorientierte Darstellung: unter Berücksichtigung des Gegenstandskataloges für die Ärztliche Prüfung: mit ICD 10-Schlüssel im Text und Stichwortverzeichnis. Herold, Köln 2015

Howell NJ, Head SJ, Freemantle N, van der Meulen TA, Senanayake E, Menon A, Kappetein AP, Pagano D (2013): The new EuroSCORE II does not improve prediction of mortality in high-risk patients undergoing cardiac surgery: a collaborative analysis of two European centres. Eur J Cardiothorac Surg 44, 1006-1011 
Hsiao S-H, Lin S-K, Wang W-C, Yang S-H, Gin P-L, Liu C-P (2006): Severe Tricuspid Regurgitation Shows Significant Impact in the Relationship Among Peak Systolic Tricuspid Annular Velocity, Tricuspid Annular Plane Systolic Excursion, and Right Ventricular Ejection Fraction. J Am Soc Echocardiogr 19, 902-910

Huber G, Glaser F (2013): Guidelines Rechtsherz. J Für Kardiologie-Austrian J Cardiol 21, 38-48

lung B (2003): A prospective survey of patients with valvular heart disease in Europe: The Euro Heart Survey on Valvular Heart Disease. Eur Heart J 24, 1231-1243

January CT, Wann LS, Alpert JS, Calkins H, Cigarroa JE, Cleveland JC, Conti JB, Ellinor PT, Ezekowitz MD, Field ME, et al. (2014): 2014 AHA/ACC/HRS Guideline for the Management of Patients With Atrial Fibrillation. J Am Coll Cardiol 64, 1-76

Jilaihawi H, Hussaini A, Kar S (2011): MitraClip: a novel percutaneous approach to mitral valve repair. J Zhejiang Univ Sci B $\underline{12}, 633-637$

Jokinen JJ, Hippeläinen MJ, Pitkänen OA, Hartikainen JEK (2007): Mitral valve replacement versus repair: propensity-adjusted survival and quality-of-life analysis. Ann Thorac Surg $\underline{84}, 451-458$

Kircher BJ, Himelman RB, Schiller NB (1990): Noninvasive estimation of right atrial pressure from the inspiratory collapse of the inferior vena cava. Am J Cardiol $\underline{66}$, 493-496

Kono T, Sabbah HN, Rosman H, Alam M, Jafri S, Goldstein S (1992): Left ventricular shape is the primary determinant of functional mitral regurgitation in heart failure. J Am Coll Cardiol 20, 1594-1598

Le Tourneau T, Richardson M, Juthier F, Modine T, Fayad G, Polge A-S, Ennezat P-V, Bauters C, Vincentelli A, Deklunder G (2010): Echocardiography predictors and prognostic value of pulmonary artery systolic pressure in chronic organic mitral regurgitation. Heart $\underline{96}, 1311-1317$

Le Tourneau T, Deswarte G, Lamblin N, Foucher-Hossein C, Fayad G, Richardson M, Polge A-S, Vannesson C, Topilsky Y, Juthier F, et al. (2013): Right Ventricular Systolic Function in Organic Mitral Regurgitation: Impact of Biventricular Impairment. Circulation 127, 1597-1608

Maisano F (2015): The evolution of MitraClip in the repair of mitral regurgitation. Eur Heart J 36, 1201-1202

Maisano F, Schreuder JJ, Oppizzi M, Fiorani B, Fino C, Alfieri O (2000): The double-orifice technique as a standardized approach to treat mitral regurgitation due to severe myxomatous disease: surgical technique. Eur J Cardiothorac Surg 17, 201-205

Maisano F, Franzen O, Baldus S, Schäfer U, Hausleiter J, Butter C, Ussia GP, Sievert H, Richardt G, Widder JD, et al. (2013): Percutaneous Mitral Valve Interventions in the Real World. J Am Coll Cardiol $\underline{62}, 1052-1061$

Marak CP, Joy PS, Gupta P, Bukovskaya Y, Guddati AK (2013): Diffuse Alveolar Hemorrhage due to Acute Mitral Valve Regurgitation. Case Rep Pulmonol 2013, 1-5

McMurray JJV, Adamopoulos S, Anker SD, Auricchio A, Bohm M, Dickstein K, Falk V, Filippatos G, Fonseca C, Gomez-Sanchez MA, et al. (2012): ESC Guidelines for the diagnosis and treatment of acute and chronic heart failure 2012: The Task Force for the Diagnosis and Treatment of Acute and Chronic Heart Failure 2012 of the 
European Society of Cardiology. Developed in collaboration with the Heart Failure Association (HFA) of the ESC. Eur Heart J $\underline{33}, 1787-1847$

Miller D, Farah MG, Liner A, Fox K, Schluchter M, Hoit BD (2004): The relation between quantitative right ventricular ejection fraction and indices of tricuspid annular motion and myocardial performance. J Am Soc Echocardiogr 17, 443-447

Morrell NW, Adnot S, Archer SL, Dupuis J, Lloyd Jones P, MacLean MR, McMurtry IF, Stenmark KR, Thistlethwaite PA, Weissmann N, et al. (2009): Cellular and Molecular Basis of Pulmonary Arterial Hypertension. J Am Coll Cardiol 54, S20-S31

Murashita T, Okada Y, Kanemitsu H, Fukunaga N, Konishi Y, Nakamura K, Koyama T (2015): The Impact of Preoperative and Postoperative Pulmonary Hypertension on Long-Term Surgical Outcome after Mitral Valve Repair for Degenerative Mitral Regurgitation. Ann Thorac Cardiovasc Surg 21, 53-58

Nagel E, Stuber M, Hess OM (1996): Importance of the right ventricle in valvular heart disease. Eur Heart J 17, 829-836

Neuss M, Schau T, Schoepp M, Seifert M, Hölschermann F, Meyhöfer J, Butter C (2013): Patient selection criteria and midterm clinical outcome for MitraClip therapy in patients with severe mitral regurgitation and severe congestive heart failure. Eur $\mathrm{J}$ Heart Fail $\underline{15}, 786-795$

Nickenig G, Mohr FW, Kelm M, Kuck K-H, Boekstegers P, Hausleiter J, Schillinger W, Brachmann J, Lange R, Reichenspurner H (2013): Konsensus der Deutschen Gesellschaft für Kardiologie - Herz- und Kreislaufforschung - und der Deutschen Gesellschaft für Thorax-, Herz- und Gefäßchirurgie zur Behandlung der Mitralklappeninsuffizienz. Kardiologe $\underline{7}, 76-90$

Nickenig G, Estevez-Loureiro R, Franzen O, Tamburino C, Vanderheyden M, Lüscher TF, Moat N, Price S, Dall'Ara G, Winter R, et al. (2014): Percutaneous Mitral Valve Edgeto-Edge Repair. J Am Coll Cardiol $\underline{64}, 875-884$

Nishimura RA, Otto CM, Bonow RO, Carabello BA, Erwin JP, Guyton RA, O'Gara PT, Ruiz CE, Skubas NJ, Sorajja P, et al. (2014): 2014 AHA/ACC Guideline for the Management of Patients With Valvular Heart Disease: Executive Summary: A Report of the American College of Cardiology/American Heart Association Task Force on Practice Guidelines. Circulation 129, 2440-2492

Nkomo VT, Gardin JM, Skelton TN, Gottdiener JS, Scott CG, Enriquez-Sarano M (2006): Burden of valvular heart diseases: a population-based study. The Lancet $\underline{368}, 1005$ 1011

Paranskaya L, D'Ancona G, Bozdag-Turan I, Akin I, Kische S, Turan GR, Divchev D, Rehders T, Westphal B, Schubert J, et al. (2013): Percutaneous mitral valve repair with the mitraclip ${ }^{\circledR}$ system: Percutaneous Mitral Valve Repair With MitraClip ${ }^{\circledR}$. Catheter Cardiovasc Interv 81, 1224-1231

Puls M, Schillinger W (2016): Kathetergestützte Mitralklappenreparatur mittels MitraClip: Entwicklung, Studienlage und Einsatz im Klinikalltag. Internist $\underline{57}$, 323-33

Puls M, Tichelbäcker T, Bleckmann A, Hünlich M, von der Ehe K, Beuthner BE, Rüter K, Beißbarth T, Seipelt R, Schöndube F, et al. (2014a): Failure of acute procedural success predicts adverse outcome after percutaneous edge-to-edge mitral valve repair with MitraClip. Eurolntervention J Eur Collab Work Group Interv Cardiol Eur Soc Cardiol $\underline{9}$, 1407-1417 
Puls M, Tichelbäcker T, Bleckmann A, Hünlich M, von der Ehe K, Beuthner BE, Rüter K, Beißbarth T, Seipelt R, Schöndube F, et al. (2014b): Failure of acute procedural success predicts adverse outcome after percutaneous edge-to-edge mitral valve repair with MitraClip. Eurolntervention J Eur Collab Work Group Interv Cardiol Eur Soc Cardiol $\underline{9}$, 1407-1417

Puls M, Lubos E, Boekstegers P, von Bardeleben RS, Ouarrak T, Butter C, Zuern CS, Bekeredjian R, Sievert H, Nickenig G, et al. (2016): One-year outcomes and predictors of mortality after MitraClip therapy in contemporary clinical practice: results from the German transcatheter mitral valve interventions registry. Eur Heart $\mathrm{J} \underline{37}$, 703-712

Rudski LG, Lai WW, Afilalo J, Hua L, Handschumacher MD, Chandrasekaran K, Solomon SD, Louie EK, Schiller NB (2010): Guidelines for the Echocardiographic Assessment of the Right Heart in Adults: A Report from the American Society of Echocardiography. J Am Soc Echocardiogr 23, 685-713

Rüter K, Puls $M$, von der Ehe $K$, Tichelbäcker $T$, Sobisiak $B$, Beuthner BE, Hünlich $M$, Schillinger W (2013): Preclosure of femoral vein access site with the suture-mediated Proglide device during MitraClip implantation. J Invasive Cardiol 25, 508-510

Scandura S, Ussia GP, Capranzano P, Caggegi A, Sarkar K, Cammalleri V, Mangiafico S, Chiarandà M, Immè S, Di Pasqua F, et al. (2012): Left Cardiac Chambers Reverse Remodeling after Percutaneous Mitral Valve Repair with the MitraClip System. J Am Soc Echocardiogr 25, 1099-1105

Schillinger W, Athanasiou T, Weicken N, Berg L, Tichelbäcker T, Puls M, Hünlich M, Wachter R, Helms H-J, Seipelt R, et al. (2011): Impact of the learning curve on outcomes after percutaneous mitral valve repair with MitraClip and lessons learned after the first 75 consecutive patients. Eur J Heart Fail 13, 1331-1339

Schillinger W, Hünlich M, Baldus S, Ouarrak T, Boekstegers $P$, Hink U, Butter C, Bekeredjian R, Plicht B, Sievert H, et al. (2013): Acute outcomes after MitraClip ${ }$ therapy in highly aged patients: results from the German TRAnscatheter Mitral valve Interventions (TRAMI) Registry. Eurolntervention $\underline{9}, 84-90$

Schmidt RF, Lang F, Heckmann M: Physiologie des Menschen mit Pathophysiologie ; mit 85 Tabellen; mit herausnehmbarem Repetitorium. Springer-Medizin-Verlag, Heidelberg 2010

Shuhaiber J, Anderson RJ (2007): Meta-analysis of clinical outcomes following surgical mitral valve repair or replacement. Eur J Cardio-Thorac Surg 31, 267-275

Siegel RJ, Biner S, Rafique AM, Rinaldi M, Lim S, Fail P, Hermiller J, Smalling R, Whitlow PL, Herrmann HC, et al. (2011): The Acute Hemodynamic Effects of MitraClip Therapy. J Am Coll Cardiol $\underline{57}, 1658-1665$

Spinale FG, Ishihra K, Zile M, DeFryte G, Crawford FA, Carabello BA (1993): Structural basis for changes in left ventricular function and geometry because of chronic mitral regurgitation and after correction of volume overload. J Thorac Cardiovasc Surg $\underline{106}$, $1147-1157$

Supino PG, Borer JS, Franciosa JA, Preibisz JJ, Hochreiter C, Isom OW, Krieger KH, Girardi LN, Bouraad D, Forur L (2009): Acceptability and Psychometric Properties of the Minnesota Living With Heart Failure Questionnaire Among Patients Undergoing Heart Valve Surgery: Validation and Comparison With SF-36. J Card Fail 15, 267-277 
Suri RM, Schaff HV, Dearani JA, Sundt TM, Daly RC, Mullany CJ, Enriquez-Sarano M, Orszulak TA (2006): Survival advantage and improved durability of mitral repair for leaflet prolapse subsets in the current era. Ann Thorac Surg $\underline{82}, 819-826$

Tenenholtz NA, Hammer PE, Schneider RJ, Vasilyev NV, Howe RD (2011): On the Design of an Interactive, Patient-Specific Surgical Simulator for Mitral Valve Repair. Proc IEEERSJ Int Conf Intell Robots Syst IEEERSJ Int Conf Intell Robots Syst 2011, $1327-1332$

Vahanian A, Alfieri O, Andreotti F, Antunes MJ, Barón-Esquivias G, Baumgartner H, Borger MA, Carrel TP, De Bonis M, Evangelista A, et al. (2012): Guidelines on the management of valvular heart disease (version 2012). Eur Heart J $\underline{33}, 2451-2496$

van der Zwaan HB, Geleijnse ML, McGhie JS, Boersma E, Helbing WA, Meijboom FJ, RoosHesselink JW (2011): Right ventricular quantification in clinical practice: twodimensional vs. three-dimensional echocardiography compared with cardiac magnetic resonance imaging. Eur J Echocardiogr 12, 656-664

van Riel ACMJ, Boerlage-van Dijk K, de Bruin-Bon RHACM, Araki M, Koch KT, Vis MM, Meregalli PG, van den Brink RBA, Piek JJ, Mulder BJM, et al. (2014): Percutaneous Mitral Valve Repair Preserves Right Ventricular Function. J Am Soc Echocardiogr 27, 1098-1106

Wendt D, Osswald BR, Kayser K, Thielmann M, Tossios P, Massoudy P, Kamler M, Jakob H (2009): Society of Thoracic Surgeons Score Is Superior to the EuroSCORE Determining Mortality in High Risk Patients Undergoing Isolated Aortic Valve Replacement. Ann Thorac Surg $\underline{88}, 468-475$

Ye Y, Desai R, Vargas Abello LM, Rajeswaran J, Klein AL, Blackstone EH, Pettersson GB (2014): Effects of right ventricular morphology and function on outcomes of patients with degenerative mitral valve disease. J Thorac Cardiovasc Surg 148, 2012-2020.e8

Zakeri SA, Panayotova R, Borg AN, Miller CA, Schmitt M (2014): Cardiovascular magnetic resonance validation of fractional changes in annulo-apical angles and tricuspid annular plane systolic excursion for rapid assessment of right ventricular systolic function: Rapid Assessment of RV Function by AAs. J Magn Reson Imaging 40, 133 139 


\section{Anhang}

\subsection{Aufnahmebogen}

\section{Aufnahmebogen Göttinger MitraClip - Kohorte}

\begin{tabular}{|l|}
\hline Name \\
\hline Datum der Untersuchung \\
\hline Datum MC \\
\hline StudienNr. \\
\hline TRAMINr. \\
\hline
\end{tabular}

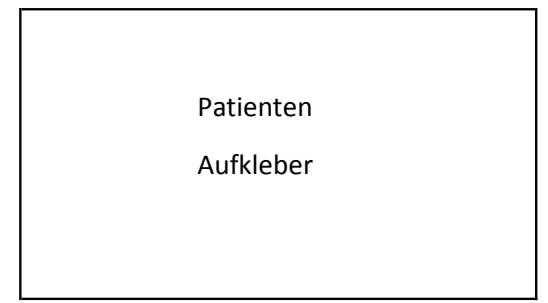

Kontaktdaten

\begin{tabular}{|l|l|}
\hline Telefonnummer & \\
\hline ggf. Angehörige & \\
\hline
\end{tabular}

Krankenhausaufenthale in den letzten 12 Monaten

\begin{tabular}{|l|l|l|}
\hline Datum & Krankenhaus & Aufnahmegrund \\
\hline & & \\
\hline & & \\
\hline & & \\
\hline & & \\
\hline & & \\
\hline
\end{tabular}

NYHA Status

\begin{tabular}{|c|c|c|c|}
\hline I & II & III & IV \\
\hline
\end{tabular}

MLHFQ

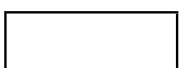

Vitalparameter

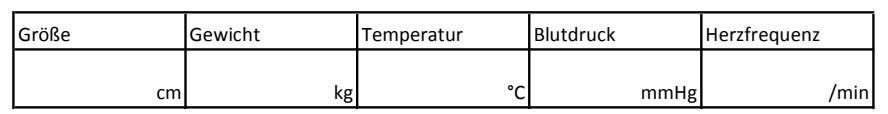

Rauchen in den letzten 12 Monaten?

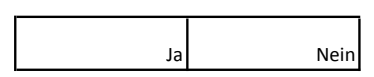

6 Minuten Gehtest

\begin{tabular}{|ll|ll|}
\hline+ & $\mathrm{m}$ & $\mathrm{O}=$ & $\mathrm{m}$ \\
\hline
\end{tabular}

BNP und Trop-T Abnahme organisiert? 


\subsection{Internetmaske EuroSCORE I}

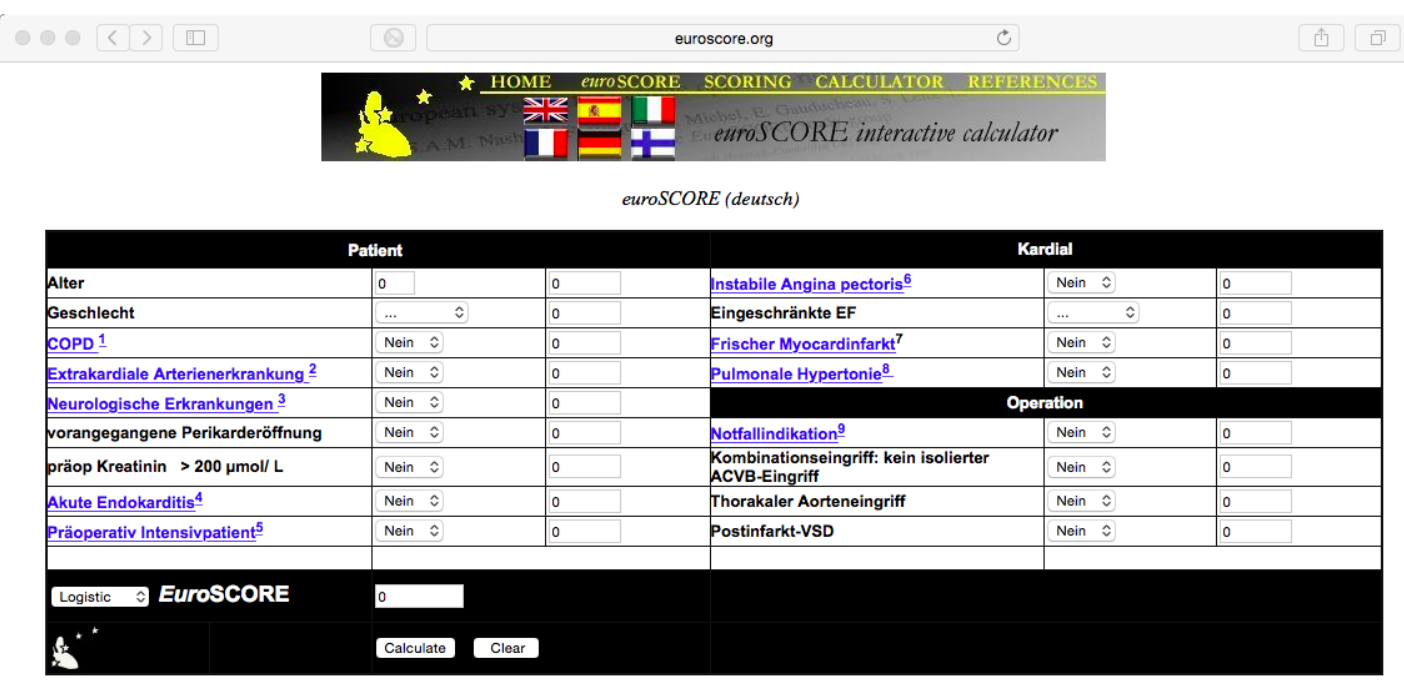

By selecting "Standard euroSCORE" euroSCORE values are simply added to estimate risk of death as described in Roques F, Nashef SA, et al. Eur J Cardiothorac Surg. 1999 Jun;15(6):816By selecting "Logistic euroSCORE" - euroSCORE predicted mortality is calculated as follows (manuscript in preparation):

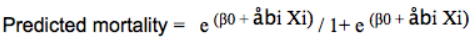

Click here for full details on how to calculate Logistic euroSCORE [Calculator version 1.8 Updated 17th May 2002] Kommentar

[1] COPD Langzeitanwendung von Bronchodilatatoren oder Steroiden

[2] Extrakardiale Arterienerkrankung bei Vorliegen einer der folgenden Symptome/ Erkrankungen: Claudicatio. A. carotis int. Verschluß oder Stenose 50\%. vorausqegangene oder indizierte

\subsection{Internetmaske STS-Score}

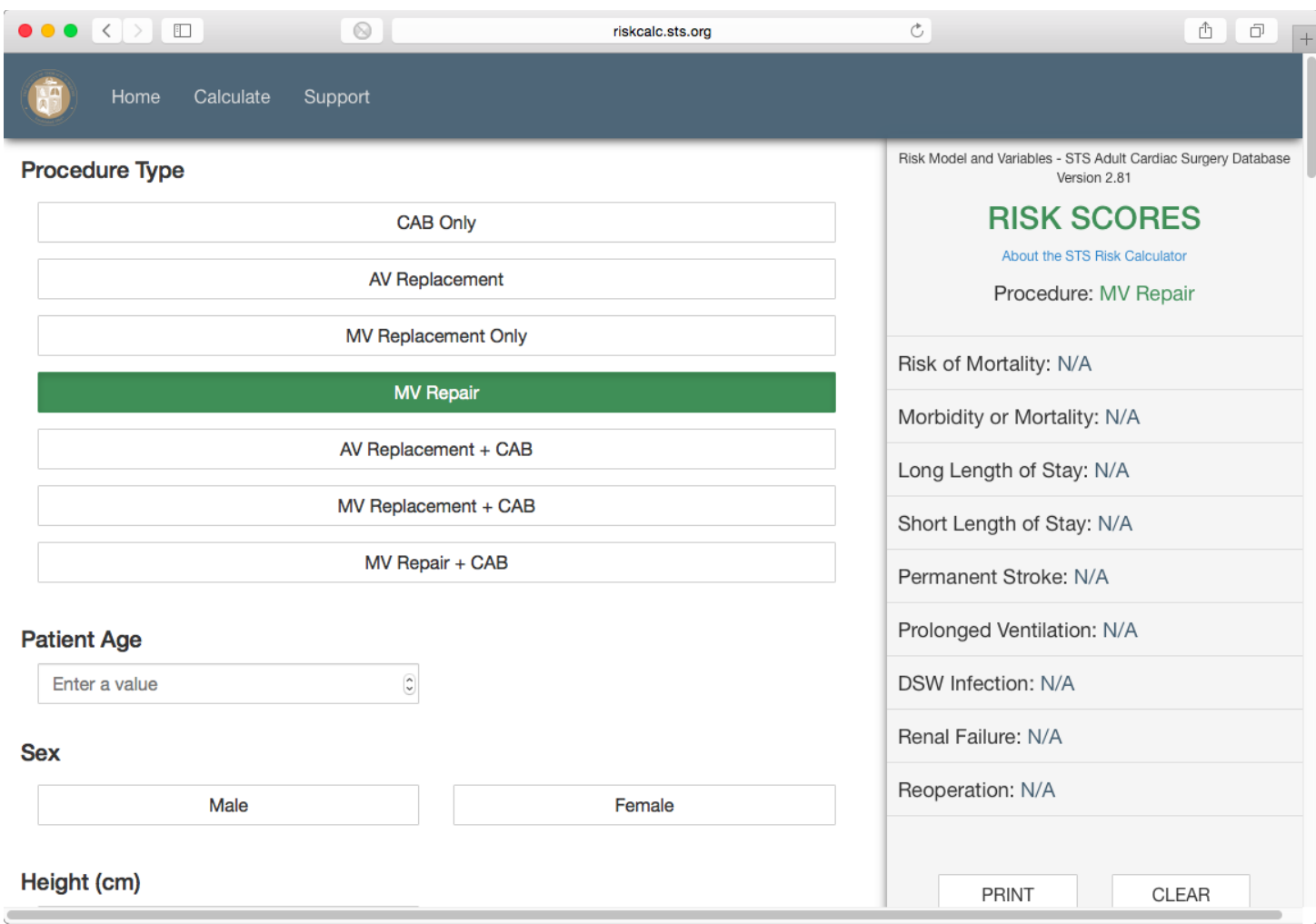




\subsection{Follow-Up Bogen}

\section{FollowUp Untersuchung Göttinger MitraClip Kohorte}

\begin{tabular}{l}
6 Months $\square$ \\
\hline Name \\
\hline Datum MC \\
\hline StudienNr. \\
\hline TRAMINr. \\
\hline ACCESS II Nr. \\
\hline
\end{tabular}

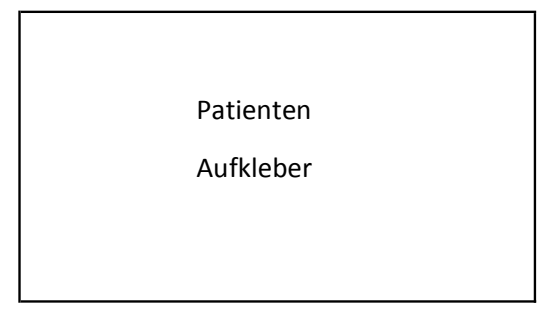

1. Termin des FU

.201

um

Uhr

2. Leitstellenbuch $\square$

Echo/ Miriam $\square$

3. Patient lebt zu Hause

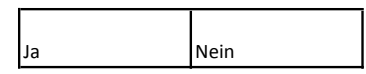

4. Wieviele Tage bis zur Normalen Aktivität

5. NYHA Status

Baseline 6 Months

\begin{tabular}{|l|l|l|l|}
\hline I & II & III & IV \\
\hline
\end{tabular}

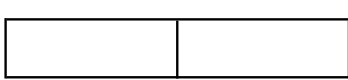

6. Kardiale Krankenhausaufenthalte (seit Clip)

\begin{tabular}{|l|l|l|}
\hline Datum & Krankenhaus & Aufnahmegrund \\
\hline & & \\
\hline & & \\
\hline
\end{tabular}

7. Vitalparameter

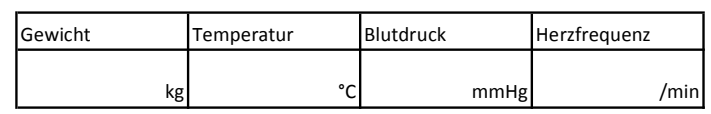

8. 6 Minuten Gehtest

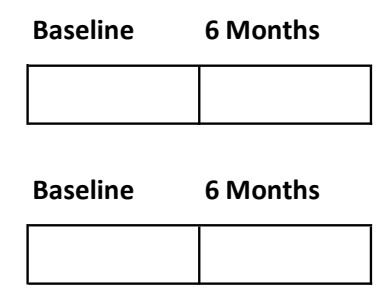

9. MLHFQ 


\subsection{Follow-Up Telefonbogen}

\section{Telefon-Follow-up M itraClip}

\section{Name Patient:}

\section{Datum des Telefongesprächs:}

Mortality:

Patient lebt $\square \quad$ zuhause $\square \quad$ Pflegeheim $\square \quad$ Pflegestufe: keine $\square \quad 1 \square \quad 2 \square \quad 3 \square$ Versorgt sich selbständig ohne Hilfe: Ja $\square \quad$ Nein, Hilfe erforderlich bei:

Patient verstorben $\square \quad$ Datum Ort:

Todesursache:

Entlassungsbrief des letzten stationären Aufenthaltes:

\section{Aktuelle Symptomatik/ Er eignisse:}

Dyspnoe Stadium NYHA

Bei welcher körperlichen Belastung tritt Luftnot auf? (Anzahl Treppenstufen, Meter etc.)

\begin{tabular}{lll}
\hline Ist seit der MitraClip-Implantation ein Schlaganfall aufgetreten? & Ja $\square$ & Nein $\square$ \\
Dialyse seit MitraClip? Ja, seit $\_$bis & Nein $\square$
\end{tabular}

Herzinsuffizienz- oder schlaganfallbedingte Hospitalisationen nach MC (seit letztem FU)

Stat. Aufenthalt 1: Datum Krankenhaus

Ursache Entlassungsbrief

Stat. Aufenthalt 2: Datum Krankenhaus

Ursache Entlassungsbrief

Stat. Aufenthalt 3: Datum Krankenhaus

Ursache Entlassungsbrief

Komplette Daten 6-M onats-Follow-up vor handen:

Falls nein: Vorstellung bei anderem Kardiologen zu diesem Zeitpunkt?

Auswärtiger Befund angefordert/ vorhanden?

Komplette Daten 12-M onats-Follow-up vorhanden:

Falls nein: Vorstellung bei anderem Kardiologen zu diesem Zeitpunkt?

Auswärtiger Befund angefordert/ vorhanden?

$\begin{array}{ll}\text { ja } \square & \text { nein } \square \\ \text { ja } \square & \text { nein } \square \\ \text { ja } \square & \text { nein } \square \\ & \\ \text { ja } \square & \text { nein } \square \\ \text { ja } \square & \text { nein } \square \\ \text { ja } \square & \text { nein } \square\end{array}$

